\title{
A Constrained-Total-Least-Squares Method for Joint Estimation of Source and Sensor Locations: A General Framework
}

\author{
Ding Wang $\mathbb{D},{ }^{1,2}$ Ruirui Liu $\mathbb{D},{ }^{1,2}$ Jiexin Yin, ${ }^{1,2}$ Zhidong $W u,{ }^{1,2}$ \\ Yunlong Wang, ${ }^{1,2}$ and Cheng Wang ${ }^{1,2}$ \\ ${ }^{1}$ National Digital Switching System Engineering and Technology Research Center, Zhengzhou 450002, China \\ ${ }^{2}$ Zhengzhou Information Science and Technology Institute, Zhengzhou, Henan 450002, China \\ Correspondence should be addressed to Ruirui Liu; liu_rr927@163.com
}

Received 14 July 2017; Accepted 8 February 2018; Published 5 April 2018

Academic Editor: Filippo Ubertini

Copyright (C) 2018 Ding Wang et al. This is an open access article distributed under the Creative Commons Attribution License, which permits unrestricted use, distribution, and reproduction in any medium, provided the original work is properly cited.

\begin{abstract}
It is well known that sensor location uncertainties can significantly deteriorate the source positioning accuracy. Therefore, improving the sensor locations is necessary in order to achieve better localization performance. In this paper, a constrained-totalleast-squares (CTLS) method for simultaneously locating multiple disjoint sources and refining the erroneous sensor positions is presented. Unlike conventional localization techniques, an important feature of the proposed method is that it establishes a general framework that is suitable for many different location measurements. First, a modified CTLS optimization problem is formulated after some algebraic manipulations and then the corresponding Newton iterative algorithm is derived to give the numerical solution. Subsequently, by using the first-order perturbation analysis, the explicit expression for the covariance matrix of the proposed CTLS estimator is deduced under the Gaussian assumption. Moreover, the estimation accuracy of the CTLS method is shown to achieve the Cramér-Rao bound (CRB) before the thresholding effect occurs by a rigorous proof. Finally, two kinds of numerical examples are given to corroborate the theoretical development in this paper. One uses the TDOAs/GROAs measurements and the other is based on the TOAs/FOAs parameters.
\end{abstract}

\section{Introduction}

Passive source localization has attracted significant attention in the signal processing research due to its importance to many applications including radar, sonar, microphone arrays, navigation, sensor networks, and wireless communications. Common wireless location systems are based on a twostep procedure for target position determination. In the first phase, the intermediate parameters that depend on the locations of the sources are estimated from the received signals. In general, these parameters include direction of arrival (DOA) [1-3], time of arrival (TOA) [4-7], time difference of arrival (TDOA) [8-17], frequency of arrival (FOA) [18], frequency difference of arrival (FDOA) [19-28], received signal strength (RSS) [29], and gain ratios of arrival (GROA) [30, 31]. In the second phase, the previously estimated parameters are used to locate the sources. During the past few decades, numerous methods have been proposed for the two active research areas. In this paper, we focus on the latter, that is, emitter location estimation.

It is easy to see that the position determination is equivalent to solving a set of nonlinear equations relating the intermediate parameters to the coordinates of the sources. A number of localization methods are available in the literature. Some of them are iterative algorithms (such as Taylor series (TS) algorithm [2, 12, 20, 23, 32] and constrained-total-leastsquares (CTLS) algorithm [3, 6, 15, 17, 25-27]) that require proper initial solution guesses, and the others are closed-form solutions (such as total least squares (TLS) solution [1, 11, 22], quadratic constraint least square (QCLS) solution $[4,9,10$, 13,33 , and two-step weighted least square (TWLS) solution $[5,7,8,14,16,19,21,24,28-31])$ that are more computationally efficient. Most of these algorithms can reach the corresponding Cramér-Rao bound (CRB) accuracy under moderate level of signal-to-noise ratio (SNR). Moreover, it is worth noting that all the localization algorithms need to transform 
the nonlinear measurement equations into pseudo-linear equations, except for the TS algorithm.

However, it must be emphasized that the accuracy of source location estimate may be seriously degraded by the sensor location errors, regardless of the specific localization algorithm used. In [21, 34], the source location mean square error (MSE) is derived when the sensor locations are assumed correct but in fact have errors. Generally, there exist two classes of methods that can mitigate the effects of the uncertainties in sensor location. The first class of methods is to incorporate the statistical characteristic of sensor location errors into the position estimation procedure $[5,6,17,21,24$, $26,27]$, and the second one performs joint estimation of the unknown source locations and the inaccurate sensor positions together $[2,7,12,14,16,20,23,28,31]$. In this work, we concentrate on the latter because it can increase the accuracy of the sensor position estimates and tolerate higher noise level before the thresholding effect caused by nonlinear estimation starts to occur.

It is well known that the TLS technique is an improved least squares (LS) method to solve an overdetermined set of linear equations $\mathbf{A x} \approx \mathbf{y}$ when there are errors not only in the observations $\mathbf{y}$ but in the coefficient matrix $\mathbf{A}$ as well. In $[1,11,22]$, the TLS method is applied to source localization. Note that the TLS solution can be found simply via the singular value decomposition (SVD) technique [35], and therefore, it is computationally attractive. However, the TLS estimator is generally not asymptotically efficient because it assumes that the noise components in $\mathbf{A}$ and $\mathbf{y}$ are independent and identically distributed (i.i.d.), which is rarely realistic in practical scenario. The CTLS method, as a natural extension of the TLS method, is able to fully exploit the noise structure in $\mathbf{A}$ and $\mathbf{y}$ [36], and hence, the resulting solution is shown to achieve the Cramér-Rao bound (CRB). Indeed, the CTLS method has been successfully applied to wireless location. In [3], the CTLS algorithm is proposed to solve the bearing-only localization problem. In [15], the CTLS localization algorithm using TDOA measurements is presented. Additionally, an efficient CTLS algorithm for determining the position and velocity of a moving source based on TDOA and FDOA measurements is developed in [25]. However, it is noteworthy that none of these CTLS algorithms consider the sensor position errors, which may seriously deteriorate the positioning accuracy. In order to reduce the effects of the uncertainties in sensor positions, the robust CTLS localization algorithms are presented in $[6,26$, 27].

While the above-mentioned CTLS algorithms can achieve satisfactory performance, it is necessary to point out that all of them apply only to the single-source scenario, and moreover none of them can provide the joint estimation of source and sensor locations. Furthermore, all the algorithm derivations and theoretical analysis are performed only for some specific measurements, thus leading to the lack of a united framework for this problem. This paper presents an efficient CTLS method that can locate multiple disjoint sources and refine the erroneous sensor positions simultaneously. Different from the existing approaches, the proposed method is derived in a more general framework that is applicable to many different location measurements. First, a modified CTLS optimization problem is formulated after some algebraic manipulations and then the corresponding Newton iterative algorithm is derived to yield the numerical solution. Subsequently, by exploiting the first-order perturbation analysis, the closed-form expression for the covariance matrix of the new CTLS estimator is deduced under the Gaussian assumption. Moreover, the estimation accuracy of the CTLS solution is rigorously proved to reach the CRB before the thresholding effect occurs. Finally, we give two examples to illustrate how to utilize the proposed CTLS method for source localization. One uses the TDOAs/GROAs measurements and the other is based on the TOAs/FOAs parameters. The experiment results support the theoretical development in this paper.

The remainder of this paper is organized as follows. In Section 2, the measurement model for source localization is described and the problem under investigation is formulated. Section 3 derives the modified CTLS optimization model. In Section 4, the Newton iterative algorithm is derived to provide the joint estimation of source and sensor locations. Section 5 provides the closed-form expression for the covariance matrix of the new CTLS estimator and proves its asymptotical efficiency. In Section 6, two examples are given to illustrate how to utilize the proposed CTLS method for source localization. Numerical simulations are presented in Section 7 to support the theoretical development in this paper. Conclusions are drawn in Section 8. The proofs of the main results are shown in the Appendixes (available here).

\section{Measurement Model and Problem Formulation}

2.1. Nonlinear Measurement Model. We consider the localization scenario where $D$ disjoint sources are to be located. Under ideal condition, the location observation equation associated with the $d$ th source can be represented in a generic form as

$$
\mathbf{z}_{d 0}=\mathbf{f}\left(\mathbf{u}_{d}, \mathbf{w}\right) \quad(1 \leq d \leq D),
$$

where $\mathbf{z}_{d 0} \in \mathbf{R}^{p_{1} \times 1}$ is the true measurement vector, $\mathbf{u}_{d} \epsilon$ $\mathbf{R}^{p_{2} \times 1}$ is the position and/or velocity vector of the $d$ th source, $\mathbf{w} \in \mathbf{R}^{p_{3} \times 1}$ denotes the system parameter which contains the sensor positions and/or velocities, and $\mathbf{f}(\cdot, \cdot)$ is the nonlinear function that depends on the specific measurement type used.

Note that if the vectors $\mathbf{z}_{d 0}$ and $\mathbf{w}$ can be accurately obtained, the localization problem is equivalent to solving a set of nonlinear equations. However, $\mathbf{z}_{d 0}$ and $\mathbf{w}$ are not known exactly in practice. First, only the noisy version of $\mathbf{z}_{d 0}$, denoted as $\mathbf{z}_{d}$, is available. It can be written as

$$
\mathbf{z}_{d}=\mathbf{z}_{d 0}+\mathbf{n}_{d}=\mathbf{f}\left(\mathbf{u}_{d}, \mathbf{w}\right)+\mathbf{n}_{d} \quad(1 \leq d \leq D),
$$

where $\mathbf{n}_{d}$ is the measurement noise vector that follows zeromean Gaussian distribution with covariance matrix $\mathbf{N}_{d}=$ $E\left[\mathbf{n}_{d} \mathbf{n}_{d}^{T}\right]$. In addition, the known system parameter, denoted as $\mathbf{v}$, is also erroneous. It can be modeled as

$$
\mathbf{v}=\mathbf{w}+\mathbf{m},
$$


where $\mathbf{m}$ is the noise vector and it is Gaussian distributed with zero-mean and covariance matrix $\mathbf{M}=E\left[\mathbf{m m}^{T}\right]$. Besides, $\mathbf{m}$ and $\left\{\mathbf{n}_{d}\right\}_{1 \leq d \leq D}$ are statistically independent.

2.2. Pseudo-Linear Measurement Model. For some special measurements (e.g., DOA, TOA, TDOA, and GROA), (1) can be transformed into the following pseudo-linear model:

$$
\begin{array}{r}
\mathbf{a}\left(\mathbf{z}_{d 0}, \mathbf{w}\right)=\mathbf{B}\left(\mathbf{z}_{d 0}, \mathbf{w}\right) \mathbf{t}_{d}=\mathbf{B}\left(\mathbf{z}_{d 0}, \mathbf{w}\right) \mathbf{h}\left(\mathbf{u}_{d}, \mathbf{w}\right) \\
(1 \leq d \leq D),
\end{array}
$$

where $\mathbf{a}\left(\mathbf{z}_{d 0}, \mathbf{w}\right) \in \mathbf{R}^{p_{1} \times 1}$ is the pseudo-linear measurement vector and $\mathbf{B}\left(\mathbf{z}_{d 0}, \mathbf{w}\right) \in \mathbf{R}^{p_{1} \times\left(p_{2}+p_{4}\right)}$ is the coefficient matrix, $\mathbf{t}_{d}=\mathbf{h}\left(\mathbf{u}_{d}, \mathbf{w}\right) \in \mathbf{R}^{\left(p_{2}+p_{4}\right) \times 1}$. Vector function $\mathbf{h}\left(\mathbf{u}_{d}, \mathbf{w}\right)$ is given by

$$
\mathbf{h}\left(\mathbf{u}_{d}, \mathbf{w}\right)=\left[\frac{\mathbf{u}_{d}-\mathbf{J w}}{\mathbf{s}\left(\mathbf{u}_{d}, \mathbf{w}\right)}\right],
$$

where $\mathbf{J} \in \mathbf{R}^{p_{2} \times p_{3}}$ is a known and constant matrix and $\mathbf{s}\left(\mathbf{u}_{d}\right.$, $\mathbf{w}) \in \mathbf{R}^{p_{4} \times 1}$ comprises all the instrumental variables whose number is defined by $p_{4}$.

Since every equation in (4) is related to the system parameter $\mathbf{w}$, we must combine these equations to perform joint estimation of all the position vectors $\left\{\mathbf{u}_{d}\right\}_{1<d<D}$ and the system parameter $\mathbf{w}$. In this treatment, we can obtain cooperation gain compared to the approaches which locate the sources individually.

Putting all the $D$ equations in (4) together yields

$$
\widetilde{\mathbf{a}}\left(\widetilde{\mathbf{z}}_{0}, \mathbf{w}\right)=\widetilde{\mathbf{B}}\left(\widetilde{\mathbf{z}}_{0}, \mathbf{w}\right) \widetilde{\mathbf{t}}=\widetilde{\mathbf{B}}\left(\widetilde{\mathbf{z}}_{0}, \mathbf{w}\right) \widetilde{\mathbf{h}}(\widetilde{\mathbf{u}}, \mathbf{w}),
$$

where

$$
\begin{aligned}
& \widetilde{\mathbf{a}}\left(\widetilde{\mathbf{z}}_{0}, \mathbf{w}\right)
\end{aligned}
$$

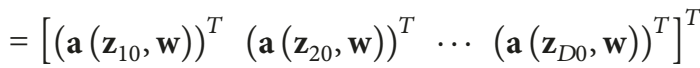

$$
\begin{aligned}
& \in \mathbf{R}^{p_{1} D \times 1} \\
& \widetilde{\mathbf{B}}\left(\widetilde{\mathbf{z}}_{0}, \mathbf{w}\right) \\
& =\operatorname{blkdiag}\left[\mathbf{B}\left(\mathbf{z}_{10}, \mathbf{w}\right) \quad \mathbf{B}\left(\mathbf{z}_{20}, \mathbf{w}\right) \cdots \mathbf{B}\left(\mathbf{z}_{D 0}, \mathbf{w}\right)\right] \\
& \in \mathbf{R}^{p_{1} D \times\left(p_{2}+p_{4}\right) D} \\
& \widetilde{\mathbf{t}}=\tilde{\mathbf{h}}(\widetilde{\mathbf{u}}, \mathbf{w})=\left[\begin{array}{llll}
\mathbf{t}_{1}^{T} & \mathbf{t}_{2}^{T} & \cdots & \mathbf{t}_{D}^{T}
\end{array}\right]^{T} \\
& =\left[\begin{array}{llll}
\left(\mathbf{h}\left(\mathbf{u}_{1}, \mathbf{w}\right)\right)^{T} & \left(\mathbf{h}\left(\mathbf{u}_{2}, \mathbf{w}\right)\right)^{T} & \ldots & \left(\mathbf{h}\left(\mathbf{u}_{D}, \mathbf{w}\right)\right)^{T}
\end{array}\right]^{T} \\
& \in \mathbf{R}^{\left(p_{2}+p_{4}\right) D \times 1} \\
& \widetilde{\mathbf{z}}_{0}=\left[\begin{array}{llll}
\mathbf{z}_{10}^{T} & \mathbf{z}_{20}^{T} & \cdots & \mathbf{z}_{D 0}^{T}
\end{array}\right]^{T} \in \mathbf{R}^{p_{1} D \times 1}, \\
& \widetilde{\mathbf{u}}=\left[\begin{array}{llll}
\mathbf{u}_{1}^{T} & \mathbf{u}_{2}^{T} & \cdots & \mathbf{u}_{D}^{T}
\end{array}\right]^{T} \in \mathbf{R}^{p_{2} D \times 1} .
\end{aligned}
$$

It is obvious from (7) that vector $\widetilde{\mathbf{u}}$ contains the location vectors of all the emitters. In addition, it can also been seen from (7) that vector $\widetilde{\mathbf{z}}_{0}$ comprises the measurement vectors of all the sources. The noisy version of $\widetilde{\mathbf{z}}_{0}$ is denoted by $\widetilde{\mathbf{z}}$, which can be expressed as

$$
\begin{aligned}
\widetilde{\mathbf{z}}= & \widetilde{\mathbf{z}}_{0}+\widetilde{\mathbf{n}} \\
= & {\left[\begin{array}{lll}
\left(\mathbf{f}\left(\mathbf{u}_{1}, \mathbf{w}\right)\right)^{T} \quad\left(\mathbf{f}\left(\mathbf{u}_{2}, \mathbf{w}\right)\right)^{T} & \ldots\left(\mathbf{f}\left(\mathbf{u}_{D}, \mathbf{w}\right)\right)^{T}
\end{array}\right]^{T} } \\
& +\widetilde{\mathbf{n}}=\widetilde{\mathbf{f}}(\widetilde{\mathbf{u}}, \mathbf{w})+\widetilde{\mathbf{n}},
\end{aligned}
$$

where

$$
\begin{aligned}
\widetilde{\mathbf{n}} & =\left[\begin{array}{llll}
\mathbf{n}_{1}^{T} & \mathbf{n}_{2}^{T} & \cdots & \mathbf{n}_{D}^{T}
\end{array}\right]^{T} \in \mathbf{R}^{p_{1} D \times 1} \\
\widetilde{\mathbf{f}}(\widetilde{\mathbf{u}}, \mathbf{w}) & \\
& =\left[\begin{array}{lll}
\left(\mathbf{f}\left(\mathbf{u}_{1}, \mathbf{w}\right)\right)^{T} & \left(\mathbf{f}\left(\mathbf{u}_{2}, \mathbf{w}\right)\right)^{T} \cdots\left(\mathbf{f}\left(\mathbf{u}_{D}, \mathbf{w}\right)\right)^{T}
\end{array}\right]^{T} \\
& \in \mathbf{R}^{p_{1} D \times 1} .
\end{aligned}
$$

It can be readily seen from (9) that the noise vector $\widetilde{\mathbf{n}}$ follows zero-mean Gaussian distribution. Its covariance matrix is defined by $\widetilde{\mathbf{N}}=E\left[\widetilde{\mathbf{n}} \widetilde{\mathbf{n}}^{T}\right]$. If $\mathbf{n}_{d_{1}}$ and $\mathbf{n}_{d_{2}}$ are statistically independent for $d_{1} \neq d_{2}$, then we have $\widetilde{\mathbf{N}}=$ blkdiag $\left[\begin{array}{llll}\mathbf{N}_{1} & \mathbf{N}_{2} & \cdots & \mathbf{N}_{D}\end{array}\right]$.

The positioning problem here can be briefly stated as follows: Given the observation vectors $\left\{\mathbf{z}_{d}\right\}_{1 \leq d \leq D}$ and available system parameter $\mathbf{v}$, find an estimate of $\left\{\mathbf{u}_{d}\right\}_{1 \leq d \leq D}$ (or $\widetilde{\mathbf{u}}$ ) and $\mathbf{w}$ as accurate as possible based on the pseudo-linear equation (6).

\section{Optimization Model}

In (6), the functional forms of $\widetilde{\mathbf{a}}(\cdot, \cdot)$ and $\widetilde{\mathbf{B}}(\cdot, \cdot)$ are known, but vectors $\widetilde{\mathbf{z}}_{0}$ and $\mathbf{w}$ are not available and only their noisy values (i.e., $\widetilde{\mathbf{z}}$ and $\mathbf{v}$ ) can be obtained. In order to establish the CTLS optimization model, we shall perform a first-order Taylor series expansion of $\widetilde{\mathbf{a}}\left(\widetilde{\mathbf{z}}_{0}, \mathbf{w}\right)$ and $\widetilde{\mathbf{B}}\left(\widetilde{\mathbf{z}}_{0}, \mathbf{w}\right)$ around $\widetilde{\mathbf{z}}$ as well as $\mathbf{v}$. It can be verified that

$$
\begin{aligned}
& \widetilde{\mathbf{a}}\left(\widetilde{\mathbf{z}}_{0}, \mathbf{w}\right) \approx \widetilde{\mathbf{a}}(\widetilde{\mathbf{z}}, \mathbf{v})-\mathbf{A}_{1}(\widetilde{\mathbf{z}}, \mathbf{v}) \widetilde{\mathbf{n}}-\mathbf{A}_{2}(\widetilde{\mathbf{z}}, \mathbf{v}) \mathbf{m}, \\
& \widetilde{\mathbf{B}}\left(\widetilde{\mathbf{z}}_{0}, \mathbf{w}\right) \approx \widetilde{\mathbf{B}}(\widetilde{\mathbf{z}}, \mathbf{v})-\sum_{j=1}^{p_{1} D}\langle\widetilde{\mathbf{n}}\rangle_{j} \cdot \dot{\widetilde{\mathbf{B}}}_{1 j}(\widetilde{\mathbf{z}}, \mathbf{v})-\sum_{j=1}^{p_{3}}\langle\mathbf{m}\rangle_{j} \\
& \dot{\widetilde{\mathbf{B}}}_{2 j}(\widetilde{\mathbf{z}}, \mathbf{v}),
\end{aligned}
$$

where

$$
\begin{aligned}
& \widetilde{\mathbf{A}_{1}}(\widetilde{\mathbf{z}}, \mathbf{v})=\frac{\partial \widetilde{\mathbf{a}}(\widetilde{\mathbf{z}}, \mathbf{v})}{\partial \widetilde{\mathbf{z}}^{T}} \in \mathbf{R}^{p_{1} D \times p_{1} D}, \\
& \widetilde{\mathbf{A}_{2}}(\widetilde{\mathbf{z}}, \mathbf{v})=\frac{\partial \widetilde{\mathbf{a}}(\widetilde{\mathbf{z}}, \mathbf{v})}{\partial \mathbf{v}^{T}} \in \mathbf{R}^{p_{1} D \times p_{3}} \\
& \dot{\widetilde{\mathbf{B}}}_{1 j}(\widetilde{\mathbf{z}}, \mathbf{v})=\frac{\partial \widetilde{\mathbf{B}}(\widetilde{\mathbf{z}}, \mathbf{v})}{\partial\langle\widetilde{\mathbf{z}}\rangle_{j}} \in \mathbf{R}^{p_{1} D \times\left(p_{2}+p_{4}\right) D} \quad\left(1 \leq j \leq p_{1} D\right) \\
& \dot{\widetilde{\mathbf{B}}}_{2 j}(\widetilde{\mathbf{z}}, \mathbf{v})=\frac{\partial \widetilde{\mathbf{B}}(\widetilde{\mathbf{z}}, \mathbf{v})}{\partial\langle\mathbf{v}\rangle_{j}} \in \mathbf{R}^{p_{1} D \times\left(p_{2}+p_{4}\right) D} \quad\left(1 \leq j \leq p_{3}\right) .
\end{aligned}
$$


Inserting (10) into (6) leads to

$$
\begin{aligned}
& \widetilde{\mathbf{a}}(\widetilde{\mathbf{z}}, \mathbf{v})-\mathbf{A}_{1}(\widetilde{\mathbf{z}}, \mathbf{v}) \widetilde{\mathbf{n}}-\mathbf{A}_{2}(\widetilde{\mathbf{z}}, \mathbf{v}) \mathbf{m} \\
& \approx \widetilde{\mathbf{B}}(\widetilde{\mathbf{z}}, \mathbf{v}) \widetilde{\mathbf{t}}-\sum_{j=1}^{p_{1} D}\langle\widetilde{\mathbf{n}}\rangle_{j} \cdot \dot{\widetilde{\mathbf{B}}}_{1 j}(\widetilde{\mathbf{z}}, \mathbf{v}) \widetilde{\mathbf{t}} \\
& \quad-\sum_{j=1}^{p_{3}}\langle\mathbf{m}\rangle_{j} \cdot \dot{\widetilde{\mathbf{B}}}_{2 j}(\widetilde{\mathbf{z}}, \mathbf{v}) \widetilde{\mathbf{t}} \Longrightarrow \\
& \widetilde{\mathbf{a}}(\widetilde{\mathbf{z}}, \mathbf{v})-\widetilde{\mathbf{B}}(\widetilde{\mathbf{z}}, \mathbf{v}) \widetilde{\mathbf{t}} \approx \widetilde{\mathbf{C}}_{1}(\widetilde{\mathbf{t}}, \widetilde{\mathbf{z}}, \mathbf{v}) \widetilde{\mathbf{n}}+\widetilde{\mathbf{C}}_{2}(\widetilde{\mathbf{t}}, \widetilde{\mathbf{z}}, \mathbf{v}) \mathbf{m},
\end{aligned}
$$

where

$$
\begin{aligned}
& \widetilde{\mathbf{C}}_{1}(\widetilde{\mathbf{t}}, \widetilde{\mathbf{z}}, \mathbf{v}) \\
&=\widetilde{\mathbf{A}}_{1}(\widetilde{\mathbf{z}}, \mathbf{v}) \\
& \quad-\left[\dot{\widetilde{\mathbf{B}}}_{11}(\widetilde{\mathbf{z}}, \mathbf{v}) \widetilde{\mathbf{t}} \dot{\tilde{\mathbf{B}}}_{12}(\widetilde{\mathbf{z}}, \mathbf{v}) \widetilde{\mathbf{t}} \cdots \dot{\widetilde{\mathbf{B}}}_{1, p_{1} D}(\widetilde{\mathbf{z}}, \mathbf{v}) \widetilde{\mathbf{t}}\right] \\
& \in \mathbf{R}^{p_{1} D \times p_{1} D,} \\
& \widetilde{\mathbf{C}}_{2}(\widetilde{\mathbf{t}}, \widetilde{\mathbf{z}}, \mathbf{v}) \\
&=\widetilde{\mathbf{A}_{2}}(\widetilde{\mathbf{z}}, \mathbf{v}) \\
& \quad-\left[\dot{\widetilde{\mathbf{B}}}_{21}(\widetilde{\mathbf{z}}, \mathbf{v}) \widetilde{\mathbf{t}} \dot{\tilde{\mathbf{B}}}_{22}(\widetilde{\mathbf{z}}, \mathbf{v}) \widetilde{\mathbf{t}} \cdots \dot{\widetilde{\mathbf{B}}}_{2 p_{3}}(\widetilde{\mathbf{z}}, \mathbf{v}) \widetilde{\mathbf{t}}\right] \\
& \in \mathbf{R}^{p_{1} D \times p_{3}} .
\end{aligned}
$$

Note that the problem addressed herein is the joint estimation of $\widetilde{\mathbf{u}}$ and $\mathbf{w}$. Therefore, it is necessary to define an augmented parameter vector as below:

$$
\overline{\widetilde{\mathbf{t}}}=\overline{\widetilde{\mathbf{h}}}(\widetilde{\mathbf{u}}, \mathbf{w})=\left[\begin{array}{c}
\tilde{\mathbf{t}} \\
\mathbf{w}
\end{array}\right]=\left[\begin{array}{c}
\widetilde{\mathbf{h}}(\widetilde{\mathbf{u}}, \mathbf{w}) \\
\mathbf{w}
\end{array}\right] \in \mathbf{R}^{\left(\left(p_{2}+p_{4}\right) D+p_{3}\right) \times 1}
$$

Then, by combining (3) and (12), we can get the following programming model:

$$
\begin{aligned}
\min _{\widetilde{\mathbf{u}}, \mathbf{w}, \widetilde{\mathbf{n}}, \mathbf{m}} & {\left[\begin{array}{l}
\widetilde{\mathbf{n}} \\
\mathbf{m}
\end{array}\right]^{T} \cdot\left[\begin{array}{cc}
\widetilde{\mathbf{N}}^{-1} & \mathbf{O}_{p_{1} D \times p_{3}} \\
\mathbf{O}_{p_{3} \times p_{1} D} & \mathbf{M}^{-1}
\end{array}\right] \cdot\left[\begin{array}{l}
\widetilde{\mathbf{n}} \\
\mathbf{m}
\end{array}\right] } \\
\text { s.t. } & {\left[\begin{array}{c}
\widetilde{\mathbf{a}}(\widetilde{\mathbf{z}}, \mathbf{v}) \\
\mathbf{v}
\end{array}\right]-\left[\begin{array}{cc}
\widetilde{\mathbf{B}}(\widetilde{\mathbf{z}}, \mathbf{v}) & \mathbf{O}_{p_{1} D \times p_{3}} \\
\mathbf{O}_{p_{3} \times\left(p_{2}+p_{4}\right) D} & \mathbf{I}_{p_{3}}
\end{array}\right] \cdot \overline{\mathbf{t}} } \\
& =\left[\begin{array}{cc}
\widetilde{\mathbf{C}}_{1}(\widetilde{\mathbf{t}}, \widetilde{\mathbf{z}}, \mathbf{v}) & \widetilde{\mathbf{C}}_{2}(\widetilde{\mathbf{t}}, \widetilde{\mathbf{z}}, \mathbf{v}) \\
\mathbf{O}_{p_{3} \times p_{1} D} & \mathbf{I}_{p_{3}}
\end{array}\right] \cdot\left[\begin{array}{l}
\widetilde{\mathbf{n}} \\
\mathbf{m}
\end{array}\right] .
\end{aligned}
$$

Although (15) has equality constraint, it can be converted into an unconstrained minimization problem over $\widetilde{\mathbf{u}}$ and $\mathbf{w}$. The details can be found in the following proposition.

Proposition 1. If $\widetilde{\mathbf{C}}_{1}(\widetilde{\mathbf{t}}, \widetilde{\mathbf{z}}, \mathbf{v})$ is an invertible matrix, then the constrained optimization problem (15) can be recast as an equivalent unconstrained one, which is expressed as

$$
\min _{\widetilde{\mathbf{u}}, \mathbf{w}} J_{\mathrm{ctls}}(\widetilde{\mathbf{u}}, \mathbf{w})=\min _{\widetilde{\mathbf{u}}, \mathbf{w}}(\overline{\widetilde{\mathbf{a}}}(\widetilde{\mathbf{z}}, \mathbf{v})-\overline{\widetilde{\mathbf{B}}}(\widetilde{\mathbf{z}}, \mathbf{v}) \overline{\widetilde{\mathbf{t}}})^{T}(\overline{\widetilde{\mathbf{Q}}}(\widetilde{\mathbf{t}}, \widetilde{\mathbf{z}}, \mathbf{v}))^{-1}(\overline{\widetilde{\mathbf{a}}}(\widetilde{\mathbf{z}}, \mathbf{v})-\overline{\widetilde{\mathbf{B}}}(\widetilde{\mathbf{z}}, \mathbf{v}) \overline{\widetilde{\mathbf{t}}}),
$$

where

$$
\begin{aligned}
\overline{\widetilde{\mathbf{z}}}(\widetilde{\mathbf{z}}, \mathbf{v}) & =\left[\begin{array}{c}
\widetilde{\mathbf{a}}(\widetilde{\mathbf{z}}, \mathbf{v}) \\
\mathbf{v}
\end{array}\right] \in \mathbf{R}^{\left(p_{1} D+p_{3}\right) \times 1}, \\
\overline{\widetilde{\mathbf{B}}}(\widetilde{\mathbf{z}}, \mathbf{v}) & =\left[\begin{array}{cc}
\widetilde{\mathbf{B}}(\widetilde{\mathbf{z}}, \mathbf{v}) & \mathbf{O}_{p_{1} D \times p_{3}} \\
\mathbf{O}_{p_{3} \times\left(p_{2}+p_{4}\right) D} & \mathbf{I}_{p_{3}}
\end{array}\right] \in \mathbf{R}^{\left(p_{1} D+p_{3}\right) \times\left(\left(p_{2}+p_{4}\right) D+p_{3}\right)}, \\
\overline{\widetilde{\mathbf{Q}}}(\widetilde{\mathbf{t}}, \widetilde{\mathbf{z}}, \mathbf{v}) & =\left[\begin{array}{c|c}
\widetilde{\mathbf{C}}_{1}(\widetilde{\mathbf{t}}, \widetilde{\mathbf{z}}, \mathbf{v}) \widetilde{\mathbf{N}}\left(\widetilde{\mathbf{C}}_{1}(\widetilde{\mathbf{t}}, \widetilde{\mathbf{z}}, \mathbf{v})\right)^{T}+\widetilde{\mathbf{C}}_{2}(\widetilde{\mathbf{t}}, \widetilde{\mathbf{z}}, \mathbf{v}) \mathbf{M}\left(\widetilde{\mathbf{C}_{2}}(\widetilde{\mathbf{t}}, \widetilde{\mathbf{z}}, \mathbf{v})\right)^{T} & \widetilde{\mathbf{C}}_{2}(\widetilde{\mathbf{t}}, \widetilde{\mathbf{z}}, \mathbf{v}) \mathbf{M} \\
\hline \mathbf{M}\left(\widetilde{\mathbf{C}_{2}}(\widetilde{\mathbf{t}}, \widetilde{\mathbf{z}}, \mathbf{v})\right)^{T} & \mathbf{M}
\end{array}\right] \in \mathbf{R}^{\left(p_{1} D+p_{3}\right) \times\left(p_{1} D+p_{3}\right)} .
\end{aligned}
$$

Proof. Define $\widetilde{\mathbf{n}}^{\prime}=\widetilde{\mathbf{N}}^{-1 / 2} \widetilde{\mathbf{n}}$ and $\mathbf{m}^{\prime}=\mathbf{M}^{-1 / 2} \mathbf{m}$, and then (15) is equivalent to

$$
\min _{\widetilde{\mathbf{u}}, \mathbf{w}, \widetilde{\mathbf{n}}^{\prime}, \mathbf{m}^{\prime}}\left\|\left[\begin{array}{c}
\widetilde{\mathbf{n}}^{\prime} \\
\mathbf{m}^{\prime}
\end{array}\right]\right\|_{2}^{2}
$$

$$
\begin{array}{ll}
\text { s.t. } & {\left[\begin{array}{c}
\widetilde{\mathbf{a}}(\widetilde{\mathbf{z}}, \mathbf{v}) \\
\mathbf{v}
\end{array}\right]-\left[\begin{array}{cc}
\widetilde{\mathbf{B}}(\widetilde{\mathbf{z}}, \mathbf{v}) & \mathbf{O}_{p_{1} D \times p_{3}} \\
\mathbf{O}_{p_{3} \times\left(p_{2}+p_{4}\right) D} & \mathbf{I}_{p_{3}}
\end{array}\right] \cdot \overline{\tilde{\mathbf{t}}}} \\
= & {\left[\begin{array}{cc}
\widetilde{\mathbf{C}}_{1}(\widetilde{\mathbf{t}}, \widetilde{\mathbf{z}}, \mathbf{v}) \widetilde{\mathbf{N}}^{1 / 2} & \widetilde{\mathbf{C}}_{2}(\widetilde{\mathbf{t}}, \widetilde{\mathbf{z}}, \mathbf{v}) \mathbf{M}^{1 / 2} \\
\mathbf{O}_{p_{3} \times p_{1} D} & \mathbf{M}^{1 / 2}
\end{array}\right]}
\end{array}
$$




$$
\cdot\left[\begin{array}{c}
\widetilde{\mathbf{n}}^{\prime} \\
\mathbf{m}^{\prime}
\end{array}\right]
$$

The optimal solution to (19) is given by

$$
\begin{aligned}
& {\left[\begin{array}{c}
\widetilde{\mathbf{n}}^{\prime} \\
\mathbf{m}^{\prime}
\end{array}\right]_{\text {opt }} } \\
&=\left[\begin{array}{cc}
\widetilde{\mathbf{C}}_{1}(\widetilde{\mathbf{t}}, \widetilde{\mathbf{z}}, \mathbf{v}) \\
\widetilde{\mathbf{N}}_{p_{3} \times p_{1} D}^{1 / 2} & \widetilde{\mathbf{C}}_{2}(\widetilde{\mathbf{t}}, \widetilde{\mathbf{z}}, \mathbf{v}) \mathbf{M}^{1 / 2} \\
\mathbf{M}^{1 / 2}
\end{array}\right] \\
& \cdot\left(\left[\begin{array}{cc}
\widetilde{\mathbf{a}}(\widetilde{\mathbf{z}}, \mathbf{v}) \\
\mathbf{v}
\end{array}\right]-\left[\begin{array}{cc}
\widetilde{\mathbf{B}}(\widetilde{\mathbf{z}}, \mathbf{v}) & \mathbf{O}_{p_{1} D \times p_{3}} \\
\mathbf{O}_{p_{3} \times\left(p_{2}+p_{4}\right) D} & \mathbf{I}_{p_{3}}
\end{array}\right] \cdot \overline{\tilde{\mathbf{t}}}\right) \\
&= {\left[\begin{array}{cc}
\widetilde{\mathbf{C}}_{1}(\widetilde{\mathbf{t}}, \widetilde{\mathbf{z}}, \mathbf{v}) \widetilde{\mathbf{N}}^{1 / 2} & \widetilde{\mathbf{C}}_{2}(\widetilde{\mathbf{t}}, \widetilde{\mathbf{z}}, \mathbf{v}) \mathbf{M}^{1 / 2} \\
\mathbf{O}_{p_{3} \times p_{1} D} & \mathbf{M}^{1 / 2}
\end{array}\right]^{\dagger} } \\
& \cdot(\overline{\widetilde{\mathbf{a}}}(\widetilde{\mathbf{z}}, \mathbf{v})-\overline{\widetilde{\mathbf{B}}}(\widetilde{\mathbf{z}}, \mathbf{v}) \overline{\tilde{\mathbf{t}}}),
\end{aligned}
$$

where $(\cdot)^{\dagger}$ represents the Moore-Penrose inverse. Since $\widetilde{\mathbf{C}}_{1}(\mathfrak{t}, \widetilde{\mathbf{z}}, \mathbf{v})$ is invertible, it can be checked that $\left[\begin{array}{cc}\widetilde{\mathbf{C}}_{1}(\tilde{t}, \tilde{z}, \mathbf{v}) \widetilde{\mathbf{N}}^{1 / 2} & \widetilde{\mathbf{C}}_{2}(\mathfrak{t}, \widetilde{z}, \mathbf{v}) \mathbf{M}^{1 / 2} \\ \mathbf{O}_{p_{3} \times p_{1} D} & \mathbf{M}^{1 / 2}\end{array}\right]$ has full row rank, which leads to

$$
\begin{aligned}
& \left(\left[\begin{array}{cc}
\widetilde{\mathbf{C}}_{1}(\widetilde{\mathbf{t}}, \widetilde{\mathbf{z}}, \mathbf{v}) \widetilde{\mathbf{N}}^{1 / 2} & \widetilde{\mathbf{C}}_{2}(\widetilde{\mathbf{t}}, \widetilde{\mathbf{z}}, \mathbf{v}) \mathbf{M}^{1 / 2} \\
\mathbf{O}_{p_{3} \times p_{1} D} & \mathbf{M}^{1 / 2}
\end{array}\right]^{\dagger}\right)^{T} \\
& \cdot\left[\begin{array}{cc}
\widetilde{\mathbf{C}}_{1}(\widetilde{\mathbf{t}}, \widetilde{\mathbf{z}}, \mathbf{v}) \widetilde{\mathbf{N}}^{1 / 2} & \widetilde{\mathbf{C}}_{2}(\widetilde{\mathbf{t}}, \widetilde{\mathbf{z}}, \mathbf{v}) \mathbf{M}^{1 / 2} \\
\mathbf{O}_{p_{3} \times p_{1} D} & \mathbf{M}^{1 / 2}
\end{array}\right]^{\dagger} \\
& =\left(\left[\begin{array}{cc}
\widetilde{\mathbf{C}}_{1}(\widetilde{\mathbf{t}}, \widetilde{\mathbf{z}}, \mathbf{v}) \widetilde{\mathbf{N}}^{1 / 2} & \widetilde{\mathbf{C}}_{2}(\widetilde{\mathbf{t}}, \widetilde{\mathbf{z}}, \mathbf{v}) \mathbf{M}^{1 / 2} \\
\mathbf{O}_{p_{3} \times p_{1} D} & \mathbf{M}^{1 / 2}
\end{array}\right]\right. \\
& \left.\cdot\left[\begin{array}{cc}
\widetilde{\mathbf{C}}_{1}(\widetilde{\mathbf{t}}, \widetilde{\mathbf{z}}, \mathbf{v}) \widetilde{\mathbf{N}}^{1 / 2} & \widetilde{\mathbf{C}}_{2}(\widetilde{\mathbf{t}}, \widetilde{\mathbf{z}}, \mathbf{v}) \mathbf{M}^{1 / 2} \\
\mathbf{O}_{p_{3} \times p_{1} D} & \mathbf{M}^{1 / 2}
\end{array}\right]^{T}\right)^{-1} \\
& =(\overline{\widetilde{\mathbf{Q}}}(\widetilde{\mathbf{t}}, \widetilde{\mathbf{z}}, \mathbf{v}))^{-1} \text {. }
\end{aligned}
$$

Combining (20) and (21) yields

$$
\begin{aligned}
\left\|\left[\begin{array}{c}
\widetilde{\mathbf{n}}^{\prime} \\
\mathbf{m}^{\prime}
\end{array}\right]_{\mathrm{opt}}\right\|_{2}^{2}=(\overline{\widetilde{\mathbf{a}}}(\widetilde{\mathbf{z}}, \mathbf{v})-\overline{\widetilde{\mathbf{B}}}(\widetilde{\mathbf{z}}, \mathbf{v}) \overline{\widetilde{\mathbf{t}}})^{T}(\overline{\widetilde{\mathbf{Q}}}(\widetilde{\mathbf{t}}, \widetilde{\mathbf{z}}, \mathbf{v}))^{-1} \\
\cdot(\overline{\overline{\mathbf{a}}}(\widetilde{\mathbf{z}}, \mathbf{v})-\overline{\widetilde{\mathbf{B}}}(\widetilde{\mathbf{z}}, \mathbf{v}) \overline{\widetilde{\mathbf{t}}})
\end{aligned}
$$

which, combined with (19), proves the proposition.

We would like to emphasize that (16) is the CTLS optimization model to jointly estimate source position $\widetilde{\mathbf{u}}$ and system parameter $\mathbf{w}$ simultaneously. Moreover, it is a generic model that can be applied to many different location measurements. In the next section, the numerical algorithm to solve (16) is derived.

\section{Numerical Algorithm}

It is obvious that (16) is a nonlinear minimization problem. Therefore, the analytical solution is in general not available, and a numerical technique is required. One widely applied numerical method is Newton iteration, which has two-order convergence rate if the function to be minimized is twice differentiable. Note that, in each iteration step, the gradient and Hessian matrix of the object function must be computed. Hence, we need to derive the explicit expressions for the gradient and Hessian matrix.

For notational convenience, the cost function $J_{\text {ctls }}(\widetilde{\mathbf{u}}, \mathbf{w})$ in (16) is rewritten as

$$
J_{\mathrm{ctls}}(\widetilde{\mathbf{u}}, \mathbf{w})=(\mathbf{g}(\widetilde{\mathbf{u}}, \mathbf{w}))^{T} \mathbf{G}(\widetilde{\mathbf{u}}, \mathbf{w}) \mathbf{g}(\widetilde{\mathbf{u}}, \mathbf{w}),
$$

where

$$
\begin{aligned}
\mathbf{g}(\widetilde{\mathbf{u}}, \mathbf{w}) & =\overline{\widetilde{\mathbf{B}}}(\widetilde{\mathbf{z}}, \mathbf{v}) \overline{\widetilde{\mathbf{t}}}-\overline{\widetilde{\mathbf{a}}}(\widetilde{\mathbf{z}}, \mathbf{v}), \\
\mathbf{G}(\widetilde{\mathbf{u}}, \mathbf{w}) & =(\overline{\widetilde{\mathbf{Q}}}(\widetilde{\mathbf{t}}, \widetilde{\mathbf{z}}, \mathbf{v}))^{-1} .
\end{aligned}
$$

From (23), the gradient of $J_{\text {ctls }}(\widetilde{\mathbf{u}}, \mathbf{w})$ can be expressed as

$$
\boldsymbol{\varphi}(\widetilde{\mathbf{u}}, \mathbf{w})=\left[\frac{\frac{\partial J_{\mathrm{ctls}}(\widetilde{\mathbf{u}}, \mathbf{w})}{\partial \widetilde{\mathbf{u}}}}{\frac{\partial J_{\mathrm{ctls}}(\widetilde{\mathbf{u}}, \mathbf{w})}{\partial \mathbf{w}}}\right]=\varphi_{1}(\widetilde{\mathbf{u}}, \mathbf{w})+\varphi_{2}(\widetilde{\mathbf{u}}, \mathbf{w}),
$$

where

$$
\begin{aligned}
& \boldsymbol{\varphi}_{1}(\widetilde{\mathbf{u}}, \mathbf{w})=\left[\frac{2\left(\frac{\partial \mathbf{g}(\widetilde{\mathbf{u}}, \mathbf{w})}{\partial \widetilde{\mathbf{u}}^{T}}\right)^{T} \mathbf{G}(\widetilde{\mathbf{u}}, \mathbf{w}) \mathbf{g}(\widetilde{\mathbf{u}}, \mathbf{w})}{2\left(\frac{\partial \mathbf{g}(\widetilde{\mathbf{u}}, \mathbf{w})}{\partial \mathbf{w}^{T}}\right)^{T} \mathbf{G}(\widetilde{\mathbf{u}}, \mathbf{w}) \mathbf{g}(\widetilde{\mathbf{u}}, \mathbf{w})}\right] \\
& \boldsymbol{\varphi}_{2}(\widetilde{\mathbf{u}}, \mathbf{w}) \\
& =\left[\frac{\left(\frac{\partial \operatorname{vec}(\mathbf{G}(\widetilde{\mathbf{u}}, \mathbf{w}))}{\partial \widetilde{\mathbf{u}}^{T}}\right)^{T}(\mathbf{g}(\widetilde{\mathbf{u}}, \mathbf{w}) \otimes \mathbf{g}(\widetilde{\mathbf{u}}, \mathbf{w}))}{\left(\frac{\partial \operatorname{vec}(\mathbf{G}(\widetilde{\mathbf{u}}, \mathbf{w}))}{\partial \mathbf{w}^{T}}\right)^{T}(\mathbf{g}(\widetilde{\mathbf{u}}, \mathbf{w}) \otimes \mathbf{g}(\widetilde{\mathbf{u}}, \mathbf{w}))}\right] .
\end{aligned}
$$

Applying (25), the Hessian matrix of $J_{\text {ctls }}(\widetilde{\mathbf{u}}, \mathbf{w})$ is given by

$$
\begin{aligned}
\Psi(\widetilde{\mathbf{u}}, \mathbf{w}) & =\left[\frac{\partial \varphi(\widetilde{\mathbf{u}}, \mathbf{w})}{\partial \widetilde{\mathbf{u}}^{T}} \mid \frac{\partial \varphi(\widetilde{\mathbf{u}}, \mathbf{w})}{\partial \mathbf{w}^{T}}\right] \\
& =\left[\begin{array}{l|l}
\frac{\partial^{2} J_{\mathrm{ctls}}(\widetilde{\mathbf{u}}, \mathbf{w})}{\partial \widetilde{\mathbf{u}} \partial \widetilde{\widetilde{u}}^{T}} & \frac{\partial^{2} J_{\mathrm{ctls}}(\widetilde{\mathbf{u}}, \mathbf{w})}{\partial \widetilde{\mathbf{u}} \partial \mathbf{w}^{T}} \\
\hline \frac{\partial^{2} J_{\mathrm{ctls}}(\widetilde{\mathbf{u}}, \mathbf{w})}{\partial \mathbf{w} \partial \widetilde{\mathbf{u}}^{T}} & \frac{\partial^{2} J_{\mathrm{ctls}}(\widetilde{\mathbf{u}}, \mathbf{w})}{\partial \mathbf{w} \partial \mathbf{w}^{T}}
\end{array}\right]
\end{aligned}
$$




$$
=\Psi_{1}(\widetilde{\mathbf{u}}, \mathbf{w})+\Psi_{2}(\widetilde{\mathbf{u}}, \mathbf{w}),
$$

where

$$
\begin{aligned}
\Psi_{1}(\widetilde{\mathbf{u}}, \mathbf{w}) & =\left[\frac{\partial \varphi_{1}(\widetilde{\mathbf{u}}, \mathbf{w})}{\partial \widetilde{\mathbf{u}}^{T}} \mid \frac{\partial \varphi_{1}(\widetilde{\mathbf{u}}, \mathbf{w})}{\partial \mathbf{w}^{T}}\right] \\
& =\left[\boldsymbol{\Psi}_{1,1}(\widetilde{\mathbf{u}}, \mathbf{w}) \mid \Psi_{1,2}(\widetilde{\mathbf{u}}, \mathbf{w})\right],
\end{aligned}
$$

$$
\begin{aligned}
\boldsymbol{\Psi}_{2}(\widetilde{\mathbf{u}}, \mathbf{w}) & =\left[\frac{\partial \boldsymbol{\varphi}_{2}(\widetilde{\mathbf{u}}, \mathbf{w})}{\partial \widetilde{\mathbf{u}}^{T}} \mid \frac{\partial \boldsymbol{\varphi}_{2}(\widetilde{\mathbf{u}}, \mathbf{w})}{\partial \mathbf{w}^{T}}\right] \\
& =\left[\boldsymbol{\Psi}_{2,1}(\widetilde{\mathbf{u}}, \mathbf{w}) \mid \Psi_{2,2}(\widetilde{\mathbf{u}}, \mathbf{w})\right]
\end{aligned}
$$

in which $\Psi_{1,1}(\widetilde{\mathbf{u}}, \mathbf{w})=\partial \boldsymbol{\varphi}_{1}(\widetilde{\mathbf{u}}, \mathbf{w}) / \partial \widetilde{\mathbf{u}}^{T}, \Psi_{1,2}(\widetilde{\mathbf{u}}, \mathbf{w})=\partial \boldsymbol{\varphi}_{1}(\widetilde{\mathbf{u}}$, $\mathbf{w}) / \partial \mathbf{w}^{T}, \Psi_{2,1}(\widetilde{\mathbf{u}}, \mathbf{w})=\partial \boldsymbol{\varphi}_{2}(\widetilde{\mathbf{u}}, \mathbf{w}) / \partial \widetilde{\mathbf{u}}^{T}$, and $\Psi_{2,2}(\widetilde{\mathbf{u}}, \mathbf{w})=$ $\partial \boldsymbol{\varphi}_{2}(\widetilde{\mathbf{u}}, \mathbf{w}) / \partial \mathbf{w}^{T}$. It follows from (26) that

$$
\begin{aligned}
& \Psi_{1,1}(\widetilde{\mathbf{u}}, \mathbf{w}) \\
& =\left[\frac{2\left(\mathbf{g}(\widetilde{\mathbf{u}}, \mathbf{w}) \otimes \frac{\partial \mathbf{g}(\widetilde{\mathbf{u}}, \mathbf{w})}{\partial \widetilde{\mathbf{u}}^{T}}\right)^{T} \cdot \frac{\partial \operatorname{vec}(\mathbf{G}(\widetilde{\mathbf{u}}, \mathbf{w}))}{\partial \widetilde{\mathbf{u}}^{T}}+2\left(\frac{\partial \mathbf{g}(\widetilde{\mathbf{u}}, \mathbf{w})}{\partial \widetilde{\mathbf{u}}^{T}}\right)^{T} \mathbf{G}(\widetilde{\mathbf{u}}, \mathbf{w}) \cdot \frac{\partial \mathbf{g}(\widetilde{\mathbf{u}}, \mathbf{w})}{\partial \widetilde{\mathbf{u}}^{T}}+2\left(\left((\mathbf{g}(\widetilde{\mathbf{u}}, \mathbf{w}))^{T} \mathbf{G}(\widetilde{\mathbf{u}}, \mathbf{w})\right) \otimes \mathbf{I}_{p_{2} D}\right)\left(\frac{\partial}{\partial \widetilde{\mathbf{u}}^{T}} \operatorname{vec}\left(\left(\frac{\partial \mathbf{g}(\widetilde{\mathbf{u}}, \mathbf{w})}{\partial \widetilde{\mathbf{u}}^{T}}\right)^{T}\right)\right)}{2\left(\mathbf{g}(\widetilde{\mathbf{u}}, \mathbf{w}) \otimes \frac{\partial \mathbf{g}(\widetilde{\mathbf{u}}, \mathbf{w})}{\partial \mathbf{w}^{T}}\right)^{T} \cdot \frac{\partial \operatorname{vec}(\mathbf{G}(\widetilde{\mathbf{u}}, \mathbf{w}))}{\partial \widetilde{\mathbf{u}}^{T}}+2\left(\frac{\partial \mathbf{g}(\widetilde{\mathbf{u}}, \mathbf{w})}{\partial \mathbf{w}^{T}}\right)^{T} \mathbf{G}(\widetilde{\mathbf{u}}, \mathbf{w}) \cdot \frac{\partial \mathbf{g}(\widetilde{\mathbf{u}}, \mathbf{w})}{\partial \widetilde{\mathbf{u}}^{T}}+2\left(\left((\mathbf{g}(\widetilde{\mathbf{u}}, \mathbf{w}))^{T} \mathbf{G}(\widetilde{\mathbf{u}}, \mathbf{w})\right) \otimes \mathbf{I}_{p_{3}}\right)\left(\frac{\partial}{\partial \widetilde{\mathbf{u}}^{T}} \operatorname{vec}\left(\left(\frac{\partial \mathbf{g}(\widetilde{\mathbf{u}}, \mathbf{w})}{\partial \mathbf{w}^{T}}\right)^{T}\right)\right)}\right] \\
& \Psi_{1,2}(\widetilde{\mathbf{u}}, \mathbf{w}) \\
& =\left[\frac{2\left(\mathbf{g}(\widetilde{\mathbf{u}}, \mathbf{w}) \otimes \frac{\partial \mathbf{g}(\widetilde{\mathbf{u}}, \mathbf{w})}{\partial \widetilde{\mathbf{u}}^{T}}\right)^{T} \cdot \frac{\partial \operatorname{vec}(\mathbf{G}(\widetilde{\mathbf{u}}, \mathbf{w}))}{\partial \mathbf{w}^{T}}+2\left(\frac{\partial \mathbf{g}(\widetilde{\mathbf{u}}, \mathbf{w})}{\partial \widetilde{\mathbf{u}}^{T}}\right)^{T} \mathbf{G}(\widetilde{\mathbf{u}}, \mathbf{w}) \cdot \frac{\partial \mathbf{g}(\widetilde{\mathbf{u}}, \mathbf{w})}{\partial \mathbf{w}^{T}}+2\left(\left((\mathbf{g}(\widetilde{\mathbf{u}}, \mathbf{w}))^{T} \mathbf{G}(\widetilde{\mathbf{u}}, \mathbf{w})\right) \otimes \mathbf{I}_{p_{2} D}\right)\left(\frac{\partial}{\partial \mathbf{w}^{T}} \operatorname{vec}\left(\left(\frac{\partial \mathbf{g}(\widetilde{\mathbf{u}}, \mathbf{w})}{\partial \widetilde{\mathbf{u}}^{T}}\right)^{T}\right)\right)}{2\left(\mathbf{g}(\widetilde{\mathbf{u}}, \mathbf{w}) \otimes \frac{\partial \mathbf{g}(\widetilde{\mathbf{u}}, \mathbf{w})}{\partial \mathbf{w}^{T}}\right)^{T} \cdot \frac{\partial \operatorname{vec}(\mathbf{G}(\widetilde{\mathbf{u}}, \mathbf{w}))}{\partial \mathbf{w}^{T}}+2\left(\frac{\partial \mathbf{g}(\widetilde{\mathbf{u}}, \mathbf{w})}{\partial \mathbf{w}^{T}}\right)^{T} \mathbf{G}(\widetilde{\mathbf{u}}, \mathbf{w}) \cdot \frac{\partial \mathbf{g}(\widetilde{\mathbf{u}}, \mathbf{w})}{\partial \mathbf{w}^{T}}+2\left(\left((\mathbf{g}(\widetilde{\mathbf{u}}, \mathbf{w}))^{T} \mathbf{G}(\widetilde{\mathbf{u}}, \mathbf{w})\right) \otimes \mathbf{I}_{p_{3}}\right)\left(\frac{\partial}{\partial \mathbf{w}^{T}} \operatorname{vec}\left(\left(\frac{\partial \mathbf{g}(\widetilde{\mathbf{u}}, \mathbf{w})}{\partial \mathbf{w}^{T}}\right)^{T}\right)\right)},\right. \\
& \Psi_{2,1}(\widetilde{\mathbf{u}}, \mathbf{w}) \approx\left[\frac{\left(\frac{\partial \operatorname{vec}(\mathbf{G}(\widetilde{\mathbf{u}}, \mathbf{w}))}{\partial \widetilde{\mathbf{u}}^{T}}\right)^{T}\left(\left(\mathbf{I}_{p_{1} D+p_{3}} \otimes \mathbf{g}(\widetilde{\mathbf{u}}, \mathbf{w})\right) \cdot \frac{\partial \mathbf{g}(\widetilde{\mathbf{u}}, \mathbf{w})}{\partial \widetilde{\mathbf{u}}^{T}}+\mathbf{g}(\widetilde{\mathbf{u}}, \mathbf{w}) \otimes \frac{\partial \mathbf{g}(\widetilde{\mathbf{u}}, \mathbf{w})}{\partial \widetilde{\mathbf{u}}^{T}}\right)}{\left(\frac{\partial \operatorname{vec}(\mathbf{G}(\widetilde{\mathbf{u}}, \mathbf{w}))}{\partial \mathbf{w}^{T}}\right)^{T}\left(\left(\mathbf{I}_{p_{1} D+p_{3}} \otimes \mathbf{g}(\widetilde{\mathbf{u}}, \mathbf{w})\right) \cdot \frac{\partial \mathbf{g}(\widetilde{\mathbf{u}}, \mathbf{w})}{\partial \widetilde{\mathbf{u}}^{T}}+\mathbf{g}(\widetilde{\mathbf{u}}, \mathbf{w}) \otimes \frac{\partial \mathbf{g}(\widetilde{\mathbf{u}}, \mathbf{w})}{\partial \widetilde{\mathbf{u}}^{T}}\right)}\right] \\
& \Psi_{2,2}(\widetilde{\mathbf{u}}, \mathbf{w}) \approx\left[\frac{\left(\frac{\partial \operatorname{vec}(\mathbf{G}(\widetilde{\mathbf{u}}, \mathbf{w}))}{\partial \widetilde{\mathbf{u}}^{T}}\right)^{T}\left(\left(\mathbf{I}_{p_{1} D+p_{3}} \otimes \mathbf{g}(\widetilde{\mathbf{u}}, \mathbf{w})\right) \cdot \frac{\partial \mathbf{g}(\widetilde{\mathbf{u}}, \mathbf{w})}{\partial \mathbf{w}^{T}}+\mathbf{g}(\widetilde{\mathbf{u}}, \mathbf{w}) \otimes \frac{\partial \mathbf{g}(\widetilde{\mathbf{u}}, \mathbf{w})}{\partial \mathbf{w}^{T}}\right)}{\left(\frac{\partial \operatorname{vec}(\mathbf{G}(\widetilde{\mathbf{u}}, \mathbf{w}))}{\partial \mathbf{w}^{T}}\right)^{T}\left(\left(\mathbf{I}_{p_{1} D+p_{3}} \otimes \mathbf{g}(\widetilde{\mathbf{u}}, \mathbf{w})\right) \cdot \frac{\partial \mathbf{g}(\widetilde{\mathbf{u}}, \mathbf{w})}{\partial \mathbf{w}^{T}}+\mathbf{g}(\widetilde{\mathbf{u}}, \mathbf{w}) \otimes \frac{\partial \mathbf{g}(\widetilde{\mathbf{u}}, \mathbf{w})}{\partial \mathbf{w}^{T}}\right)}\right] .
\end{aligned}
$$

It is worth pointing out that all the quadratic terms of $\mathbf{g}(\widetilde{\mathbf{u}}, \mathbf{w})$ are ignored in (31) and (32). The reason is that these terms hardly affect the convergence rate and asymptotic performance of the CTLS method.

Based on the above discussion, the Newton iteration is given by

$$
\begin{aligned}
{\left[\begin{array}{c}
\widehat{\widetilde{\mathbf{u}}}^{(k+1)} \\
\widehat{\mathbf{w}}^{(k+1)}
\end{array}\right]=} & {\left[\begin{array}{l}
\widehat{\widetilde{\mathbf{u}}}^{(k)} \\
\widehat{\mathbf{w}}^{(k)}
\end{array}\right] } \\
& -\mu^{k}\left(\Psi\left(\widehat{\widetilde{\mathbf{u}}}^{(k)}, \widehat{\mathbf{w}}^{(k)}\right)\right)^{-1} \boldsymbol{\varphi}\left(\widehat{\widehat{\mathbf{u}}}^{(k)}, \widehat{\mathbf{w}}^{(k)}\right),
\end{aligned}
$$

where the subscript $(k)$ denotes the $k$ th iteration and $\mu(0<$ $\mu<1)$ is a suitable step size. Some remarks on the Newton iteration follow.

Remark 2. The initial value for the iteration can be obtained by the WLS or TLS methods, both of which can provide an approximate closed-form solution.

Remark 3. $\left\|\varphi\left(\widehat{\widetilde{\mathbf{u}}}^{(k)}, \widehat{\mathbf{w}}^{(k)}\right)\right\|_{2} \leq \xi$ can be used as the prescribed convergence criterion.
Remark 4. Note that, in (29)-(32), there exist some matrices whose expressions are not yet specified. They include

$$
\begin{aligned}
& \mathbf{Z}_{1}=\frac{\partial \mathbf{g}(\widetilde{\mathbf{u}}, \mathbf{w})}{\partial \widetilde{\mathbf{u}}^{T}}, \\
& \mathbf{Z}_{2}=\frac{\partial \mathbf{g}(\widetilde{\mathbf{u}}, \mathbf{w})}{\partial \mathbf{w}^{T}}, \\
& \mathbf{Z}_{3}=\frac{\partial}{\partial \widetilde{\mathbf{u}}^{T}} \operatorname{vec}\left(\left(\frac{\partial \mathbf{g}(\widetilde{\mathbf{u}}, \mathbf{w})}{\partial \widetilde{\mathbf{u}}^{T}}\right)^{T}\right) \\
& \mathbf{Z}_{4}=\frac{\partial}{\partial \mathbf{w}^{T}} \operatorname{vec}\left(\left(\frac{\partial \mathbf{g}(\widetilde{\mathbf{u}}, \mathbf{w})}{\partial \widetilde{\mathbf{u}}^{T}}\right)^{T}\right), \\
& \mathbf{Z}_{5}=\frac{\partial}{\partial \widetilde{\mathbf{u}}^{T}} \operatorname{vec}\left(\left(\frac{\partial \mathbf{g}(\widetilde{\mathbf{u}}, \mathbf{w})}{\partial \mathbf{w}^{T}}\right)^{T}\right) \\
& \mathbf{Z}_{6}=\frac{\partial}{\partial \mathbf{w}^{T}} \operatorname{vec}\left(\left(\frac{\partial \mathbf{g}(\widetilde{\mathbf{u}}, \mathbf{w})}{\partial \mathbf{w}^{T}}\right)^{T}\right), \\
& \mathbf{Z}_{7}=\frac{\partial \mathrm{vec}(\mathbf{G}(\widetilde{\mathbf{u}}, \mathbf{w}))}{\partial \widetilde{\mathbf{u}} T}, \\
& \mathbf{Z}_{8}=\frac{\partial \operatorname{vec}(\mathbf{G}(\widetilde{\mathbf{u}}, \mathbf{w}))}{\partial \mathbf{w}^{T}} .
\end{aligned}
$$


The exact expressions for the eight matrices in (34) are provided in Appendix A.

Remark 5. Since the weighting matrix $\mathbf{G}(\widetilde{\mathbf{u}}, \mathbf{w})=(\overline{\widetilde{\mathbf{Q}}} \tilde{\mathbf{t}}, \widetilde{\mathbf{z}}$, $\mathbf{v}))^{-1}$ is updated at each iteration step, the proposed CTLS solution is able to yield much smaller estimation bias compared to the TLS and TWLS solutions, as discussed in Section 7.

\section{Performance Analysis}

In this section, the analytical expression for the covariance matrix of the above CTLS estimator is derived. Furthermore, the CTLS solution is proved theoretically to reach the CRB accuracy before the thresholding effect starts to take place.

5.1. Covariance Matrix of the CTLS Solution. Assuming the convergence results for the Newton iteration are denoted by $\widehat{\widetilde{\mathbf{u}}}_{\mathrm{ctls}}$ and $\widehat{\mathbf{w}}_{\mathrm{ctls}}$, it follows from the iteration termination criteria given in Remark 3 that

$$
\begin{aligned}
& \lim _{k \rightarrow+\infty} \varphi\left(\widehat{\widetilde{\mathbf{u}}}^{(k)}, \widehat{\mathbf{w}}^{(k)}\right)=\boldsymbol{\varphi}\left(\widehat{\widetilde{\mathbf{u}}}_{\mathrm{ctls}}, \widehat{\mathbf{w}}_{\mathrm{ctls}}\right) \\
& =\left[\frac{\left.\frac{\partial J_{\mathrm{ctls}}\left(\widetilde{\mathbf{u}}, \widehat{\mathbf{w}}_{\mathrm{ctls}}\right)}{\partial \widetilde{\mathbf{u}}}\right|_{\widetilde{\mathbf{u}}=\widehat{\widetilde{\mathbf{u}}}_{\mathrm{cts}}}}{\left.\frac{\partial J_{\mathrm{ctls}}\left(\widehat{\widetilde{\mathbf{u}}}_{\mathrm{ctls}}, \mathbf{w}\right)}{\partial \mathbf{w}}\right|_{\mathbf{w}=\widehat{\mathbf{w}}_{\mathrm{ctls}}}}\right] \\
& =\mathbf{O}_{\left(p_{2} D+p_{3}\right) \times 1} .
\end{aligned}
$$

The substitution of (25)-(26) into (35) leads to

$$
\begin{aligned}
& \mathbf{O}_{\left(p_{2} D+p_{3}\right) \times 1}
\end{aligned}
$$

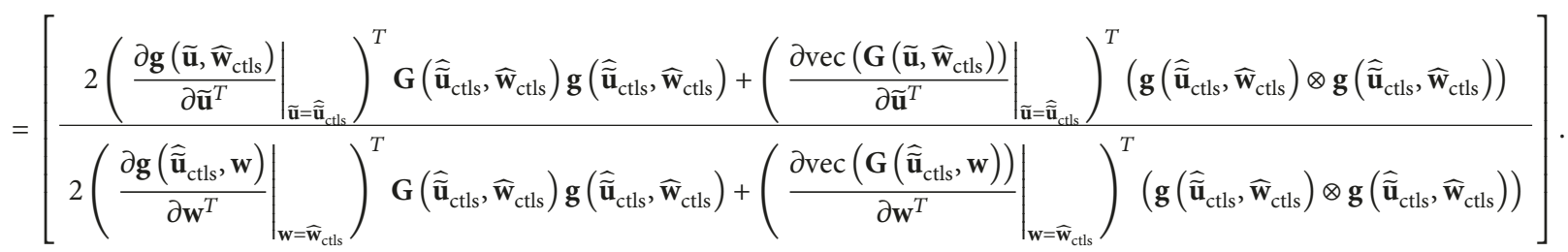

Performing a first-order Taylor series expansion of $\mathbf{g}\left(\widehat{\widetilde{\mathbf{u}}}_{\mathrm{ctls}}, \widehat{\mathbf{w}}_{\mathrm{ctls}}\right)$ around the true values $\widetilde{\mathbf{u}}$ and $\mathbf{w}$ produces

$$
\begin{aligned}
\mathbf{g}\left(\widetilde{\widetilde{\mathbf{u}}}_{\mathrm{ctls}}, \widehat{\mathbf{w}}_{\mathrm{ctls}}\right) & \approx\left[\begin{array}{c}
\widetilde{\mathbf{B}}\left(\widetilde{\mathbf{z}}_{0}, \mathbf{w}\right) \widetilde{\mathbf{H}}_{1}(\widetilde{\mathbf{u}}, \mathbf{w}) \cdot \boldsymbol{\delta} \widetilde{\mathbf{u}}_{\mathrm{ctls}}+\widetilde{\mathbf{B}}\left(\widetilde{\mathbf{z}}_{0}, \mathbf{w}\right) \widetilde{\mathbf{H}}_{2}(\widetilde{\mathbf{u}}, \mathbf{w}) \cdot \boldsymbol{\delta} \mathbf{w}_{\mathrm{ctls}}-\widetilde{\mathbf{C}}_{1}\left(\widetilde{\mathbf{t}}, \widetilde{\mathbf{z}}_{0}, \mathbf{w}\right) \widetilde{\mathbf{n}}-\widetilde{\mathbf{C}}_{2}\left(\widetilde{\mathbf{t}}, \widetilde{\mathbf{z}}_{0}, \mathbf{w}\right) \mathbf{m} \\
\boldsymbol{\delta} \mathbf{w}_{\mathrm{ctls}}-\mathbf{m}
\end{array}\right] \\
& =\left[\begin{array}{cc}
\widetilde{\mathbf{B}}\left(\widetilde{\mathbf{z}}_{0}, \mathbf{w}\right) \widetilde{\mathbf{H}}_{1}(\widetilde{\mathbf{u}}, \mathbf{w}) & \widetilde{\mathbf{B}}\left(\widetilde{\mathbf{z}}_{0}, \mathbf{w}\right) \widetilde{\mathbf{H}}_{2}(\widetilde{\mathbf{u}}, \mathbf{w}) \\
\mathbf{O}_{p_{3} \times p_{2} D} & \mathbf{I}_{p_{3}}
\end{array}\right] \cdot\left[\begin{array}{l}
\boldsymbol{\delta} \widetilde{\mathbf{u}}_{\mathrm{ctls}} \\
\boldsymbol{\delta} \mathbf{w}_{\mathrm{ctls}}
\end{array}\right]-\left[\begin{array}{cc}
\widetilde{\mathbf{C}}_{1}\left(\widetilde{\mathbf{t}}_{,}, \widetilde{\mathbf{z}}_{0}, \mathbf{w}\right) & \widetilde{\mathbf{C}}_{2}\left(\widetilde{\mathbf{t}}, \widetilde{\mathbf{z}}_{0}, \mathbf{w}\right) \\
\mathbf{O}_{p_{3} \times p_{1} D} & \mathbf{I}_{p_{3}}
\end{array}\right] \cdot\left[\begin{array}{l}
\widetilde{\mathbf{n}} \\
\mathbf{m}
\end{array}\right],
\end{aligned}
$$

where $\boldsymbol{\delta} \widetilde{\mathbf{u}}_{\mathrm{ctls}}=\widehat{\widetilde{\mathbf{u}}}_{\mathrm{ctls}}-\widetilde{\mathbf{u}}$ and $\boldsymbol{\delta} \mathbf{w}_{\mathrm{ctls}}=\widehat{\mathbf{w}}_{\mathrm{ctls}}-\mathbf{w}$ are estimation errors. Besides, $\widetilde{\mathbf{H}}_{1}(\widetilde{\mathbf{u}}, \mathbf{w})=\partial \widetilde{\mathbf{h}}(\widetilde{\mathbf{u}}, \mathbf{w}) / \partial \widetilde{\mathbf{u}}^{T}$ and $\widetilde{\mathbf{H}}_{2}(\widetilde{\mathbf{u}}, \mathbf{w})=$ $\partial \widetilde{\mathbf{h}}(\widetilde{\mathbf{u}}, \mathbf{w}) / \partial \mathbf{w}^{T}$, whose expressions are given by

$$
\begin{aligned}
& \widetilde{\mathbf{H}}_{1}(\widetilde{\mathbf{u}}, \mathbf{w}) \\
& =\operatorname{blkdiag}\left[\begin{array}{llll}
\mathbf{H}_{1}\left(\mathbf{u}_{1}, \mathbf{w}\right) & \mathbf{H}_{1}\left(\mathbf{u}_{2}, \mathbf{w}\right) & \cdots & \mathbf{H}_{1}\left(\mathbf{u}_{D}, \mathbf{w}\right)
\end{array}\right] \\
& \widetilde{\mathbf{H}}_{2}(\widetilde{\mathbf{u}}, \mathbf{w}) \\
& =\left[\begin{array}{llll}
\left(\mathbf{H}_{2}\left(\mathbf{u}_{1}, \mathbf{w}\right)\right)^{T} & \left(\mathbf{H}_{2}\left(\mathbf{u}_{2}, \mathbf{w}\right)\right)^{T} & \cdots & \left(\mathbf{H}_{2}\left(\mathbf{u}_{D}, \mathbf{w}\right)\right)^{T}
\end{array}\right]^{T},
\end{aligned}
$$

where

$$
\mathbf{H}_{1}\left(\mathbf{u}_{d}, \mathbf{w}\right)=\frac{\partial \mathbf{h}\left(\mathbf{u}_{d}, \mathbf{w}\right)}{\partial \mathbf{u}_{d}^{T}}=\left[\begin{array}{c}
\mathbf{I}_{p_{2}} \\
\mathbf{S}_{1}\left(\mathbf{u}_{d}, \mathbf{w}\right)
\end{array}\right]
$$

$$
\begin{aligned}
& \in \mathbf{R}^{\left(p_{2}+p_{4}\right) \times p_{2}} \\
& \mathbf{H}_{2}\left(\mathbf{u}_{d}, \mathbf{w}\right)=\frac{\partial \mathbf{h}\left(\mathbf{u}_{d}, \mathbf{w}\right)}{\partial \mathbf{w}^{T}}=\left[\begin{array}{c}
-\mathbf{J} \\
\mathbf{S}_{2}\left(\mathbf{u}_{d}, \mathbf{w}\right)
\end{array}\right] \\
& \in \mathbf{R}^{\left(p_{2}+p_{4}\right) \times p_{3}} \\
& \quad(1 \leq d \leq D)
\end{aligned}
$$

in which $\mathbf{S}_{1}\left(\mathbf{u}_{d}, \mathbf{w}\right)=\partial \mathbf{s}\left(\mathbf{u}_{d}, \mathbf{w}\right) / \partial \mathbf{u}_{d}^{T} \in \mathbf{R}^{p_{4} \times p_{2}}$ and $\mathbf{S}_{2}\left(\mathbf{u}_{d}, \mathbf{w}\right)=$ $\partial \mathbf{s}\left(\mathbf{u}_{d}, \mathbf{w}\right) / \partial \mathbf{w}^{T} \in \mathbf{R}^{p_{4} \times p_{3}}$.

Substituting (37) into (36) and omitting the second- and higher-order error terms yields 


$$
\begin{aligned}
& \mathbf{O}_{\left(p_{2} D+p_{3}\right) \times 1} \\
& \approx\left[\begin{array}{cc}
\widetilde{\mathbf{B}}\left(\widetilde{\mathbf{z}}_{0}, \mathbf{w}\right) \widetilde{\mathbf{H}}_{1}(\widetilde{\mathbf{u}}, \mathbf{w}) & \widetilde{\mathbf{B}}\left(\widetilde{\mathbf{z}}_{0}, \mathbf{w}\right) \widetilde{\mathbf{H}}_{2}(\widetilde{\mathbf{u}}, \mathbf{w}) \\
\mathbf{O}_{p_{3} \times p_{2} D} & \mathbf{I}_{p_{3}}
\end{array}\right]^{T} \\
& \cdot \mathbf{G}_{0}(\widetilde{\mathbf{u}}, \mathbf{w}) \\
& \cdot\left[\begin{array}{cc}
\widetilde{\mathbf{B}}\left(\widetilde{\mathbf{z}}_{0}, \mathbf{w}\right) \widetilde{\mathbf{H}}_{1}(\widetilde{\mathbf{u}}, \mathbf{w}) & \widetilde{\mathbf{B}}\left(\widetilde{\mathbf{z}}_{0}, \mathbf{w}\right) \widetilde{\mathbf{H}}_{2}(\widetilde{\mathbf{u}}, \mathbf{w}) \\
\mathbf{O}_{p_{3} \times p_{2} D} & \mathbf{I}_{p_{3}}
\end{array}\right] \\
& \cdot\left[\begin{array}{l}
\delta \widetilde{\mathbf{u}}_{\mathrm{ctls}} \\
\delta \mathbf{w}_{\mathrm{ctls}}
\end{array}\right]
\end{aligned}
$$

$$
\begin{aligned}
& -\left[\begin{array}{cc}
\widetilde{\mathbf{B}}\left(\widetilde{\mathbf{z}}_{0}, \mathbf{w}\right) \widetilde{\mathbf{H}}_{1}(\widetilde{\mathbf{u}}, \mathbf{w}) & \widetilde{\mathbf{B}}\left(\widetilde{\mathbf{z}}_{0}, \mathbf{w}\right) \widetilde{\mathbf{H}}_{2}(\widetilde{\mathbf{u}}, \mathbf{w}) \\
\mathbf{O}_{p_{3} \times p_{2} D} & \mathbf{I}_{p_{3}}
\end{array}\right]^{T} \\
& \cdot \mathbf{G}_{0}(\widetilde{\mathbf{u}}, \mathbf{w}) \cdot\left[\begin{array}{cc}
\widetilde{\mathbf{C}}_{1}\left(\widetilde{\mathbf{t}}, \widetilde{\mathbf{z}}_{0}, \mathbf{w}\right) & \widetilde{\mathbf{C}}_{2}\left(\widetilde{\mathbf{t}}, \widetilde{\mathbf{z}}_{0}, \mathbf{w}\right) \\
\mathbf{O}_{p_{3} \times p_{1} D} & \mathbf{I}_{p_{3}}
\end{array}\right] \\
& \cdot\left[\begin{array}{c}
\widetilde{\mathbf{n}} \\
\mathbf{m}
\end{array}\right],
\end{aligned}
$$

where

$$
\begin{aligned}
\mathbf{G}_{0}(\widetilde{\mathbf{u}}, \mathbf{w}) & =\left.\mathbf{G}(\widetilde{\mathbf{u}}, \mathbf{w})\right|_{\substack{\mathbf{n}=\mathbf{O}_{p_{1} D \times 1} \\
\mathbf{m}=\mathbf{O}_{p_{3} \times 1}}}=\left(\widetilde{\mathbf{Q}}\left(\widetilde{\mathbf{t}}, \widetilde{\mathbf{z}}_{0}, \mathbf{w}\right)\right)^{-1} \\
& =\left[\begin{array}{c|c}
\widetilde{\mathbf{C}}_{1}\left(\widetilde{\mathbf{t}}, \widetilde{\mathbf{z}}_{0}, \mathbf{w}\right) \widetilde{\mathbf{N}}\left(\widetilde{\mathbf{C}}_{1}\left(\widetilde{\mathbf{t}}, \widetilde{\mathbf{z}}_{0}, \mathbf{w}\right)\right)^{T}+\widetilde{\mathbf{C}}_{2}\left(\widetilde{\mathbf{t}}, \widetilde{\mathbf{z}}_{0}, \mathbf{w}\right) \mathbf{M}\left(\widetilde{\mathbf{C}}_{2}\left(\widetilde{\mathbf{t}}, \widetilde{\mathbf{z}}_{0}, \mathbf{w}\right)\right)^{T} & \widetilde{\mathbf{C}}_{2}\left(\widetilde{\mathbf{t}}, \widetilde{\mathbf{z}}_{0}, \mathbf{w}\right) \mathbf{M} \\
\hline \mathbf{M}\left(\widetilde{\mathbf{C}}_{2}\left(\widetilde{\mathbf{t}}, \widetilde{\mathbf{z}}_{0}, \mathbf{w}\right)\right)^{T} & \mathbf{M}
\end{array}\right]^{-1} .
\end{aligned}
$$

It can be readily deduced from (40) that

$$
\begin{aligned}
& {\left[\begin{array}{l}
\delta \widetilde{\mathbf{u}}_{\mathrm{ctls}} \\
\delta \mathbf{w}_{\mathrm{ctls}}
\end{array}\right]} \\
& \approx\left(\left[\begin{array}{cc}
\widetilde{\mathbf{B}}\left(\widetilde{\mathbf{z}}_{0}, \mathbf{w}\right) \widetilde{\mathbf{H}}_{1}(\widetilde{\mathbf{u}}, \mathbf{w}) & \widetilde{\mathbf{B}}\left(\widetilde{\mathbf{z}}_{0}, \mathbf{w}\right) \widetilde{\mathbf{H}}_{2}(\widetilde{\mathbf{u}}, \mathbf{w}) \\
\mathbf{O}_{p_{3} \times p_{2} D} & \mathbf{I}_{p_{3}}
\end{array}\right]^{T}\right. \\
& \cdot \mathbf{G}_{0}(\widetilde{\mathbf{u}}, \mathbf{w}) \\
& \left.\cdot\left[\begin{array}{cc}
\widetilde{\mathbf{B}}\left(\widetilde{\mathbf{z}}_{0}, \mathbf{w}\right) \widetilde{\mathbf{H}}_{1}(\widetilde{\mathbf{u}}, \mathbf{w}) & \widetilde{\mathbf{B}}\left(\widetilde{\mathbf{z}}_{0}, \mathbf{w}\right) \widetilde{\mathbf{H}}_{2}(\widetilde{\mathbf{u}}, \mathbf{w}) \\
\mathbf{O}_{p_{3} \times p_{2} D} & \mathbf{I}_{p_{3}}
\end{array}\right]\right)^{-1} \\
& \times\left[\begin{array}{cc}
\widetilde{\mathbf{B}}\left(\widetilde{\mathbf{z}}_{0}, \mathbf{w}\right) \widetilde{\mathbf{H}}_{1}(\widetilde{\mathbf{u}}, \mathbf{w}) & \widetilde{\mathbf{B}}\left(\widetilde{\mathbf{z}}_{0}, \mathbf{w}\right) \widetilde{\mathbf{H}}_{2}(\widetilde{\mathbf{u}}, \mathbf{w}) \\
\mathbf{O}_{p_{3} \times p_{2} D} & \mathbf{I}_{p_{3}}
\end{array}\right]^{T} \\
& \cdot \mathbf{G}_{0}(\widetilde{\mathbf{u}}, \mathbf{w}) \cdot\left[\begin{array}{cc}
\widetilde{\mathbf{C}}_{1}\left(\widetilde{\mathbf{t}}, \widetilde{\mathbf{z}}_{0}, \mathbf{w}\right) & \widetilde{\mathbf{C}}_{2}\left(\widetilde{\mathbf{t}}, \widetilde{\mathbf{z}}_{0}, \mathbf{w}\right) \\
\mathbf{O}_{p_{3} \times p_{1} D} & \mathbf{I}_{p_{3}}
\end{array}\right] \cdot\left[\begin{array}{l}
\widetilde{\mathbf{n}} \\
\mathbf{m}
\end{array}\right] .
\end{aligned}
$$

Then, the covariance matrix of estimated vector $\left[\begin{array}{c}\widehat{\widetilde{\mathbf{u}}}_{\text {ctls }} \\ \widehat{\mathbf{w}}_{\text {ctls }}\end{array}\right]$ is given by

$$
\begin{aligned}
& \operatorname{cov}\left(\left[\begin{array}{c}
\widehat{\widetilde{\mathbf{u}}}_{\mathrm{ctls}} \\
\widehat{\mathbf{w}}_{\mathrm{ctls}}
\end{array}\right]\right)=E\left[\left[\begin{array}{l}
\boldsymbol{\delta} \widetilde{\mathbf{u}}_{\mathrm{ctls}} \\
\boldsymbol{\delta} \mathbf{w}_{\mathrm{ctls}}
\end{array}\right] \cdot\left[\begin{array}{l}
\boldsymbol{\delta} \widetilde{\mathbf{u}}_{\mathrm{ctls}} \\
\boldsymbol{\delta} \mathbf{w}_{\mathrm{ctls}}
\end{array}\right]^{T}\right] \\
& =\left(\left[\begin{array}{cc}
\widetilde{\mathbf{B}}\left(\widetilde{\mathbf{z}}_{0}, \mathbf{w}\right) \widetilde{\mathbf{H}}_{1}(\widetilde{\mathbf{u}}, \mathbf{w}) & \widetilde{\mathbf{B}}\left(\widetilde{\mathbf{z}}_{0}, \mathbf{w}\right) \widetilde{\mathbf{H}}_{2}(\widetilde{\mathbf{u}}, \mathbf{w}) \\
\mathbf{O}_{p_{3} \times p_{2} D} & \mathbf{I}_{p_{3}}
\end{array}\right]^{T}\right. \\
& \cdot \mathbf{G}_{0}(\widetilde{\mathbf{u}}, \mathbf{w}) \\
& \left.\cdot\left[\begin{array}{cc}
\widetilde{\mathbf{B}}\left(\widetilde{\mathbf{z}}_{0}, \mathbf{w}\right) \widetilde{\mathbf{H}}_{1}(\widetilde{\mathbf{u}}, \mathbf{w}) & \widetilde{\mathbf{B}}\left(\widetilde{\mathbf{z}}_{0}, \mathbf{w}\right) \widetilde{\mathbf{H}}_{2}(\widetilde{\mathbf{u}}, \mathbf{w}) \\
\mathbf{O}_{p_{3} \times p_{2} D} & \mathbf{I}_{p_{3}}
\end{array}\right]\right)^{-1} .
\end{aligned}
$$

5.2. Asymptotical Efficiency of the CTLS Estimator. In order to prove that the above CTLS solution is asymptotically efficient, it is necessary to obtain the corresponding CRB. According to $[14,16,24,28]$, we have

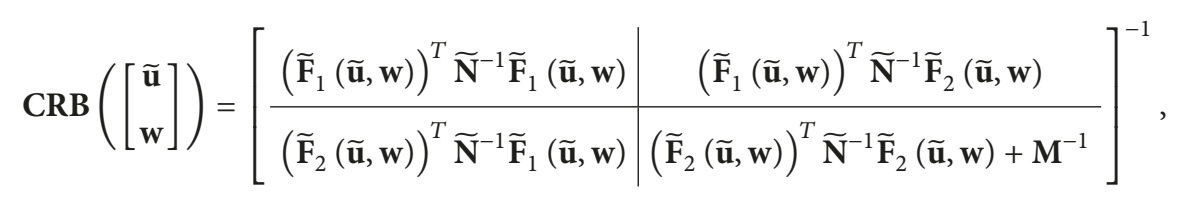

where $\widetilde{\mathbf{F}}_{1}(\widetilde{\mathbf{u}}, \mathbf{w})=\partial \widetilde{\mathbf{f}}(\widetilde{\mathbf{u}}, \mathbf{w}) / \partial \widetilde{\mathbf{u}}^{T}$ and $\widetilde{\mathbf{F}}_{2}(\widetilde{\mathbf{u}}, \mathbf{w})=\partial \widetilde{\mathbf{f}}(\widetilde{\mathbf{u}}, \mathbf{w}) / \partial \mathbf{w}^{T}$. Using the definition of $\widetilde{\mathbf{f}}(\widetilde{\mathbf{u}}, \mathbf{w})$ in (9), it is straightforward to show that

$$
\begin{aligned}
& \widetilde{\mathbf{F}}_{1}(\widetilde{\mathbf{u}}, \mathbf{w}) \\
& =\operatorname{blkdiag}\left[\begin{array}{llll}
\mathbf{F}_{1}\left(\mathbf{u}_{1}, \mathbf{w}\right) & \mathbf{F}_{1}\left(\mathbf{u}_{2}, \mathbf{w}\right) & \cdots & \mathbf{F}_{1}\left(\mathbf{u}_{D}, \mathbf{w}\right)
\end{array}\right]
\end{aligned}
$$




$$
\begin{aligned}
& \in \mathbf{R}^{p_{1} D \times p_{2} D}, \\
& \widetilde{\mathbf{F}}_{2}(\widetilde{\mathbf{u}}, \mathbf{w}) \\
& =\left[\begin{array}{llll}
\left(\mathbf{F}_{2}\left(\mathbf{u}_{1}, \mathbf{w}\right)\right)^{T} & \left(\mathbf{F}_{2}\left(\mathbf{u}_{2}, \mathbf{w}\right)\right)^{T} \cdots\left(\mathbf{F}_{2}\left(\mathbf{u}_{D}, \mathbf{w}\right)\right)^{T}
\end{array}\right]^{T} \\
& \in \mathbf{R}^{p_{1} D \times p_{3}},
\end{aligned}
$$

where $\mathbf{F}_{1}\left(\mathbf{u}_{d}, \mathbf{w}\right)=\partial \mathbf{f}\left(\mathbf{u}_{d}, \mathbf{w}\right) / \partial \mathbf{u}_{d}^{T}$ and $\mathbf{F}_{2}\left(\mathbf{u}_{d}, \mathbf{w}\right)=\partial \mathbf{f}\left(\mathbf{u}_{d}\right.$, $\mathbf{w}) / \partial \mathbf{w}^{T}$.
By comparing (43) and (44), we get the following proposition.

Proposition 6. One has

$$
\operatorname{cov}\left(\left[\begin{array}{c}
\widehat{\tilde{\mathbf{u}}}_{\mathrm{ctls}} \\
\widehat{\mathbf{w}}_{\mathrm{ctls}}
\end{array}\right]\right)=\operatorname{CRB}\left(\left[\begin{array}{c}
\widetilde{\mathbf{u}} \\
\mathbf{w}
\end{array}\right]\right) .
$$

Proof. First, combining (41) and the matrix inversion formulas leads to

$$
\begin{aligned}
& \mathbf{G}_{0}(\widetilde{\mathbf{u}}, \mathbf{w}) \\
& =\left[\begin{array}{c|c}
\left(\widetilde{\mathbf{C}}_{1}\left(\widetilde{\mathbf{t}}, \widetilde{\mathbf{z}}_{0}, \mathbf{w}\right)\right)^{-T} \widetilde{\mathbf{N}}^{-1}\left(\widetilde{\mathbf{C}}_{1}\left(\widetilde{\mathbf{t}}, \widetilde{\mathbf{z}}_{0}, \mathbf{w}\right)\right)^{-1} & -\left(\widetilde{\mathbf{C}}\left(\widetilde{\mathbf{t}}, \widetilde{\mathbf{z}}_{0}, \mathbf{w}\right)\right)^{-T} \widetilde{\mathbf{N}}^{-1}\left(\widetilde{\mathbf{C}}_{1}\left(\widetilde{\mathbf{t}}, \widetilde{\mathbf{z}}_{0}, \mathbf{w}\right)\right)^{-1} \widetilde{\mathbf{C}}_{2}\left(\widetilde{\mathbf{t}}, \widetilde{\mathbf{z}}_{0}, \mathbf{w}\right) \\
\hline-\left(\widetilde{\mathbf{C}}_{2}\left(\widetilde{\mathbf{t}}, \widetilde{\mathbf{z}}_{0}, \mathbf{w}\right)\right)^{T}\left(\widetilde{\mathbf{C}}_{1}\left(\widetilde{\mathbf{t}}, \widetilde{\mathbf{z}}_{0}, \mathbf{w}\right)\right)^{-T} \widetilde{\mathbf{N}}^{-1}\left(\widetilde{\mathbf{C}}_{1}\left(\widetilde{\mathfrak{t}}, \widetilde{\mathbf{z}}_{0}, \mathbf{w}\right)\right)^{-1} & \mathbf{M}^{-1}+\left(\widetilde{\mathbf{C}}_{2}\left(\widetilde{\mathbf{t}}, \widetilde{\mathbf{z}}_{0}, \mathbf{w}\right)\right)^{T}\left(\widetilde{\mathbf{C}}_{1}\left(\widetilde{\mathbf{t}}, \widetilde{\mathbf{z}}_{0}, \mathbf{w}\right)\right)^{-T} \widetilde{\mathbf{N}}^{-1}\left(\widetilde{\mathbf{C}}_{1}\left(\widetilde{\mathbf{t}}, \widetilde{\mathbf{z}}_{0}, \mathbf{w}\right)\right)^{-1} \widetilde{\mathbf{C}}_{2}\left(\widetilde{\mathbf{t}}, \widetilde{\mathbf{z}}_{0}, \mathbf{w}\right)
\end{array}\right] .
\end{aligned}
$$

The proof of (47) is provided in Appendix B. The substitution of (47) into (43) leads to

$$
\operatorname{cov}\left(\left[\begin{array}{c}
\widehat{\widetilde{\mathbf{u}}}_{\mathrm{ctls}} \\
\widehat{\mathbf{w}}_{\mathrm{ctls}}
\end{array}\right]\right)=\left[\begin{array}{ll}
\mathbf{P}_{1} & \mathbf{P}_{2} \\
\mathbf{P}_{2}^{T} & \mathbf{P}_{3}
\end{array}\right]^{-1},
$$

where

$$
\begin{aligned}
\mathbf{P}_{1} & =\left(\widetilde{\mathbf{H}}_{1}(\widetilde{\mathbf{u}}, \mathbf{w})\right)^{T}\left(\widetilde{\mathbf{B}}\left(\widetilde{\mathbf{z}}_{0}, \mathbf{w}\right)\right)^{T}\left(\widetilde{\mathbf{C}}_{1}\left(\widetilde{\mathbf{t}}, \widetilde{\mathbf{z}}_{0}, \mathbf{w}\right)\right)^{-T} \\
& \cdot \widetilde{\mathbf{N}}^{-1}\left(\widetilde{\mathbf{C}}_{1}\left(\widetilde{\mathbf{t}}, \widetilde{\mathbf{z}}_{0}, \mathbf{w}\right)\right)^{-1} \widetilde{\mathbf{B}}\left(\widetilde{\mathbf{z}}_{0}, \mathbf{w}\right) \widetilde{\mathbf{H}}_{1}(\widetilde{\mathbf{u}}, \mathbf{w}), \\
\mathbf{P}_{2} & =\left(\widetilde{\mathbf{H}}_{1}(\widetilde{\mathbf{u}}, \mathbf{w})\right)^{T}\left(\widetilde{\mathbf{B}}\left(\widetilde{\mathbf{z}}_{0}, \mathbf{w}\right)\right)^{T}\left(\widetilde{\mathbf{C}}_{1}\left(\widetilde{\mathbf{t}}, \widetilde{\mathbf{z}}_{0}, \mathbf{w}\right)\right)^{-T} \\
& \cdot \widetilde{\mathbf{N}}^{-1}\left(\widetilde{\mathbf{C}}_{1}\left(\widetilde{\mathbf{t}}, \widetilde{\mathbf{z}}_{0}, \mathbf{w}\right)\right)^{-1} \\
& \cdot\left(\widetilde{\mathbf{B}}\left(\widetilde{\mathbf{z}}_{0}, \mathbf{w}\right) \widetilde{\mathbf{H}}_{2}(\widetilde{\mathbf{u}}, \mathbf{w})-\widetilde{\mathbf{C}}_{2}\left(\widetilde{\mathbf{t}}, \widetilde{\mathbf{z}}_{0}, \mathbf{w}\right)\right) \\
\mathbf{P}_{3} & =\left(\widetilde{\mathbf{B}}\left(\widetilde{\mathbf{z}}_{0}, \mathbf{w}\right) \widetilde{\mathbf{H}}_{2}(\widetilde{\mathbf{u}}, \mathbf{w})-\widetilde{\mathbf{C}}_{2}\left(\widetilde{\mathbf{t}}, \widetilde{\mathbf{z}}_{0}, \mathbf{w}\right)\right)^{T} \\
& \cdot\left(\widetilde{\mathbf{C}}_{1}\left(\widetilde{\mathbf{t}}, \widetilde{\mathbf{z}}_{0}, \mathbf{w}\right)\right)^{-T} \widetilde{\mathbf{N}}^{-1}\left(\widetilde{\mathbf{C}}_{1}\left(\widetilde{\mathbf{t}}, \widetilde{\mathbf{z}}_{0}, \mathbf{w}\right)\right)^{-1} \\
& \cdot\left(\widetilde{\mathbf{B}}\left(\widetilde{\mathbf{z}}_{0}, \mathbf{w}\right) \widetilde{\mathbf{H}}_{2}(\widetilde{\mathbf{u}}, \mathbf{w})-\widetilde{\mathbf{C}}_{2}\left(\widetilde{\mathbf{t}}, \widetilde{\mathbf{z}}_{0}, \mathbf{w}\right)\right)+\mathbf{M}^{-1} .
\end{aligned}
$$

Next, putting $\mathbf{z}_{d 0}=\mathbf{f}\left(\mathbf{u}_{d}, \mathbf{w}\right)$ into (4) produces

$$
\begin{aligned}
\mathbf{a}\left(\mathbf{f}\left(\mathbf{u}_{d}, \mathbf{w}\right), \mathbf{w}\right) & =\mathbf{B}\left(\mathbf{f}\left(\mathbf{u}_{d}, \mathbf{w}\right), \mathbf{w}\right) \mathbf{t}_{d} \\
& =\mathbf{B}\left(\mathbf{f}\left(\mathbf{u}_{d}, \mathbf{w}\right), \mathbf{w}\right) \mathbf{h}\left(\mathbf{u}_{d}, \mathbf{w}\right)
\end{aligned}
$$

Differentiating both sides of (50) with respect to $\mathbf{u}_{d}$ and $\mathbf{w}$, we have

$$
\begin{aligned}
& \mathbf{A}_{1}\left(\mathbf{z}_{d 0}, \mathbf{w}\right) \mathbf{F}_{1}\left(\mathbf{u}_{d}, \mathbf{w}\right) \\
& \quad=\left[\dot{\mathbf{B}}_{11}\left(\mathbf{z}_{d 0}, \mathbf{w}\right) \mathbf{t}_{d} \dot{\mathbf{B}}_{12}\left(\mathbf{z}_{d 0}, \mathbf{w}\right) \mathbf{t}_{d} \cdots \dot{\mathbf{B}}_{1 p_{1}}\left(\mathbf{z}_{d 0}, \mathbf{w}\right) \mathbf{t}_{d}\right] \\
& \quad \cdot \mathbf{F}_{1}\left(\mathbf{u}_{d}, \mathbf{w}\right)+\mathbf{B}\left(\mathbf{z}_{d 0}, \mathbf{w}\right) \mathbf{H}_{1}\left(\mathbf{u}_{d}, \mathbf{w}\right) \Longrightarrow \\
& \mathbf{C}_{1}\left(\mathbf{t}_{d}, \mathbf{z}_{d 0}, \mathbf{w}\right) \mathbf{F}_{1}\left(\mathbf{u}_{d}, \mathbf{w}\right)=\mathbf{B}\left(\mathbf{z}_{d 0}, \mathbf{w}\right) \mathbf{H}_{1}\left(\mathbf{u}_{d}, \mathbf{w}\right) \Longrightarrow \\
& \mathbf{F}_{1}\left(\mathbf{u}_{d}, \mathbf{w}\right)=\left(\mathbf{C}_{1}\left(\mathbf{t}_{d}, \mathbf{z}_{d 0}, \mathbf{w}\right)\right)^{-1} \mathbf{B}\left(\mathbf{z}_{d 0}, \mathbf{w}\right) \mathbf{H}_{1}\left(\mathbf{u}_{d}, \mathbf{w}\right), \\
& \mathbf{A}_{1}\left(\mathbf{z}_{d 0}, \mathbf{w}\right) \mathbf{F}_{2}\left(\mathbf{u}_{d}, \mathbf{w}\right)+\mathbf{A}_{2}\left(\mathbf{z}_{d 0}, \mathbf{w}\right) \\
& \quad=\left[\dot{\mathbf{B}}_{11}\left(\mathbf{z}_{d 0}, \mathbf{w}\right) \mathbf{t}_{d} \dot{\mathbf{B}}_{12}\left(\mathbf{z}_{d 0}, \mathbf{w}\right) \mathbf{t}_{d} \cdots \dot{\mathbf{B}}_{1 p_{1}}\left(\mathbf{z}_{d 0}, \mathbf{w}\right) \mathbf{t}_{d}\right] \\
& \quad \cdot \mathbf{F}_{2}\left(\mathbf{u}_{d}, \mathbf{w}\right) \\
& \quad+\left[\dot{\mathbf{B}}_{21}\left(\mathbf{z}_{d 0}, \mathbf{w}\right) \mathbf{t}_{d} \dot{\mathbf{B}}_{22}\left(\mathbf{z}_{d 0}, \mathbf{w}\right) \mathbf{t}_{d} \cdots \dot{\mathbf{B}}_{2 p_{3}}\left(\mathbf{z}_{d 0}, \mathbf{w}\right) \mathbf{t}_{d}\right] \\
& \quad+\mathbf{B}\left(\mathbf{z}_{d 0}, \mathbf{w}\right) \mathbf{H}_{2}\left(\mathbf{u}_{d}, \mathbf{w}\right) \Longrightarrow \\
& \mathbf{C}_{1}\left(\mathbf{t}_{d}, \mathbf{z}_{d 0}, \mathbf{w}\right) \mathbf{F}_{2}\left(\mathbf{u}_{d}, \mathbf{w}\right)+\mathbf{C}_{2}\left(\mathbf{t}_{d}, \mathbf{z}_{d 0}, \mathbf{w}\right)=\mathbf{B}\left(\mathbf{z}_{d 0}, \mathbf{w}\right) \\
& \quad \cdot \mathbf{H}_{2}\left(\mathbf{u}_{d}, \mathbf{w}\right) \Longrightarrow \\
& \mathbf{F}_{2}\left(\mathbf{u}_{d}, \mathbf{w}\right)=\left(\mathbf{C}_{1}\left(\mathbf{t}_{d}, \mathbf{z}_{d 0}, \mathbf{w}\right)\right)^{-1} \\
& \quad \cdot\left(\mathbf{B}\left(\mathbf{z}_{d 0}, \mathbf{w}\right) \mathbf{H}_{2}\left(\mathbf{u}_{d}, \mathbf{w}\right)-\mathbf{C}_{2}\left(\mathbf{t}_{d}, \mathbf{z}_{d 0}, \mathbf{w}\right)\right),
\end{aligned}
$$

where

$$
\begin{aligned}
\mathbf{C}_{1}\left(\mathbf{t}_{d}, \mathbf{z}_{d 0}, \mathbf{w}\right)=\mathbf{A}_{1}\left(\mathbf{z}_{d 0}, \mathbf{w}\right) \\
\quad-\left[\begin{array}{llll}
\dot{\mathbf{B}}_{11}\left(\mathbf{z}_{d 0}, \mathbf{w}\right) \mathbf{t}_{d} & \dot{\mathbf{B}}_{12}\left(\mathbf{z}_{d 0}, \mathbf{w}\right) \mathbf{t}_{d} & \cdots & \dot{\mathbf{B}}_{1 p_{1}}\left(\mathbf{z}_{d 0}, \mathbf{w}\right) \mathbf{t}_{d}
\end{array}\right] \\
\quad \in \mathbf{R}^{p_{1} \times p_{1}}, \\
\mathbf{C}_{2}\left(\mathbf{t}_{d}, \mathbf{z}_{d 0}, \mathbf{w}\right)=\mathbf{A}_{2}\left(\mathbf{z}_{d 0}, \mathbf{w}\right) \\
\quad-\left[\begin{array}{llll}
\dot{\mathbf{B}}_{21}\left(\mathbf{z}_{d 0}, \mathbf{w}\right) \mathbf{t}_{d} & \dot{\mathbf{B}}_{22}\left(\mathbf{z}_{d 0}, \mathbf{w}\right) \mathbf{t}_{d} & \cdots & \dot{\mathbf{B}}_{2 p_{3}}\left(\mathbf{z}_{d 0}, \mathbf{w}\right) \mathbf{t}_{d}
\end{array}\right] \\
\quad \in \mathbf{R}^{p_{1} \times p_{3}}
\end{aligned}
$$

$$
(1 \leq d \leq D) \text {. }
$$


in which

$$
\begin{aligned}
\mathbf{A}_{1}\left(\mathbf{z}_{d 0}, \mathbf{w}\right) & =\frac{\partial \mathbf{a}\left(\mathbf{z}_{d 0}, \mathbf{w}\right)}{\partial \mathbf{z}_{d 0}^{T}} \in \mathbf{R}^{p_{1} \times p_{1}}, \\
\mathbf{A}_{2}\left(\mathbf{z}_{d 0}, \mathbf{w}\right) & =\frac{\partial \mathbf{a}\left(\mathbf{z}_{d 0}, \mathbf{w}\right)}{\partial \mathbf{w}^{T}} \in \mathbf{R}^{p_{1} \times p_{3}} \\
\dot{\mathbf{B}}_{1 j}\left(\mathbf{z}_{d 0}, \mathbf{w}\right) & =\frac{\partial \mathbf{B}\left(\mathbf{z}_{d 0}, \mathbf{w}\right)}{\partial\left\langle\mathbf{z}_{d 0}\right\rangle_{j}} \in \mathbf{R}^{p_{1} \times\left(p_{2}+p_{4}\right)}
\end{aligned}
$$

$\left(1 \leq j \leq p_{1}\right)$

$$
\dot{\mathbf{B}}_{2 j}\left(\mathbf{z}_{d 0}, \mathbf{w}\right)=\frac{\partial \mathbf{B}\left(\mathbf{z}_{d 0}, \mathbf{w}\right)}{\partial\langle\mathbf{w}\rangle_{j}} \in \mathbf{R}^{p_{1} \times\left(p_{2}+p_{4}\right)}
$$

From (11), (13), (53), and (54), it can be verified that

$$
\begin{aligned}
& \widetilde{\mathbf{C}}_{1}\left(\widetilde{\mathbf{t}}, \widetilde{\mathbf{z}}_{0}, \mathbf{w}\right)=\operatorname{blkdiag}\left[\mathbf{C}_{1}\left(\mathbf{t}_{1}, \mathbf{z}_{10}, \mathbf{w}\right) \mathbf{C}_{1}\left(\mathbf{t}_{2}, \mathbf{z}_{20}, \mathbf{w}\right) \cdots \mathbf{C}_{1}\left(\mathbf{t}_{D}, \mathbf{z}_{D 0}, \mathbf{w}\right)\right] \\
& \widetilde{\mathbf{C}}_{2}\left(\widetilde{\mathbf{t}}, \widetilde{\mathbf{z}}_{0}, \mathbf{w}\right)=\left[\left(\mathbf{C}_{2}\left(\mathbf{t}_{1}, \mathbf{z}_{10}, \mathbf{w}\right)\right)^{T}\left(\mathbf{C}_{2}\left(\mathbf{t}_{2}, \mathbf{z}_{20}, \mathbf{w}\right)\right)^{T} \cdots\left(\mathbf{C}_{2}\left(\mathbf{t}_{D}, \mathbf{z}_{D 0}, \mathbf{w}\right)\right)^{T}\right]^{T} .
\end{aligned}
$$

Combining the second equality in (7), the first equality in (38), the first equality in (45), and the first equality in (55) and (51) yields

$$
\widetilde{\mathbf{F}}_{1}(\widetilde{\mathbf{u}}, \mathbf{w})=\left(\widetilde{\mathbf{C}}_{1}\left(\widetilde{\mathbf{t}}, \widetilde{\mathbf{z}}_{0}, \mathbf{w}\right)\right)^{-1} \widetilde{\mathbf{B}}\left(\widetilde{\mathbf{z}}_{0}, \mathbf{w}\right) \widetilde{\mathbf{H}}_{1}(\widetilde{\mathbf{u}}, \mathbf{w}) .
$$

Putting the second equality in (7), the second equality in (38), the second equality in (45), and the second equality in (55) and (52) produces

$$
\begin{aligned}
\widetilde{\mathbf{F}}_{2}(\widetilde{\mathbf{u}}, \mathbf{w})= & \left(\widetilde{\mathbf{C}}_{1}\left(\widetilde{\mathbf{t}}, \widetilde{\mathbf{z}}_{0}, \mathbf{w}\right)\right)^{-1} \\
& \cdot\left(\widetilde{\mathbf{B}}\left(\widetilde{\mathbf{z}}_{0}, \mathbf{w}\right) \widetilde{\mathbf{H}}_{2}(\widetilde{\mathbf{u}}, \mathbf{w})-\widetilde{\mathbf{C}}_{2}\left(\widetilde{\mathbf{t}}, \widetilde{\mathbf{z}}_{0}, \mathbf{w}\right)\right) .
\end{aligned}
$$

The substitution of (56) and (57) into (49) leads to

$$
\begin{aligned}
& \mathbf{P}_{1}=\left(\widetilde{\mathbf{F}}_{1}(\widetilde{\mathbf{u}}, \mathbf{w})\right)^{T} \widetilde{\mathbf{N}}^{-1} \widetilde{\mathbf{F}}_{1}(\widetilde{\mathbf{u}}, \mathbf{w}) \\
& \mathbf{P}_{2}=\left(\widetilde{\mathbf{F}}_{1}(\widetilde{\mathbf{u}}, \mathbf{w})\right)^{T} \widetilde{\mathbf{N}}^{-1} \widetilde{\mathbf{F}}_{2}(\widetilde{\mathbf{u}}, \mathbf{w}) \\
& \mathbf{P}_{3}=\left(\widetilde{\mathbf{F}}_{2}(\widetilde{\mathbf{u}}, \mathbf{w})\right)^{T} \widetilde{\mathbf{N}}^{-1} \widetilde{\mathbf{F}}_{2}(\widetilde{\mathbf{u}}, \mathbf{w})+\mathbf{M}^{-1}
\end{aligned}
$$

which combined with (44) and (48) completes the proof.

Remark 7. It can be concluded from Proposition 6 that the proposed CTLS solution is able to achieve the CRB accuracy at moderate noise level before the thresholding effect due to nonlinear nature of the estimation problem occurs. Moreover, it is worth stressing that different from the existing theoretical analysis in the literature, the proof described above is independent on the specific measurement type used. In other words, it can be considered as a more general analysis framework, which is suitable for many different location measurements. Furthermore, the experiment results in Section 7 show that the proposed method can tolerate higher noise level compared to the other location methods.

Remark 8. It is known that the performance of TLS estimator can be dependent on how the origin of the coordinate system is selected, as shown in [37]. It is obvious that such dependence also exists for the proposed CTLS method.
Indeed, it is a valuable research topic and is worthy of further investigation. However, it is not a trivial task because the mathematical analysis is rather elaborate. Hence, we can consider it as an open problem, which will be focused on in our future study.

\section{Two Localization Examples}

In this section, two localization scenarios are discussed in order to show how to exploit the proposed CTLS method to locate multiple disjoint sources and improve the sensor positions simultaneously.

6.1. Sources Localization Using TDOA and GROA Measurements. Assume that there are $D$ static and disjoint sources to be localized using a wireless location system that contains $K$ stationary sensors. The unknown source positions are denoted by $\mathbf{u}_{d}=\left[\begin{array}{lll}x_{t, d} & y_{t, d} & z_{t, d}\end{array}\right]^{T}(1 \leq d \leq D)$. The exact sensor position is represented by $\mathbf{w}_{k}=\left[\begin{array}{lll}x_{o, k} & y_{o, k} & z_{o, k}\end{array}\right]^{T} \quad(1 \leq$ $k \leq K)$, and then the system parameter is given by $\mathbf{w}=\left[\begin{array}{llll}\mathbf{w}_{1}^{T} & \mathbf{w}_{2}^{T} & \cdots & \mathbf{w}_{K}^{T}\end{array}\right]^{T}$. As previously assumed, the system parameter available for processing, denoted by $\mathbf{v}$, has random errors. The TDOA and GROA measurements with respect to the reference sensor, say sensor 1 , are exacted from the received signals. Note that the TDOA and GROA measurements are equivalent to the range difference and range ratio measurements, respectively. As a consequence, the corresponding observation equations are given by

$$
\begin{aligned}
& \rho_{d, k}=\left\|\mathbf{u}_{d}-\mathbf{w}_{k}\right\|_{2}-\left\|\mathbf{u}_{d}-\mathbf{w}_{1}\right\|_{2} \\
& r_{d, k}=\frac{\left\|\mathbf{u}_{d}-\mathbf{w}_{k}\right\|_{2}}{\left\|\mathbf{u}_{d}-\mathbf{w}_{1}\right\|_{2}}
\end{aligned}
$$

$$
\left(\begin{array}{l}
2 \leq k \leq K \\
1 \leq d \leq D
\end{array}\right)
$$

Let us define the following vectors:

$$
\begin{aligned}
& \boldsymbol{\rho}_{d}=\left[\begin{array}{llll}
\rho_{d, 2} & \rho_{d, 3} & \cdots & \rho_{d, K}
\end{array}\right]^{T}, \\
& \mathbf{r}_{d}=\left[\begin{array}{llll}
r_{d, 2} & r_{d, 3} & \cdots & r_{d, K}
\end{array}\right]^{T} .
\end{aligned}
$$


Then, the noiseless measurement vector related to the $d$ th source is given by

$$
\mathbf{z}_{d 0}=\left[\begin{array}{ll}
\boldsymbol{\rho}_{d}^{T} & \mathbf{r}_{d}^{T}
\end{array}\right]^{T}=\mathbf{f}\left(\mathbf{u}_{d}, \mathbf{w}\right) \in \mathbf{R}^{2(K-1) \times 1}
$$

$$
(1 \leq d \leq D) .
$$

The collection of all the measurements forms the $2(K-1) D \times 1$ vector as follows:

$$
\begin{aligned}
\widetilde{\mathbf{z}}_{0} & =\left[\begin{array}{llll}
\mathbf{z}_{10}^{T} & \mathbf{z}_{20}^{T} & \cdots & \mathbf{z}_{D 0}^{T}
\end{array}\right]^{T} \\
& =\left[\begin{array}{llll}
\left(\mathbf{f}\left(\mathbf{u}_{1}, \mathbf{w}\right)\right)^{T} & \left(\mathbf{f}\left(\mathbf{u}_{2}, \mathbf{w}\right)\right)^{T} & \cdots & \left(\mathbf{f}\left(\mathbf{u}_{D}, \mathbf{w}\right)\right)^{T}
\end{array}\right]^{T} \\
& =\widetilde{\mathbf{f}}(\widetilde{\mathbf{u}}, \mathbf{w}) \in \mathbf{R}^{2(K-1) D \times 1} .
\end{aligned}
$$

In order to employ the proposed CTLS method for source localization, we must transform the nonlinear equations in (59) into the pseudo-linear ones by introducing some auxiliary variables. First, it follows from the first equation in (59) that

$$
\begin{aligned}
& \rho_{d, k}=\left\|\mathbf{u}_{d}-\mathbf{w}_{k}\right\|_{2}-\left\|\mathbf{u}_{d}-\mathbf{w}_{1}\right\|_{2} \Longrightarrow \\
& \left(\rho_{d, k}+\left\|\mathbf{u}_{d}-\mathbf{w}_{1}\right\|_{2}\right)^{2}=\left\|\mathbf{u}_{d}-\mathbf{w}_{1}+\mathbf{w}_{1}-\mathbf{w}_{k}\right\|_{2}^{2} \Longrightarrow \\
& 2\left(\mathbf{w}_{1}-\mathbf{w}_{k}\right)^{T}\left(\mathbf{u}_{d}-\mathbf{w}_{1}\right)-2 \rho_{d, k} \cdot\left\|\mathbf{u}_{d}-\mathbf{w}_{1}\right\|_{2} \\
& \quad=\rho_{d, k}^{2}-\left\|\mathbf{w}_{1}-\mathbf{w}_{k}\right\|_{2}^{2} \Longrightarrow \\
& \left(\mathbf{b}_{1 k}\left(\mathbf{z}_{d 0}, \mathbf{w}\right)\right)^{T} \cdot\left[\begin{array}{c}
\mathbf{u}_{d}-\mathbf{w}_{1} \\
\left\|\mathbf{u}_{d}-\mathbf{w}_{1}\right\|_{2}
\end{array}\right]=a_{1 k}\left(\mathbf{z}_{d 0}, \mathbf{w}\right)
\end{aligned}
$$

$$
(2 \leq k \leq K ; 1 \leq d \leq D),
$$

where

$$
\begin{aligned}
& \mathbf{b}_{1 k}\left(\mathbf{z}_{d 0}, \mathbf{w}\right)=\left[2\left(\mathbf{w}_{1}-\mathbf{w}_{k}\right)^{T} \mid-2 \rho_{d, k}\right]^{T} \\
& a_{1 k}\left(\mathbf{z}_{d 0}, \mathbf{w}\right)=\rho_{d, k}^{2}-\left\|\mathbf{w}_{1}-\mathbf{w}_{k}\right\|_{2}^{2} .
\end{aligned}
$$

At the same time, we also obtain from (59) that

$$
\begin{aligned}
& r_{d, k}=\frac{\left\|\mathbf{u}_{d}-\mathbf{w}_{k}\right\|_{2}}{\left\|\mathbf{u}_{d}-\mathbf{w}_{1}\right\|_{2}} \Longrightarrow \\
& \left\|\mathbf{u}_{d}-\mathbf{w}_{k}\right\|_{2}=r_{d, k} \cdot\left\|\mathbf{u}_{d}-\mathbf{w}_{1}\right\|_{2} \Longrightarrow \\
& \left(r_{d, k}-1\right) \cdot\left\|\mathbf{u}_{d}-\mathbf{w}_{1}\right\|_{2}=\left\|\mathbf{u}_{d}-\mathbf{w}_{k}\right\|_{2}-\left\|\mathbf{u}_{d}-\mathbf{w}_{1}\right\|_{2} \\
& \quad=\rho_{d, k} \Longrightarrow \\
& \left(\mathbf{b}_{2 k}\left(\mathbf{z}_{d 0}, \mathbf{w}\right)\right)^{T} \cdot\left[\begin{array}{c}
\mathbf{u}_{d}-\mathbf{w}_{1} \\
\left\|\mathbf{u}_{d}-\mathbf{w}_{1}\right\|_{2}
\end{array}\right]=a_{2 k}\left(\mathbf{z}_{d 0}, \mathbf{w}\right) \\
& \quad(2 \leq k \leq K ; 1 \leq d \leq D),
\end{aligned}
$$

where

$$
\begin{aligned}
& \mathbf{b}_{2 k}\left(\mathbf{z}_{d 0}, \mathbf{w}\right)=\left[\mathbf{O}_{1 \times 3} \mid r_{d, k}-1\right]^{T} \\
& a_{2 k}\left(\mathbf{z}_{d 0}, \mathbf{w}\right)=\rho_{d, k} .
\end{aligned}
$$

Combining (63)-(66) yields the following pseudo-linear vector equation:

$$
\begin{array}{r}
\mathbf{a}\left(\mathbf{z}_{d 0}, \mathbf{w}\right)=\mathbf{B}\left(\mathbf{z}_{d 0}, \mathbf{w}\right) \mathbf{t}_{d}=\mathbf{B}\left(\mathbf{z}_{d 0}, \mathbf{w}\right) \mathbf{h}\left(\mathbf{u}_{d}, \mathbf{w}\right) \\
(1 \leq d \leq D),
\end{array}
$$

where

$$
\begin{aligned}
\mathbf{a}\left(\mathbf{z}_{d 0}, \mathbf{w}\right) & =\left[\begin{array}{ll}
\left(\mathbf{a}_{1}\left(\mathbf{z}_{d 0}, \mathbf{w}\right)\right)^{T} & \left(\mathbf{a}_{2}\left(\mathbf{z}_{d 0}, \mathbf{w}\right)\right)^{T}
\end{array}\right]^{T} \\
\mathbf{B}\left(\mathbf{z}_{d 0}, \mathbf{w}\right) & =\left[\begin{array}{ll}
\left(\mathbf{B}_{1}\left(\mathbf{z}_{d 0}, \mathbf{w}\right)\right)^{T} & \left(\mathbf{B}_{2}\left(\mathbf{z}_{d 0}, \mathbf{w}\right)\right)^{T}
\end{array}\right]^{T} \\
\mathbf{t}_{d} & =\mathbf{h}\left(\mathbf{u}_{d}, \mathbf{w}\right)=\left[\begin{array}{c}
\mathbf{u}_{d}-\mathbf{w}_{1} \\
\left\|\mathbf{u}_{d}-\mathbf{w}_{1}\right\|_{2}
\end{array}\right]=\left[\begin{array}{c}
\mathbf{u}_{d}-\mathbf{J w} \\
\mathbf{s}\left(\mathbf{u}_{d}, \mathbf{w}\right)
\end{array}\right]
\end{aligned}
$$

in which

$$
\begin{gathered}
\mathbf{B}_{j}\left(\mathbf{z}_{d 0}, \mathbf{w}\right)=\left[\begin{array}{c}
\mathbf{b}_{j 2}^{T}\left(\mathbf{z}_{d 0}, \mathbf{w}\right) \\
\mathbf{b}_{j 3}^{T}\left(\mathbf{z}_{d 0}, \mathbf{w}\right) \\
\vdots \\
\mathbf{b}_{j K}^{T}\left(\mathbf{z}_{d 0}, \mathbf{w}\right)
\end{array}\right], \\
\mathbf{a}_{j}\left(\mathbf{z}_{d 0}, \mathbf{w}\right)=\left[\begin{array}{c}
a_{j 2}\left(\mathbf{z}_{d 0}, \mathbf{w}\right) \\
a_{j 3}\left(\mathbf{z}_{d 0}, \mathbf{w}\right) \\
\vdots \\
a_{j K}\left(\mathbf{z}_{d 0}, \mathbf{w}\right)
\end{array}\right]
\end{gathered}
$$

$(1 \leq j \leq 2)$

$$
\begin{aligned}
\mathbf{s}\left(\mathbf{u}_{d}, \mathbf{w}\right) & =\left\|\mathbf{u}_{d}-\mathbf{w}_{1}\right\|_{2}, \\
\mathbf{J} & =\left[\begin{array}{ll}
\mathbf{I}_{3} & \mathbf{O}_{3 \times 3(K-1)}
\end{array}\right] .
\end{aligned}
$$

Collecting all the $D$ vector equations in (67) gives

$$
\widetilde{\mathbf{a}}\left(\widetilde{\mathbf{z}}_{0}, \mathbf{w}\right)=\widetilde{\mathbf{B}}\left(\widetilde{\mathbf{z}}_{0}, \mathbf{w}\right) \widetilde{\mathbf{t}}=\widetilde{\mathbf{B}}\left(\widetilde{\mathbf{z}}_{0}, \mathbf{w}\right) \widetilde{\mathbf{h}}(\widetilde{\mathbf{u}}, \mathbf{w}),
$$

where

$$
\begin{aligned}
& \widetilde{\mathbf{a}}\left(\widetilde{\mathbf{z}}_{0}, \mathbf{w}\right)
\end{aligned}
$$

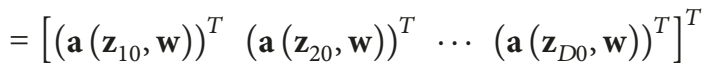

$$
\begin{aligned}
& \widetilde{\mathbf{B}}\left(\widetilde{\mathbf{z}}_{0}, \mathbf{w}\right) \\
& =\operatorname{blkdiag}\left[\mathbf{B}\left(\mathbf{z}_{10}, \mathbf{w}\right) \quad \mathbf{B}\left(\mathbf{z}_{20}, \mathbf{w}\right) \cdots \mathbf{B}\left(\mathbf{z}_{D 0}, \mathbf{w}\right)\right] \\
& \widetilde{\mathbf{t}}=\widetilde{\mathbf{h}}(\widetilde{\mathbf{u}}, \mathbf{w})=\left[\begin{array}{llll}
\mathbf{t}_{1}^{T} & \mathbf{t}_{2}^{T} & \cdots & \mathbf{t}_{D}^{T}
\end{array}\right]^{T} \\
& =\left[\begin{array}{llll}
\left(\mathbf{h}\left(\mathbf{u}_{1}, \mathbf{w}\right)\right)^{T} & \left(\mathbf{h}\left(\mathbf{u}_{2}, \mathbf{w}\right)\right)^{T} & \ldots & \left(\mathbf{h}\left(\mathbf{u}_{D}, \mathbf{w}\right)\right)^{T}
\end{array}\right]^{T}
\end{aligned}
$$

$\widetilde{\mathbf{z}}_{0}=\left[\begin{array}{llll}\mathbf{z}_{10}^{T} & \mathbf{z}_{20}^{T} & \cdots & \mathbf{z}_{D 0}^{T}\end{array}\right]^{T}$,

$\widetilde{\mathbf{u}}=\left[\begin{array}{llll}\mathbf{u}_{1}^{T} & \mathbf{u}_{2}^{T} & \cdots & \mathbf{u}_{D}^{T}\end{array}\right]^{T}$. 
According to the discussions in the previous sections and in Appendix A, we also need to derive the expressions for $\mathbf{C}_{1}\left(\mathbf{t}_{d}, \mathbf{z}_{d 0}, \mathbf{w}\right), \quad \mathbf{C}_{2}\left(\mathbf{t}_{d}, \mathbf{z}_{d 0}, \mathbf{w}\right), \mathbf{H}_{1}\left(\mathbf{u}_{d}, \mathbf{w}\right)$, $\mathbf{H}_{2}\left(\mathbf{u}_{d}, \mathbf{w}\right), \partial \operatorname{vec}\left(\left(\mathbf{S}_{1}\left(\mathbf{u}_{d}, \mathbf{w}\right)\right)^{T}\right) / \partial \mathbf{u}_{d}^{T}, \partial \operatorname{vec}\left(\left(\mathbf{S}_{2}\left(\mathbf{u}_{d}, \mathbf{w}\right)\right)^{T}\right) / \partial \mathbf{u}_{d}^{T}$, $\partial \operatorname{vec}\left(\left(\mathbf{S}_{1}\left(\mathbf{u}_{d}, \mathbf{w}\right)\right)^{T}\right) / \partial \mathbf{w}^{T}$, and $\partial \operatorname{vec}\left(\left(\mathbf{S}_{2}\left(\mathbf{u}_{d}, \mathbf{w}\right)\right)^{T}\right) / \partial \mathbf{w}^{T}$. The detailed derivations of them are provided in Appendix C. At this point, the CTLS localization method using TDOA and GROA measurements can be summarized as follows.

Step 1. Compute $\widetilde{\mathbf{B}}(\widetilde{\mathbf{z}}, \mathbf{v})$ and $\widetilde{\mathbf{a}}(\widetilde{\mathbf{z}}, \mathbf{v})$ according to (63)-(71), and determine the initial values by WLS or TLS methods.

Step 2. Compute $\widetilde{\mathbf{C}}_{1}(\widetilde{\mathbf{t}}, \widetilde{\mathbf{z}}, \mathbf{v})$ and $\widetilde{\mathbf{C}}_{2}(\widetilde{t}, \widetilde{\mathbf{z}}, \mathbf{v})$ using (55) as well as the expressions for $\mathbf{C}_{1}\left(\mathbf{t}_{d}, \mathbf{z}_{d 0}, \mathbf{w}\right)$ and $\mathbf{C}_{2}\left(\mathbf{t}_{d}, \mathbf{z}_{d 0}, \mathbf{w}\right)$, and calculate $\mathbf{G}(\widetilde{\mathbf{u}}, \mathbf{w})$ according to (18) and (24).

Step 3. Compute $\overline{\widetilde{\mathbf{H}}}_{1}(\widetilde{\mathbf{u}}, \mathbf{w})$ and $\overline{\widetilde{\mathbf{H}}}_{2}(\widetilde{\mathbf{u}}, \mathbf{w})$ from (38), (39), (A.3), and (A.4) as well as the expressions for $\mathbf{H}_{1}\left(\mathbf{u}_{d}, \mathbf{w}\right)$ and $\mathbf{H}_{2}\left(\mathbf{u}_{d}, \mathbf{w}\right)$, and calculate $\mathbf{Z}_{1}$ and $\mathbf{Z}_{2}$ using (A.1) and (A.2).

Step 4. Compute $\mathbf{Z}_{3}, \mathbf{Z}_{4}, \mathbf{Z}_{5}$, and $\mathbf{Z}_{6}$ from (A.5)-(A.13) as well as the expressions for $\partial \operatorname{vec}\left(\left(\mathbf{S}_{1}\left(\mathbf{u}_{d}, \mathbf{w}\right)\right)^{T}\right) / \partial \mathbf{u}_{d}^{T}, \partial \operatorname{vec}\left(\left(\mathbf{S}_{2}\left(\mathbf{u}_{d}\right.\right.\right.$, $\left.\mathbf{w}))^{T}\right) / \partial \mathbf{u}_{d}^{T}$, $\partial \operatorname{vec}\left(\left(\mathbf{S}_{1}\left(\mathbf{u}_{d}, \mathbf{w}\right)\right)^{T}\right) / \partial \mathbf{w}^{T}$, and $\partial \operatorname{vec}\left(\left(\mathbf{S}_{2}\left(\mathbf{u}_{d}, \mathbf{w}\right)\right)^{T}\right) /$ $\partial \mathbf{w}^{T}$.

Step 5. Compute $\mathbf{Z}_{7}$ and $\mathbf{Z}_{8}$ according to (A.14)-(A.21).

Step 6. Compute gradient $\varphi(\widetilde{\mathbf{u}}, \mathbf{w})$ from (25)-(26), and compute Hessian matrix $\Psi(\widetilde{\mathbf{u}}, \mathbf{w})$ according to (27)-(32).

Step 7. If the predefined convergence criterion is satisfied, then terminate; otherwise update the unknowns using (33) and continue with Step 2.

\subsection{Sources Localization Using TOA and FOA Measurements.}

It is assumed that there are $D$ moving and disjoint sources to be located by a wireless location system that is composed of $K$ moving sensors. The position and velocity of the $d$ th source are denoted by $\mathbf{u}_{d, p}=\left[\begin{array}{lll}x_{t, d} & y_{t, d} & z_{t, d}\end{array}\right]^{T}$ and $\mathbf{u}_{d, v}=\left[\begin{array}{lll}\dot{x}_{t, d} & \dot{y}_{t, d} & \dot{z}_{t, d}\end{array}\right]^{T}$, respectively. The location parameter of source $d$ is defined by $\mathbf{u}_{d}=\left[\begin{array}{ll}\mathbf{u}_{d, p}^{T} & \mathbf{u}_{d, v}^{T}\end{array}\right]^{T}$. The true position and velocity of the $k$ th sensor are represented by $\mathbf{w}_{k, p}=$ $\left[\begin{array}{lll}x_{o, k} & y_{o, k} & z_{o, k}\end{array}\right]^{T}$ and $\mathbf{w}_{k, v}=\left[\begin{array}{lll}\dot{x}_{o, k} & \dot{y}_{o, k} & \dot{z}_{o, k}\end{array}\right]^{T}$, respectively. Define $\mathbf{w}_{k}=\left[\begin{array}{ll}\mathbf{w}_{k, p}^{T} & \mathbf{w}_{k, v}^{T}\end{array}\right]^{T}$, and then the system parameter is given by $\mathbf{w}=\left[\begin{array}{llll}\mathbf{w}_{1}^{T} & \mathbf{w}_{2}^{T} & \cdots & \mathbf{w}_{K}^{T}\end{array}\right]^{T}$. Note that the vector $\mathbf{w}$ is not known exactly and only the noisy version of it, denoted by $\mathbf{v}$, is available in practice. Additionally, the TOA and FOA measurements are obtained from the observed signals. Since the TOA and FOA measurements are equivalent to the range and range rate measurements, respectively, the relevant observation equations can be expressed by

$$
\begin{aligned}
& \mu_{d, k}=\left\|\mathbf{u}_{d, p}-\mathbf{w}_{k, p}\right\|_{2}, \\
& \dot{\mu}_{d, k}=\frac{\left(\mathbf{u}_{d, p}-\mathbf{w}_{k, p}\right)^{T}\left(\mathbf{u}_{d, v}-\mathbf{w}_{k, v}\right)}{\left\|\mathbf{u}_{d, p}-\mathbf{w}_{k, p}\right\|_{2}}
\end{aligned}
$$

$$
(1 \leq k \leq K) \text {. }
$$

Define the following vectors:

$$
\begin{aligned}
& \boldsymbol{\mu}_{d}=\left[\begin{array}{llll}
\mu_{d, 1} & \mu_{d, 2} & \cdots & \mu_{d, K}
\end{array}\right]^{T}, \\
& \dot{\boldsymbol{\mu}}_{d}=\left[\begin{array}{llll}
\dot{\mu}_{d, 1} & \dot{\mu}_{d, 2} & \cdots & \dot{\mu}_{d, K}
\end{array}\right]^{T} .
\end{aligned}
$$

Then, the noiseless measurement vector associated with the $d$ th source is given by

$$
\mathbf{z}_{d 0}=\left[\begin{array}{ll}
\boldsymbol{\mu}_{d}^{T} & \dot{\boldsymbol{\mu}}_{d}^{T}
\end{array}\right]^{T}=\mathbf{f}\left(\mathbf{u}_{d}, \mathbf{w}\right) \in \mathbf{R}^{2 K \times 1} \quad(1 \leq d \leq D) .
$$

Gathering all the measurements in a $2 K D \times 1$ vector leads to

$$
\begin{aligned}
\widetilde{\mathbf{z}}_{0} & =\left[\begin{array}{llll}
\mathbf{z}_{10}^{T} & \mathbf{z}_{20}^{T} & \cdots & \mathbf{z}_{D 0}^{T}
\end{array}\right]^{T} \\
& =\left[\begin{array}{llll}
\left(\mathbf{f}\left(\mathbf{u}_{1}, \mathbf{w}\right)\right)^{T} & \left(\mathbf{f}\left(\mathbf{u}_{2}, \mathbf{w}\right)\right)^{T} & \cdots & \left(\mathbf{f}\left(\mathbf{u}_{D}, \mathbf{w}\right)\right)^{T}
\end{array}\right]^{T} \\
& =\widetilde{\mathbf{f}}(\widetilde{\mathbf{u}}, \mathbf{w}) \in \mathbf{R}^{2 K D \times 1} .
\end{aligned}
$$

To make use of the presented CTLS localization method, we must convert the nonlinear equations in (72) into the pseudo-linear ones by introducing some instrumental variables. From the first equation in (72), we have

$$
\begin{array}{r}
\mu_{d, k}=\left\|\mathbf{u}_{d, p}-\mathbf{w}_{k, p}\right\|_{2} \Longrightarrow \\
2 \mathbf{w}_{k, p}^{T} \mathbf{u}_{d, p}-\left\|\mathbf{u}_{d, p}\right\|_{2}^{2}=\left\|\mathbf{w}_{k, p}\right\|_{2}^{2}-\mu_{d, k}^{2} \Longrightarrow \\
\left(\mathbf{b}_{1 k}\left(\mathbf{z}_{d 0}, \mathbf{w}\right)\right)^{T} \cdot\left[\begin{array}{c}
\mathbf{u}_{d, p} \\
\mathbf{u}_{d, v} \\
\left\|\mathbf{u}_{d, p}\right\|_{2}^{2} \\
\mathbf{u}_{d, v}^{T} \mathbf{u}_{d, p}
\end{array}\right]
\end{array}
$$

where

$$
\begin{aligned}
& \mathbf{b}_{1 k}\left(\mathbf{z}_{d 0}, \mathbf{w}\right)=\left[2 \mathbf{w}_{k, p}^{T}\left|\mathbf{O}_{1 \times 3}\right|-1 \mid 0\right]^{T}, \\
& a_{1 k}\left(\mathbf{z}_{d 0}, \mathbf{w}\right)=\left\|\mathbf{w}_{k, p}\right\|_{2}^{2}-\mu_{d, k}^{2} .
\end{aligned}
$$

Taking the time derivation of the second equation in (76) leads to

$$
\begin{aligned}
& 2 \mathbf{w}_{k, p}^{T} \mathbf{u}_{d, p}-\left\|\mathbf{u}_{d, p}\right\|_{2}^{2}=\left\|\mathbf{w}_{k, p}\right\|_{2}^{2}-\mu_{d, k}^{2} \Longrightarrow \\
& \mathbf{w}_{k, v}^{T} \mathbf{u}_{d, p}+\mathbf{w}_{k, p}^{T} \mathbf{u}_{d, v}-\mathbf{u}_{d, v}^{T} \mathbf{u}_{d, p}=\mathbf{w}_{k, v}^{T} \mathbf{w}_{k, p}-\mu_{d, k} \dot{\mu}_{d, k} \Longrightarrow \\
& \left(\mathbf{b}_{2 k}\left(\mathbf{z}_{d 0}, \mathbf{w}\right)\right)^{T} \cdot\left[\begin{array}{c}
\mathbf{u}_{d, p} \\
\mathbf{u}_{d, v} \\
\left\|\mathbf{u}_{d, p}\right\|_{2}^{2} \\
\mathbf{u}_{d, v}^{T} \mathbf{u}_{d, p}
\end{array}\right]=a_{2 k}\left(\mathbf{z}_{d 0}, \mathbf{w}\right)
\end{aligned}
$$


where

$$
\begin{aligned}
& \mathbf{b}_{2 k}\left(\mathbf{z}_{d 0}, \mathbf{w}\right)=\left[\mathbf{w}_{k, v}^{T}\left|\mathbf{w}_{k, p}^{T}\right| 0 \mid-1\right]^{T}, \\
& a_{2 k}\left(\mathbf{z}_{d 0}, \mathbf{w}\right)=\mathbf{w}_{k, v}^{T} \mathbf{w}_{k, p}-\mu_{d, k} \dot{\mu}_{d, k} .
\end{aligned}
$$

Putting (76)-(79) together gives the following pseudo-linear vector equation:

$$
\begin{array}{r}
\mathbf{a}\left(\mathbf{z}_{d 0}, \mathbf{w}\right)=\mathbf{B}\left(\mathbf{z}_{d 0}, \mathbf{w}\right) \mathbf{t}_{d}=\mathbf{B}\left(\mathbf{z}_{d 0}, \mathbf{w}\right) \mathbf{h}\left(\mathbf{u}_{d}, \mathbf{w}\right) \\
(1 \leq d \leq D),
\end{array}
$$

where

$$
\begin{aligned}
\mathbf{a}\left(\mathbf{z}_{d 0}, \mathbf{w}\right) & =\left[\begin{array}{ll}
\left(\mathbf{a}_{1}\left(\mathbf{z}_{d 0}, \mathbf{w}\right)\right)^{T} & \left(\mathbf{a}_{2}\left(\mathbf{z}_{d 0}, \mathbf{w}\right)\right)^{T}
\end{array}\right]^{T} \\
\mathbf{B}\left(\mathbf{z}_{d 0}, \mathbf{w}\right) & =\left[\begin{array}{ll}
\left(\mathbf{B}_{1}\left(\mathbf{z}_{d 0}, \mathbf{w}\right)\right)^{T} & \left(\mathbf{B}_{2}\left(\mathbf{z}_{d 0}, \mathbf{w}\right)\right)^{T}
\end{array}\right]^{T} \\
\mathbf{t}_{d} & =\mathbf{h}\left(\mathbf{u}_{d}, \mathbf{w}\right)=\left[\begin{array}{c}
\mathbf{u}_{d} \\
\left\|\mathbf{u}_{d, p}\right\|_{2}^{2} \\
\mathbf{u}_{d, v}^{T} \mathbf{u}_{d, p}
\end{array}\right]=\left[\begin{array}{c}
\mathbf{u}_{d}-\mathbf{J w} \\
\mathbf{s}\left(\mathbf{u}_{d}, \mathbf{w}\right)
\end{array}\right]
\end{aligned}
$$

in which

$$
\begin{aligned}
\mathbf{B}_{j}\left(\mathbf{z}_{d 0}, \mathbf{w}\right) & =\left[\begin{array}{c}
\mathbf{b}_{j 1}^{T}\left(\mathbf{z}_{d 0}, \mathbf{w}\right) \\
\mathbf{b}_{j 2}^{T}\left(\mathbf{z}_{d 0}, \mathbf{w}\right) \\
\vdots \\
\mathbf{b}_{j K}^{T}\left(\mathbf{z}_{d 0}, \mathbf{w}\right)
\end{array}\right], \\
\mathbf{a}_{j}\left(\mathbf{z}_{d 0}, \mathbf{w}\right) & =\left[\begin{array}{c}
a_{j 1}\left(\mathbf{z}_{d 0}, \mathbf{w}\right) \\
a_{j 2}\left(\mathbf{z}_{d 0}, \mathbf{w}\right) \\
\vdots \\
a_{j K}\left(\mathbf{z}_{d 0}, \mathbf{w}\right)
\end{array}\right], \\
\mathbf{s}\left(\mathbf{u}_{d}, \mathbf{w}\right) & =\left[\begin{array}{c}
\left\|\mathbf{u}_{d, p}\right\|_{2}^{2} \\
\mathbf{u}_{d, v}^{T} \mathbf{u}_{d, p}
\end{array}\right], \\
\mathbf{J} & =\mathbf{O}_{6 \times 6 K} .
\end{aligned}
$$

It can be readily seen from (81) and (82) that neither $\mathbf{h}\left(\mathbf{u}_{d}, \mathbf{w}\right)$ nor $\mathbf{s}\left(\mathbf{u}_{d}, \mathbf{w}\right)$ is dependent on $\mathbf{w}$ for the localization scenario under discussion. This leads to a reduction of the computation load. Putting (80) together for $d=1,2, \ldots, D$ gives

$$
\widetilde{\mathbf{a}}\left(\widetilde{\mathbf{z}}_{0}, \mathbf{w}\right)=\widetilde{\mathbf{B}}\left(\widetilde{\mathbf{z}}_{0}, \mathbf{w}\right) \widetilde{\mathbf{t}}=\widetilde{\mathbf{B}}\left(\widetilde{\mathbf{z}}_{0}, \mathbf{w}\right) \widetilde{\mathbf{h}}(\widetilde{\mathbf{u}}, \mathbf{w}),
$$

where

$$
\begin{aligned}
& \widetilde{\mathbf{a}}\left(\widetilde{\mathbf{z}}_{0}, \mathbf{w}\right) \\
& \quad=\left[\begin{array}{llll}
\left(\mathbf{a}\left(\mathbf{z}_{10}, \mathbf{w}\right)\right)^{T} & \left(\mathbf{a}\left(\mathbf{z}_{20}, \mathbf{w}\right)\right)^{T} & \ldots & \left(\mathbf{a}\left(\mathbf{z}_{D 0}, \mathbf{w}\right)\right)^{T}
\end{array}\right]^{T},
\end{aligned}
$$

TABLE 1: Nominal positions of sensors.

\begin{tabular}{lccc}
\hline Sensor number $k$ & $x_{o, k}(\mathrm{~m})$ & $y_{o, k}(\mathrm{~m})$ & $z_{o, k}(\mathrm{~m})$ \\
\hline$(1)$ & 1800 & -2000 & 1200 \\
$(2)$ & -1400 & 1800 & 1600 \\
$(3)$ & 1700 & -1400 & -1500 \\
$(4)$ & -1100 & 1300 & -1800 \\
$(5)$ & 1800 & 1500 & 2100 \\
$(6)$ & -1900 & -1200 & -1700 \\
\hline
\end{tabular}

$$
\begin{aligned}
\widetilde{\mathbf{B}} & \left(\widetilde{\mathbf{z}}_{0}, \mathbf{w}\right) \\
& =\operatorname{blkdiag}\left[\begin{array}{llll}
\mathbf{B}\left(\mathbf{z}_{10}, \mathbf{w}\right) & \mathbf{B}\left(\mathbf{z}_{20}, \mathbf{w}\right) & \cdots & \mathbf{B}\left(\mathbf{z}_{D 0}, \mathbf{w}\right)
\end{array}\right], \\
\widetilde{\mathbf{t}} & =\widetilde{\mathbf{h}}(\widetilde{\mathbf{u}}, \mathbf{w})=\left[\begin{array}{llll}
\mathbf{t}_{1}^{T} & \mathbf{t}_{2}^{T} & \cdots & \mathbf{t}_{D}^{T}
\end{array}\right]^{T} \\
& =\left[\begin{array}{llll}
\left(\mathbf{h}\left(\mathbf{u}_{1}, \mathbf{w}\right)\right)^{T} & \left(\mathbf{h}\left(\mathbf{u}_{2}, \mathbf{w}\right)\right)^{T} & \cdots & \left(\mathbf{h}\left(\mathbf{u}_{D}, \mathbf{w}\right)\right)^{T}
\end{array}\right]^{T}, \\
\widetilde{\mathbf{z}}_{0} & =\left[\begin{array}{llll}
\mathbf{z}_{10}^{T} & \mathbf{z}_{20}^{T} & \cdots & \mathbf{z}_{D 0}^{T}
\end{array}\right]^{T}, \\
\widetilde{\mathbf{u}} & =\left[\begin{array}{llll}
\mathbf{u}_{1}^{T} & \mathbf{u}_{2}^{T} & \cdots & \mathbf{u}_{D}^{T}
\end{array}\right]^{T} .
\end{aligned}
$$

Based on the discussions in the previous sections and in Appendix $A$, we also need to derive the expressions for $\mathbf{C}_{1}\left(\mathbf{t}_{d}, \mathbf{z}_{d 0}, \mathbf{w}\right), \mathbf{C}_{2}\left(\mathbf{t}_{d}, \mathbf{z}_{d 0}, \mathbf{w}\right), \mathbf{H}_{1}\left(\mathbf{u}_{d}, \mathbf{w}\right)$, $\mathbf{H}_{2}\left(\mathbf{u}_{d}, \mathbf{w}\right), \partial \operatorname{vec}\left(\left(\mathbf{S}_{1}\left(\mathbf{u}_{d}, \mathbf{w}\right)\right)^{T}\right) / \partial \mathbf{u}_{d}^{T}, \partial \operatorname{vec}\left(\left(\mathbf{S}_{2}\left(\mathbf{u}_{d}, \mathbf{w}\right)\right)^{T}\right) / \partial \mathbf{u}_{d}^{T}$, $\partial \operatorname{vec}\left(\left(\mathbf{S}_{1}\left(\mathbf{u}_{d}, \mathbf{w}\right)\right)^{T}\right) / \partial \mathbf{w}^{T}$, and $\partial \operatorname{vec}\left(\left(\mathbf{S}_{2}\left(\mathbf{u}_{d}, \mathbf{w}\right)\right)^{T}\right) / \partial \mathbf{w}^{T}$. The detailed derivations of them are shown in Appendix D. On the other hand, it is obvious that the algorithm described in Section 6.1 is applicable here, and we thus omit it due to limited space.

\section{Simulations Results}

In this section, some computer simulations are reported to illustrate the behavior of the presented method. The rootmean-square-error (RMSE) and norm of bias are chosen as performance metrics. All the simulation results are averaged over 5000 independent noise realizations. The proposed solution is implemented using the procedure described in Section 6 . The initial value of this iterative algorithm is given by the WLS method, the result of which is equal to the firststep estimate of the TWLS method.

7.1. Numerical Results for TDOAs/GROAs Source Localization. In this subsection, the simulations are performed for source localization using TDOA and GROA measurements. The estimation accuracy of the proposed CTLS algorithm is compared to that of the TLS algorithm and the TWLS algorithm, as well as the corresponding CRB given by (44). Additionally, in order to show the cooperation gain resulting from joint localization for multiple sources, the CRB obtained from (44) is also compared to the CRB for the case in which the sources are located independently.

The localization scenario contains 6 sensors, and their nominal positions are given in Table 1. The 


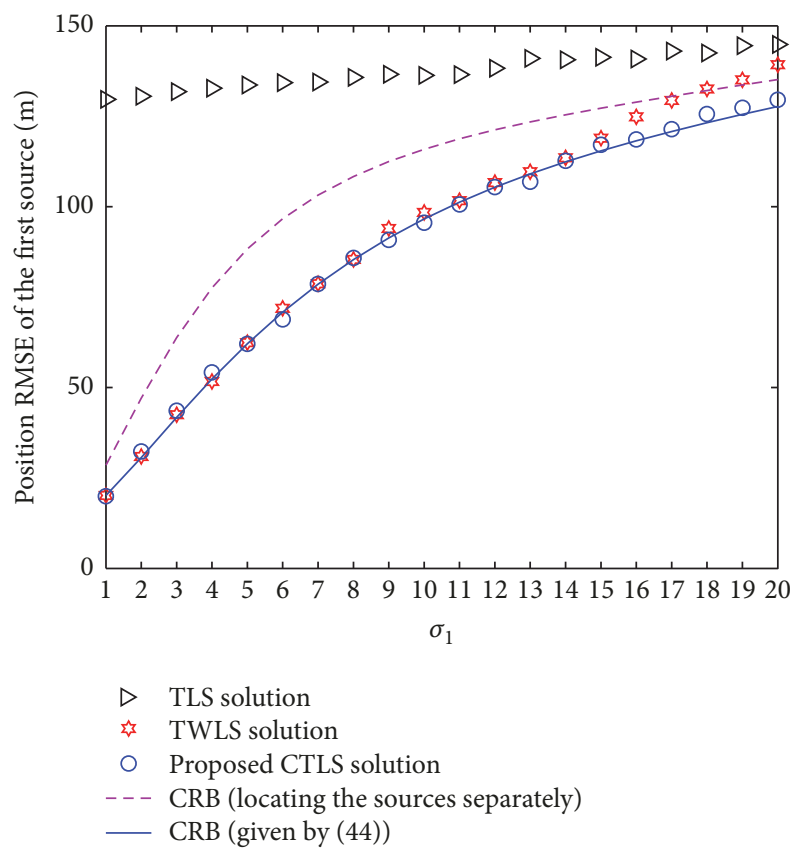

FIGURE 1: RMSE of the estimated position for the first source versus $\sigma_{1}$.

noisy sensor locations are created by adding to the true values zero-mean white Gaussian noise with covariance matrix $\mathbf{M}=\sigma_{w}^{2} \mathbf{I}_{3 K}$. There are three disjoint sources to be located, and their true positions are $\mathbf{u}_{1}=$ $\left[\begin{array}{lll}6000 & 6000 & 3000\end{array}\right]^{T}(\mathrm{~m}), \mathbf{u}_{2}=\left[\begin{array}{lll}6500 & 6500 & 3500\end{array}\right]^{T}(\mathrm{~m})$, and $\mathbf{u}_{3}=\left[\begin{array}{lll}7000 & 7000 & 4000\end{array}\right]^{T}(\mathrm{~m})$. The TDOAs and GROAs for a given source are generated by adding the zero-mean Gaussian noise to the true values. The covariance matrix is $\mathbf{N}_{d}=\operatorname{blkdiag}\left[\sigma_{\mathrm{TDOA}}^{2} \mathbf{R} \sigma_{\mathrm{GROA}}^{2} \mathbf{R}\right]$, where $\mathbf{R}$ is a $(K-1) \times(K-1)$ matrix with diagonal elements equal to 1 and all other elements 0.5 . The measurements from different sources are independent of each other. Consequently, the covariance matrix $\widetilde{\mathbf{N}}$ is block diagonal.

In the first experiment, we fix $\sigma_{w}=5$ and set $\sigma_{\mathrm{TDOA}}=$ $0.15 \sigma_{1} / c, \sigma_{\mathrm{GROA}}=0.0015 \sigma_{1}$, where $\sigma_{1}$ varies from 1 to 20 and $c$ is the signal propagation speed. Figures $1-3$, respectively, display the RMSE of position estimates for the three sources versus $\sigma_{1}$. Figure 4 plots the RMSE of the estimated receiving position as a function of $\sigma_{1}$. In the second experiment, we fix $\sigma_{\mathrm{TDOA}}=1 / c, \sigma_{\mathrm{GROA}}=0.01$ and set $\sigma_{w}=0.6 \sigma_{2}$, where $\sigma_{2}$ ranges from 1 to 20 . Figures 5-7, respectively, show the RMSE of location estimates for the three sources versus $\sigma_{2}$. Figure 8 illustrates the RMSE of the estimated receiving position as a function of $\sigma_{2}$.

It can be seen from Figures 1-8 that the proposed CTLS method can reach the CRB given by (44) under moderate noise level, which demonstrates the validity of the performance analysis in Section 5. Moreover, the proposed method achieves noticeably better accuracy than the TLS method, and it has a higher noise threshold than the TWLS method. By comparing the two kinds of CRB, we can find that the performance improvement due to joint localization

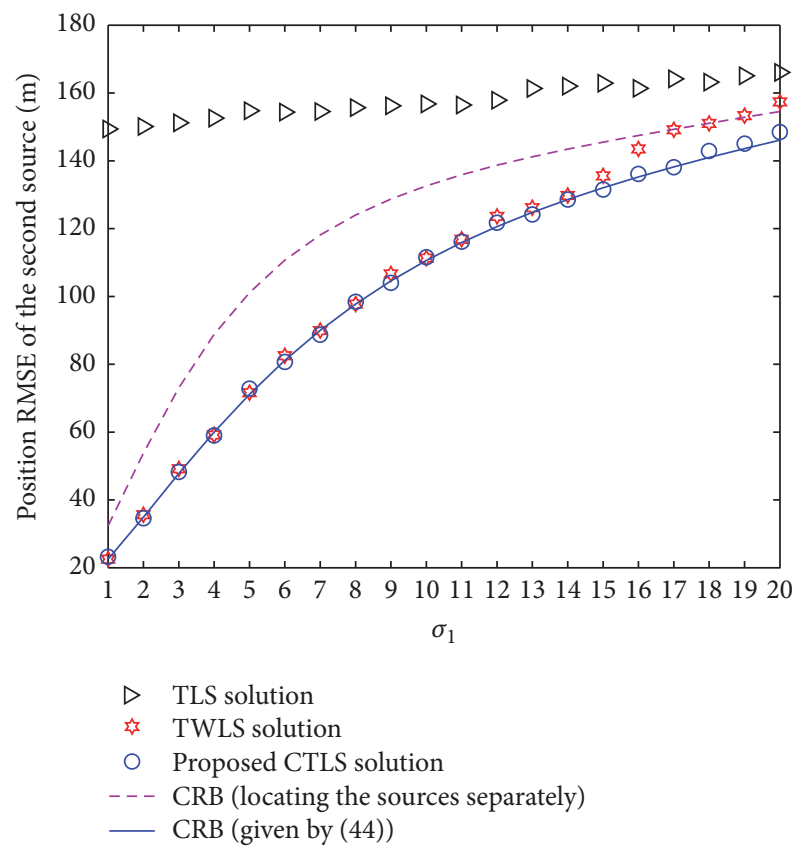

FIGURE 2: RMSE of the estimated position for the second source versus $\sigma_{1}$.

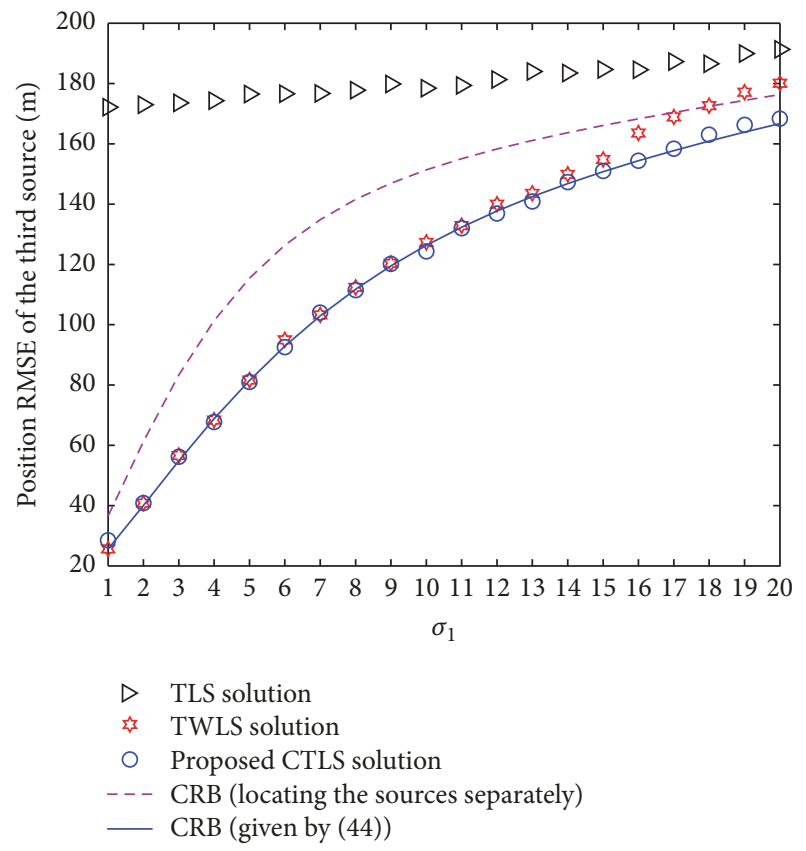

FIGURE 3: RMSE of the estimated position for the third source versus $\sigma_{1}$.

is remarkable. Moreover, it can be observed from Figures 5-7 that the cooperation gain increases with increasing $\sigma_{2}$. The reason is that as $\sigma_{2}$ increases the correlation between the measurements of distinct sources becomes larger and consequently the effect of cooperative processing can be more considerable. On the other hand, we can also find from Figures 4 and 8 that compared to the prior knowledge of the 

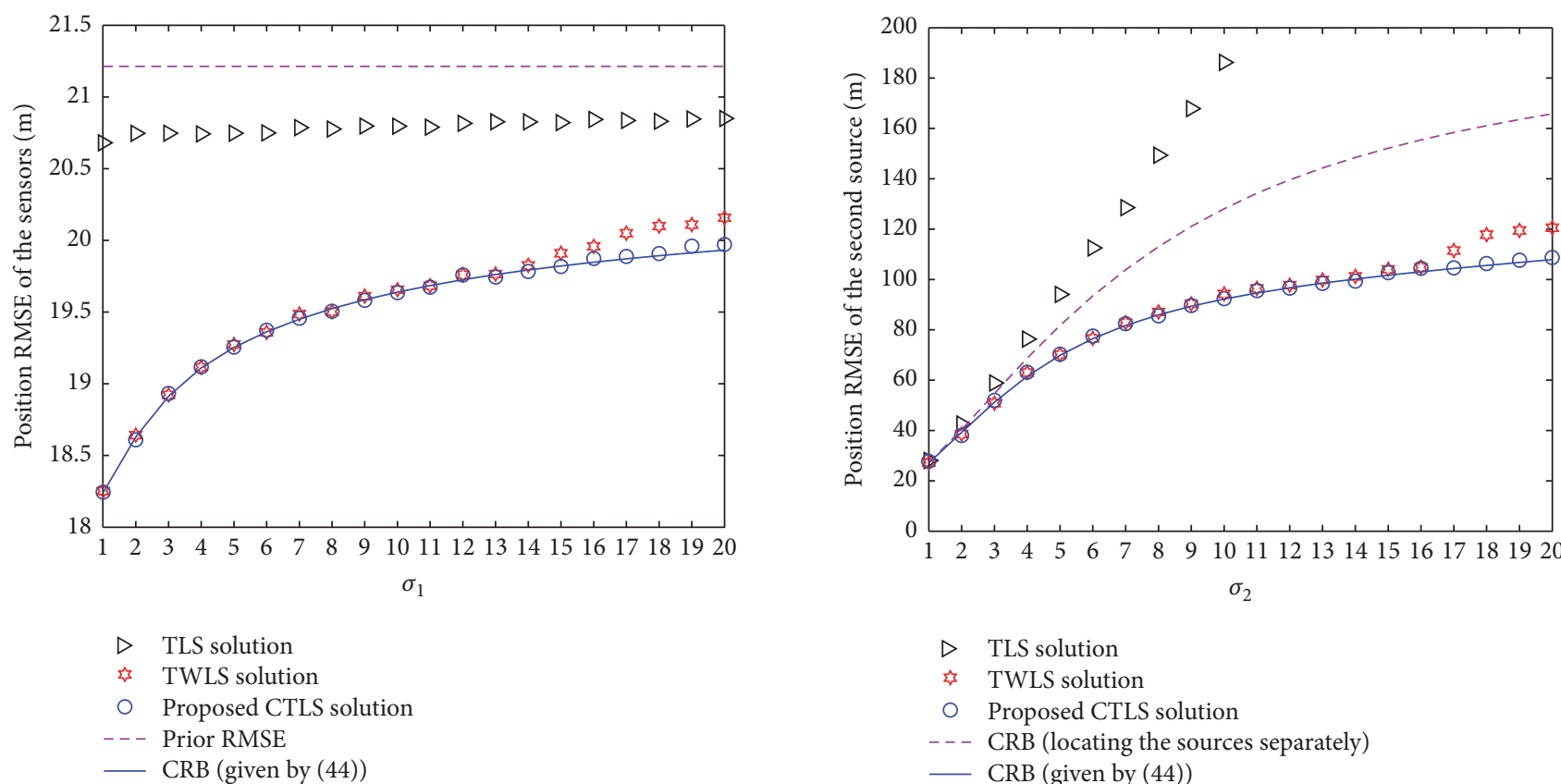

FIGURE 4: RMSE of the sensor position estimate versus $\sigma_{1}$.

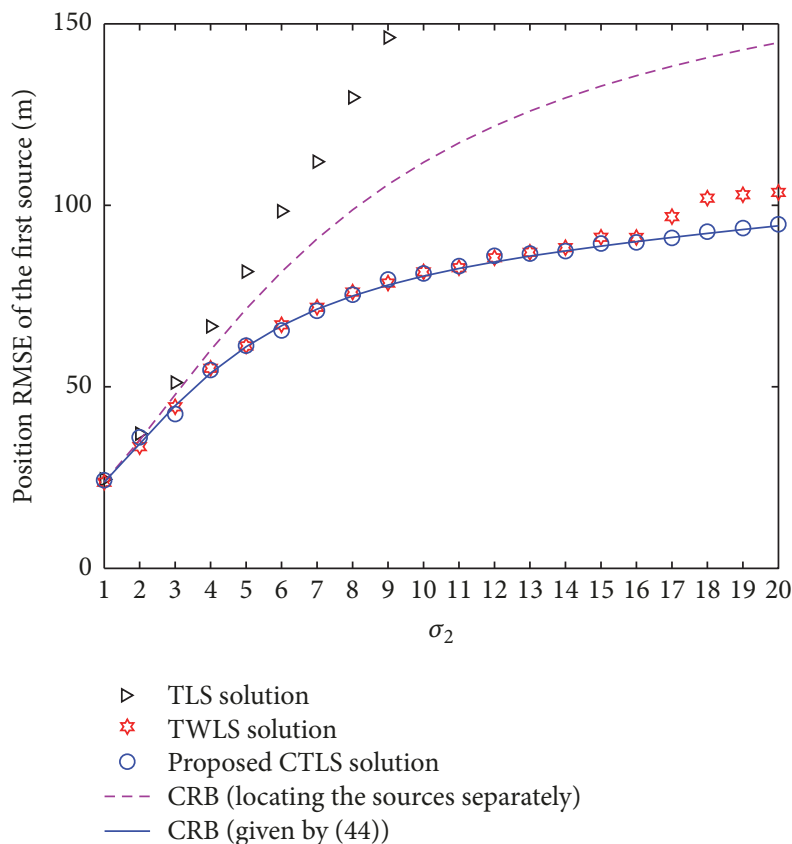

FIGURE 5: RMSE of the estimated position for the first source as a function of $\sigma_{2}$.

sensor positions, the proposed CTLS method can provide an estimate with a smaller RMSE.

In the following experiments, we compare the norm of source position bias of the proposed CTLS method with that of the TWLS method. The simulation parameters are set as the same as previously described, except that the standard deviations of noises are changed.

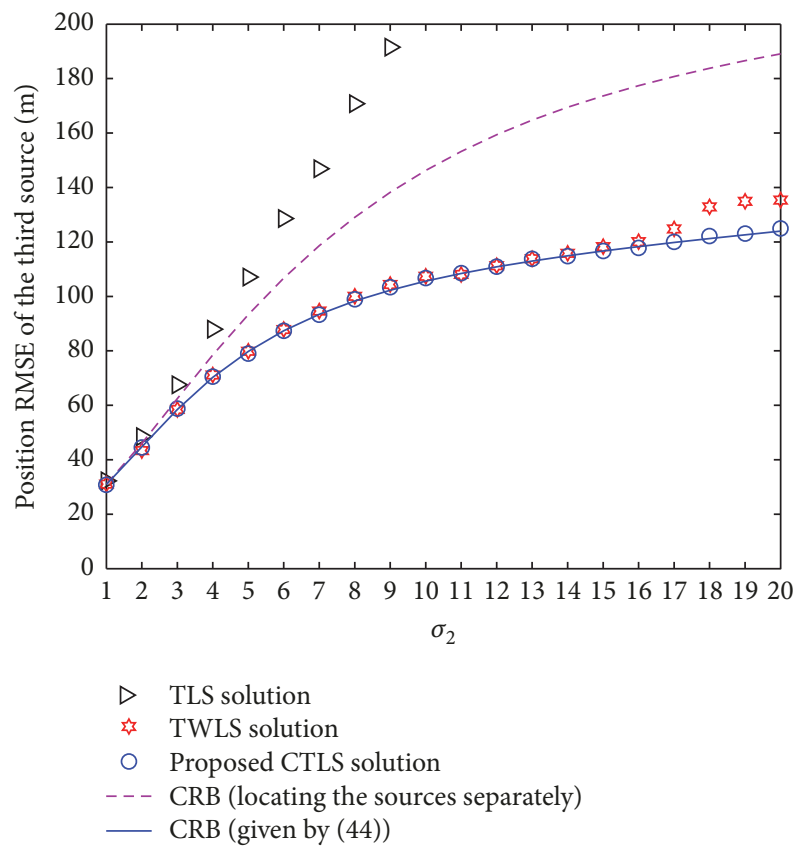

FIGURE 7: RMSE of the estimated position for the third source as a function of $\sigma_{2}$.

First, we let $\sigma_{w}=20$ and set $\sigma_{\mathrm{TDOA}}=0.3 \sigma_{1} / c, \sigma_{\mathrm{GROA}}=$ $0.003 \sigma_{1}$, where $\sigma_{1}$ varies from 1 to 20 . Figure 9 depicts the norm of source position bias for the three sources as a function of $\sigma_{1}$. Next, we choose $\sigma_{\mathrm{TDOA}}=3 / c, \sigma_{\mathrm{GROA}}=0.03$ and set $\sigma_{w}=2 \sigma_{2}$, where $\sigma_{2}$ ranges from 1 to 20 . Figure 10 plots the norm of source position bias for the three sources versus $\sigma_{2}$. 


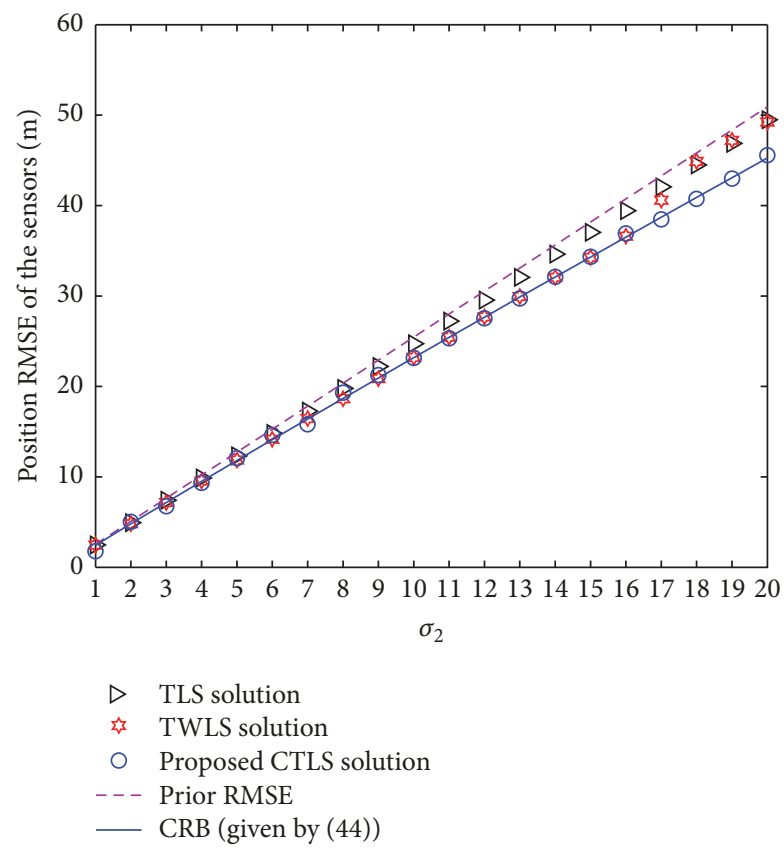

FIGURE 8: RMSE of the sensor position estimate as a function of $\sigma_{2}$.

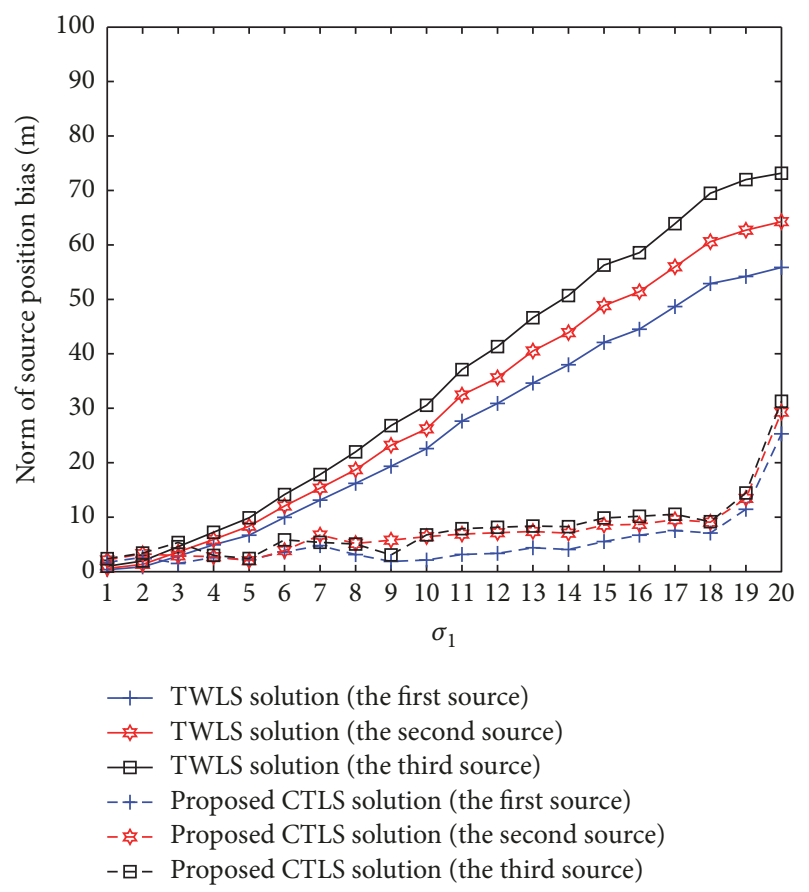

FIgURE 9: Norm of source position bias as a function of $\sigma_{1}$.

Figures 9 and 10 show that the estimation bias of the proposed CTLS method is much smaller than that of the TWLS method, especially when the noise is large. This observation is not unexpected because, as studied in [38], the TWLS method has large estimation bias at high noise level. Additionally, the proposed CTLS method can yield relatively small deviation, as expected. The reason lies in that it can remove the bias by updating the weighting matrix in the

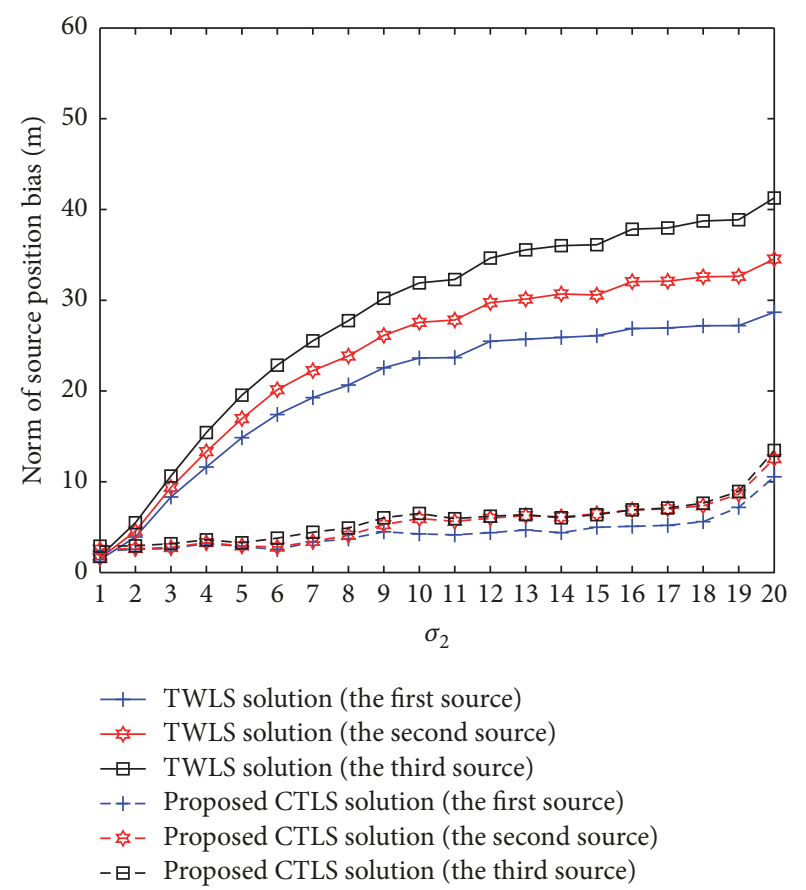

FIGURE 10: Norm of source position bias as a function of $\sigma_{2}$.

iterative process, as stated in Remark 5. On the other hand, in order to prevent the curves in Figures 9 and 10 from being messy, the estimation bias of the TLS method is not included in Figures 9 and 10. Indeed, the bias of this method is found to be considerably larger than that of the other two methods due to the fact that the TLS estimator is biased, as studied in [1].

7.2. Numerical Results for TOAs/FOAs Source Localization. In this subsection, the simulations are carried out for the localization scenario using TOA and FOA measurements. We compare the performance of the proposed CTLS algorithm with the TLS algorithm and the TS algorithm as well as the corresponding CRB computed by (44). Besides, for the purpose of showing the advantage of cooperative localization, the CRB for the case of single-source location is displayed again. On the other hand, it is noteworthy that the TS algorithm requires initial solution guess. However, good initial estimate is not easily available for this algorithm because it does not provide the pseudo-linear vector equation. For a comprehensive comparison, the TS algorithm is initialized in two ways. One chooses random value as initial guess and the other takes the true value as initial solution.

In the following simulation, an array of 6 sensors is used to locate the disjoint sources, and the nominal positions and velocities of sensors are listed in Table 2. The sensor location and velocity errors follow zeromean Gaussian distribution with covariance matrix $\mathbf{M}=\mathbf{I}_{K} \otimes \operatorname{blkdiag}\left[\begin{array}{ccc}\sigma_{w_{p}}^{2} \mathbf{I}_{3} & \sigma_{w_{v}}^{2} \mathbf{I}_{3}\end{array}\right]$. Three moving sources need to be located. Their true positions are $\mathbf{u}_{1, p}=$ $\left[\begin{array}{lll}6000 & 6000 & 3000\end{array}\right]^{T}(\mathrm{~m}), \mathbf{u}_{2, p}=\left[\begin{array}{lll}6500 & 6500 & 3500\end{array}\right]^{T}(\mathrm{~m})$, 
TABLE 2: Nominal positions and velocities of sensors.

\begin{tabular}{|c|c|c|c|c|c|c|}
\hline Sensor number $k$ & $x_{o, k}(\mathrm{~m})$ & $y_{o, k}(\mathrm{~m})$ & $z_{o, k}(\mathrm{~m})$ & $\dot{x}_{o, k}(\mathrm{~m} / \mathrm{s})$ & $\dot{y}_{o, k}(\mathrm{~m} / \mathrm{s})$ & $\dot{z}_{o, k}(\mathrm{~m} / \mathrm{s})$ \\
\hline (1) & 2000 & -2000 & 1500 & 20 & -30 & 10 \\
\hline (2) & -1400 & 1800 & 1900 & -10 & -10 & 20 \\
\hline (3) & 1400 & -1500 & -1600 & 20 & 30 & -10 \\
\hline (4) & -1300 & 1400 & -1300 & 10 & 20 & 10 \\
\hline (5) & 1600 & 1800 & 2000 & -20 & -10 & -30 \\
\hline (6) & -1700 & -1400 & -1600 & -10 & 20 & 20 \\
\hline
\end{tabular}

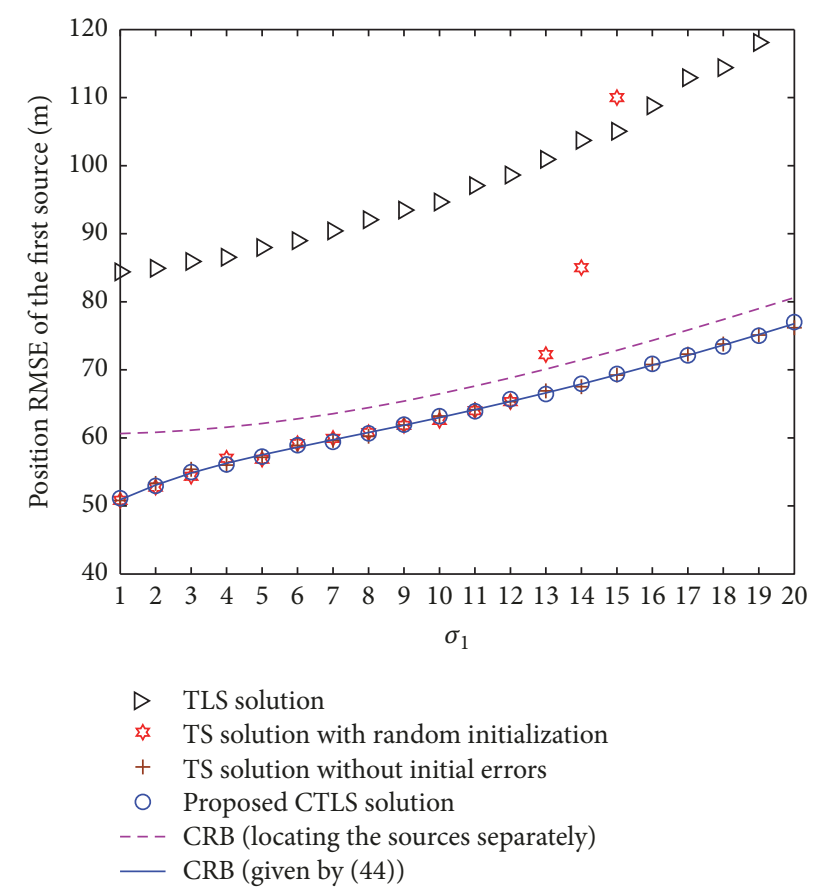

FIGURE 11: RMSE of the estimated position for the first source versus $\sigma_{1}$.

and $\mathbf{u}_{3, p}=\left[\begin{array}{lll}7000 & 7000 & 4000\end{array}\right]^{T}(\mathrm{~m})$. Their exact velocities are $\mathbf{u}_{1, v}=\left[\begin{array}{lll}10 & -20 & 20\end{array}\right]^{T}(\mathrm{~m} / \mathrm{s}), \mathbf{u}_{2, v}=\left[\begin{array}{lll}20 & 20 & 10\end{array}\right]^{T}(\mathrm{~m} / \mathrm{s})$, and $\mathbf{u}_{3, v}=\left[\begin{array}{lll}30 & 10 & -10\end{array}\right]^{T}(\mathrm{~m} / \mathrm{s})$. The TOA/FOA measurement errors for a given source are zero-mean Gaussian distributed with covariance matrix $\mathbf{N}_{d}=$ blkdiag $\left[\begin{array}{llll}\sigma_{\mathrm{TOA}}^{2} \mathbf{I}_{K} & \sigma_{\mathrm{FOA}}^{2} \mathbf{I}_{K}\end{array}\right]$. The measurements from different sources are assumed to be uncorrelated with each other, which leads to a block-diagonal structure of the covariance matrix $\widetilde{\mathbf{N}}$.

In the first experiment, we fix $\sigma_{w, p}=20, \sigma_{w, v}=0.5$ and set $\sigma_{\mathrm{TOA}}=\sigma_{1} / c, \sigma_{\mathrm{FOA}}=0.01 f_{0} \sigma_{1} / c$, where $\sigma_{1}$ is changed from 1 to 20 and $f_{0}$ is the signal carrier frequency. Figures 11-13, respectively, plot the RMSE of position estimates for the three sources versus $\sigma_{1}$. Figures 14-16, respectively, plot the RMSE of velocity estimates for the three sources versus $\sigma_{1}$. Figures 17 and 18 plot the RMSE of position and velocity estimates for the sensor as a function of $\sigma_{1}$, respectively.

In the second experiment, we fix $\sigma_{\mathrm{TOA}}=3 / \mathrm{c}, \sigma_{\mathrm{FOA}}=$ $0.01 f_{0} / c$ and set $\sigma_{w, p}=1.5 \sigma_{2}, \sigma_{w, v}=0.05 \sigma_{2}$, where $\sigma_{2}$ varies

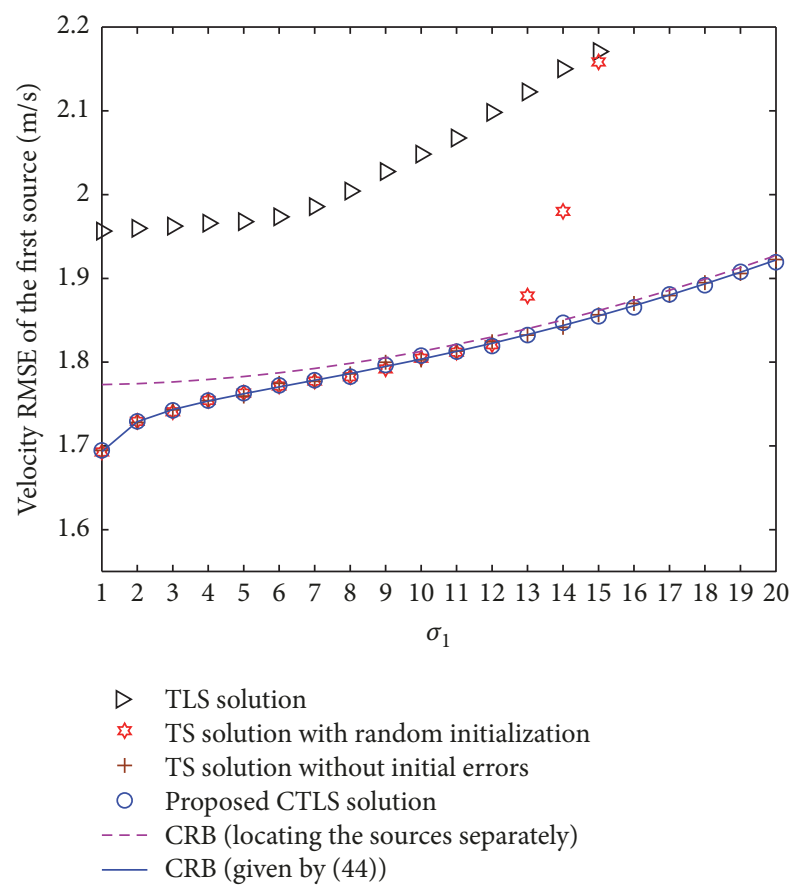

FIGURE 12: RMSE of the estimated velocity for the first source versus $\sigma_{1}$.

from 1 to 20. Figures 19-21, respectively, illustrate the RMSE of position estimates for the three sources versus $\sigma_{2}$. Figures 22-24, respectively, show the RMSE of velocity estimates for the three sources versus $\sigma_{2}$. Figures 25 and 26 plot the RMSE of position and velocity estimates for the sensor as a function of $\sigma_{2}$, respectively.

It can be observed from Figures 11-26 that the proposed CTLS solution achieves the CRB accuracy given by (44) under moderate noise level, which can corroborate the theoretical comparison between the performance of the proposed estimator and the CRB. The proposed method still outperforms the TLS method for this localization scenario. The advantage of cooperation localization is also noticeable by comparing the two kinds of CRB. Additionally, if the TS algorithm is initialized with the true value, it yields a nearly similar performance to the proposed CTLS algorithm. However, when the initial value of the TS algorithm is chosen randomly, its performance deviates from the CRB earlier compared to the CTLS algorithm. Indeed, it is hard 


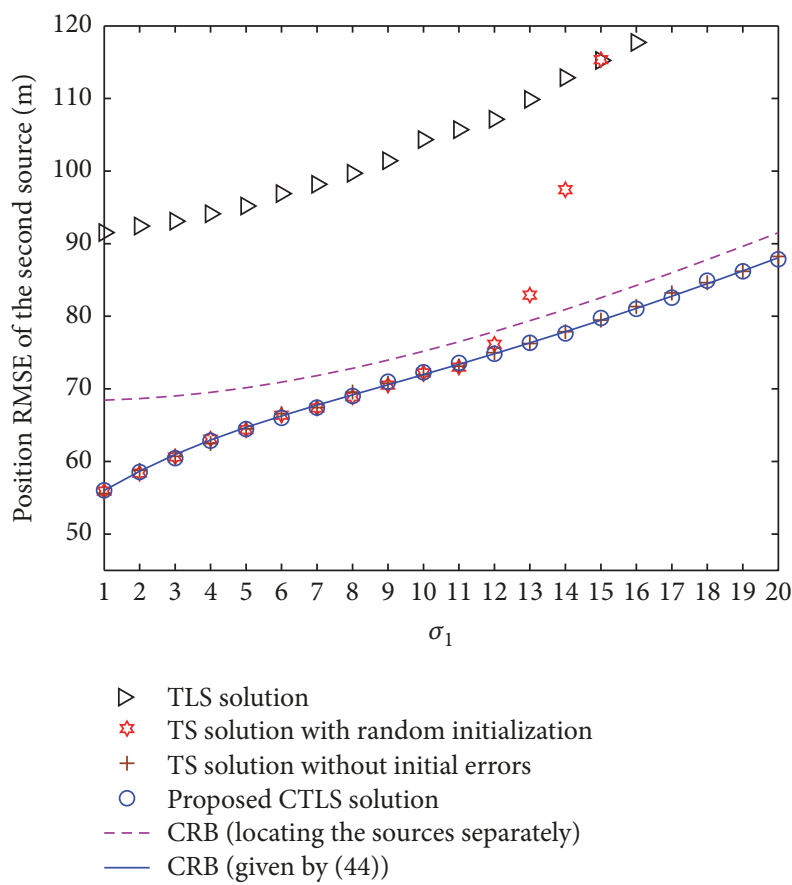

FIGURE 13: RMSE of the estimated position for the second source versus $\sigma_{1}$.

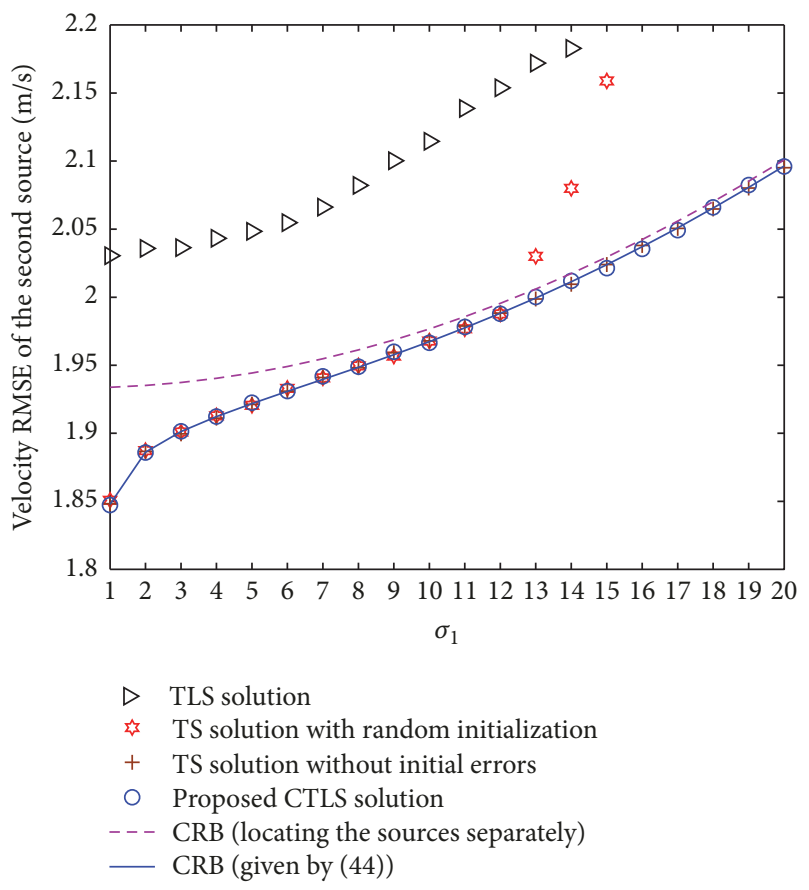

FIGURE 14: RMSE of the estimated velocity for the second source versus $\sigma_{1}$.

to get a good initial estimate for the TS algorithm because this algorithm does not yield a pseudo-linear measurement equation. On the other hand, it can also be seen from Figures $17,18,25$, and 26 that the proposed method can improve the estimation accuracy for the sensor locations in comparison to its prior position information.

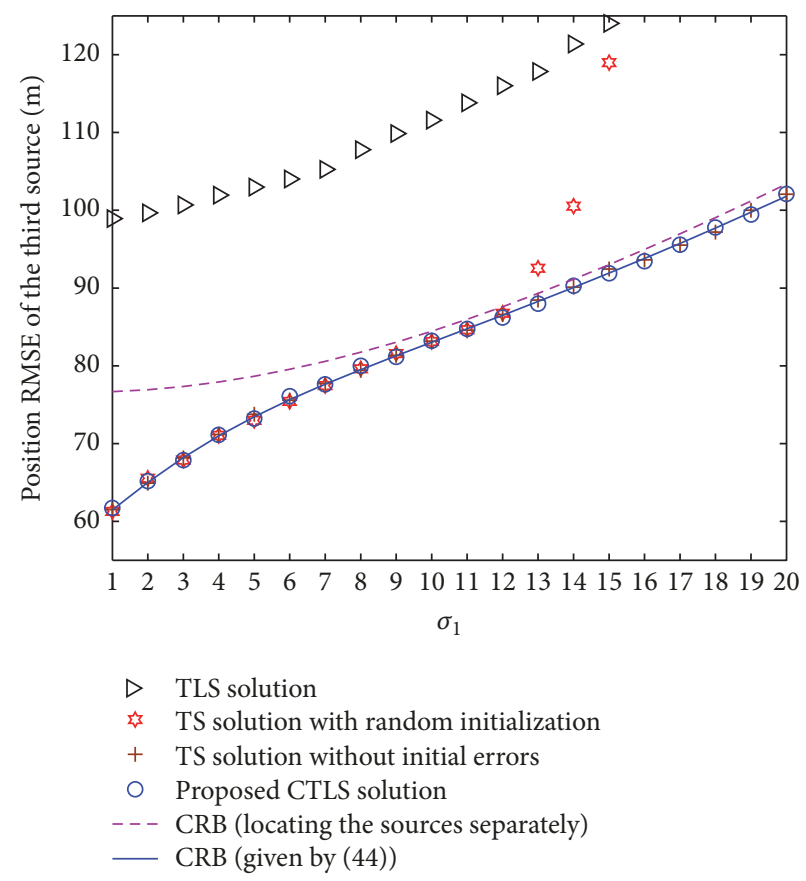

FIGURE 15: RMSE of the estimated velocity for the third source versus $\sigma_{1}$.

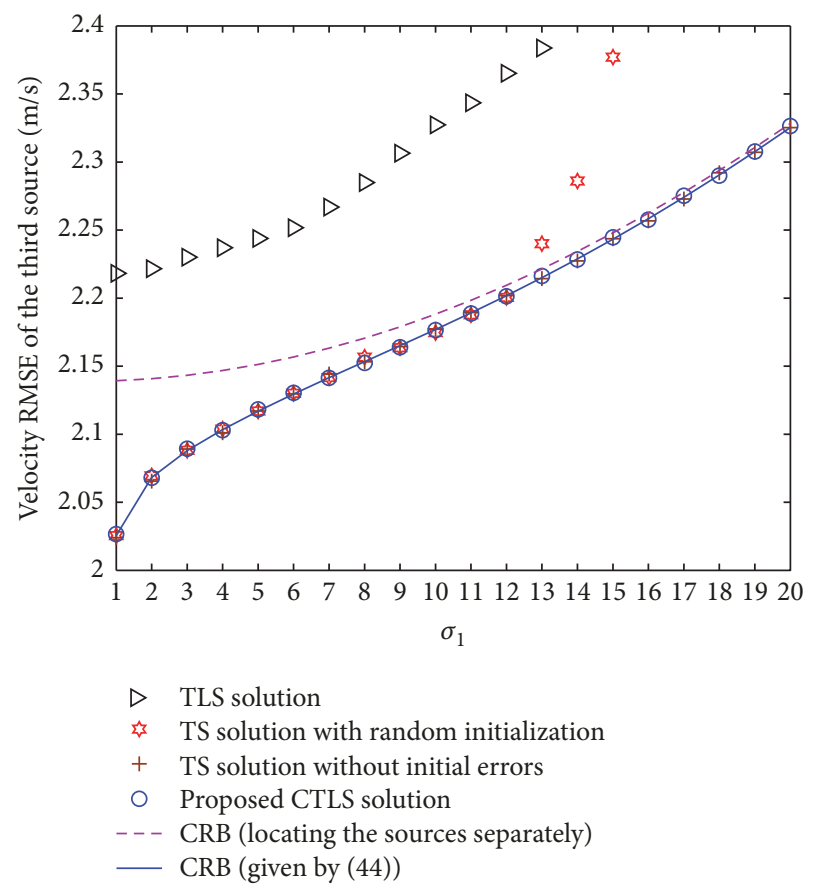

FIGURE 16: RMSE of the estimated velocity for the third source versus $\sigma_{1}$.

In the following experiments, we compare the norm of source position bias of the proposed CTLS solution with that of the TS algorithm, which is randomly initialized. The simulation parameters are assumed the same as those stated above, except that we change the standard deviations of noises. 


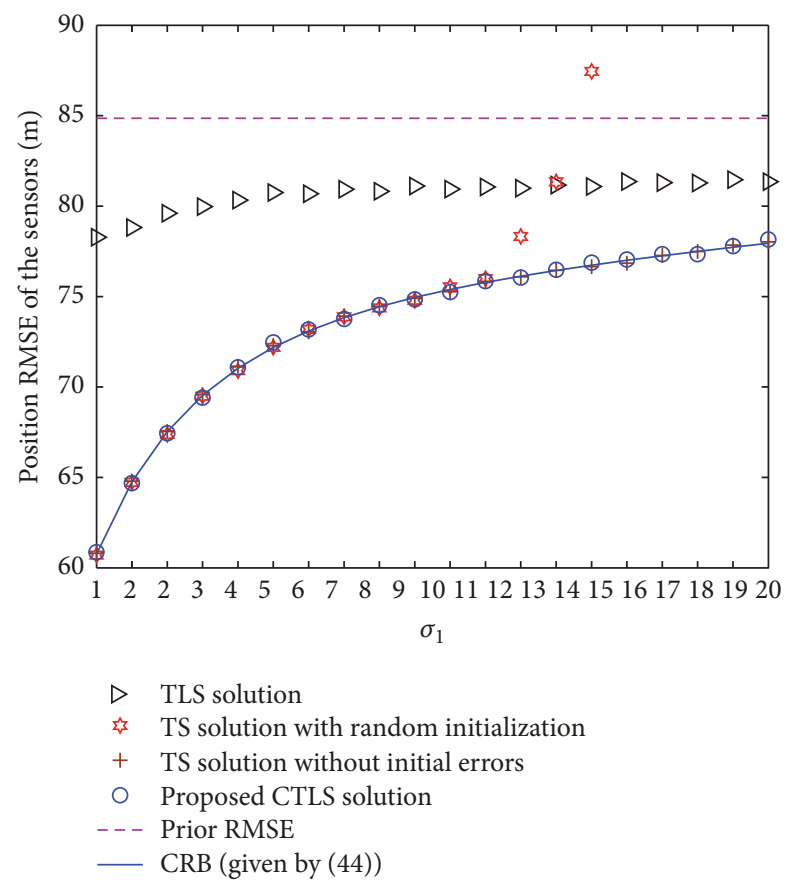

FIGURE 17: RMSE of the sensor position estimate versus $\sigma_{1}$.

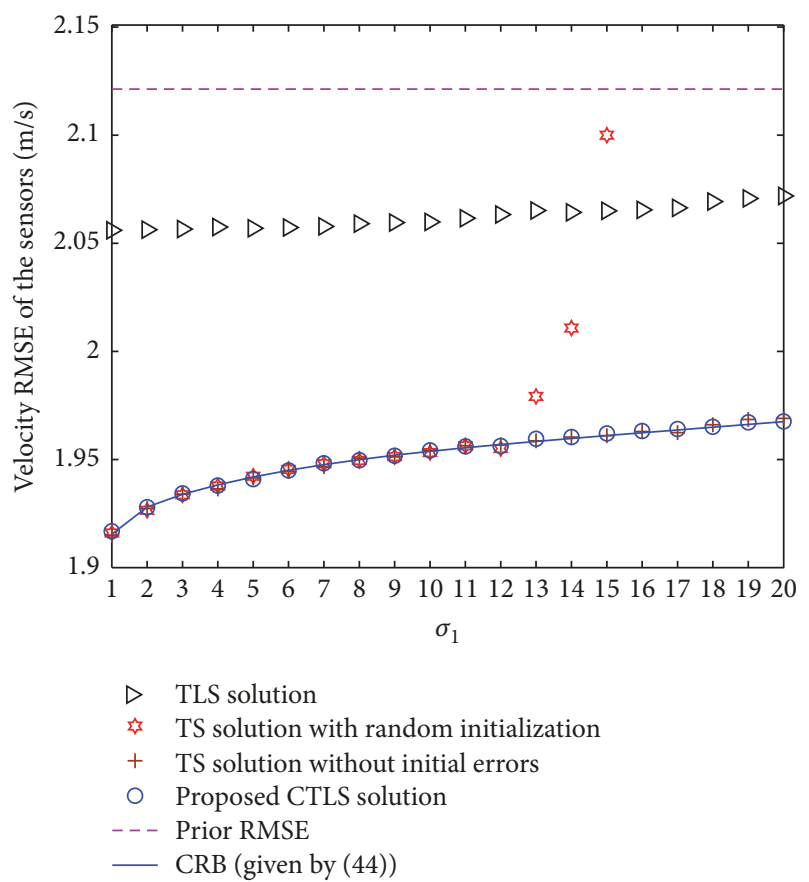

Figure 18: RMSE of the sensor velocity estimate versus $\sigma_{1}$.

We fix $\sigma_{w, p}=20, \sigma_{w, v}=1$ and set $\sigma_{\mathrm{TOA}}=2 \sigma / c, \sigma_{\mathrm{FOA}}=$ $0.02 f_{0} \sigma / c$, where $\sigma$ varies from 1 to 20 . Figures 27 and 28 depict the norm of source position and velocity bias for the three sources versus $\sigma$, respectively.

Figures 27 and 28 demonstrate that the proposed CTLS algorithm can yield very small estimation bias. Moreover, the bias of the CTLS solution is very close to that of the TS

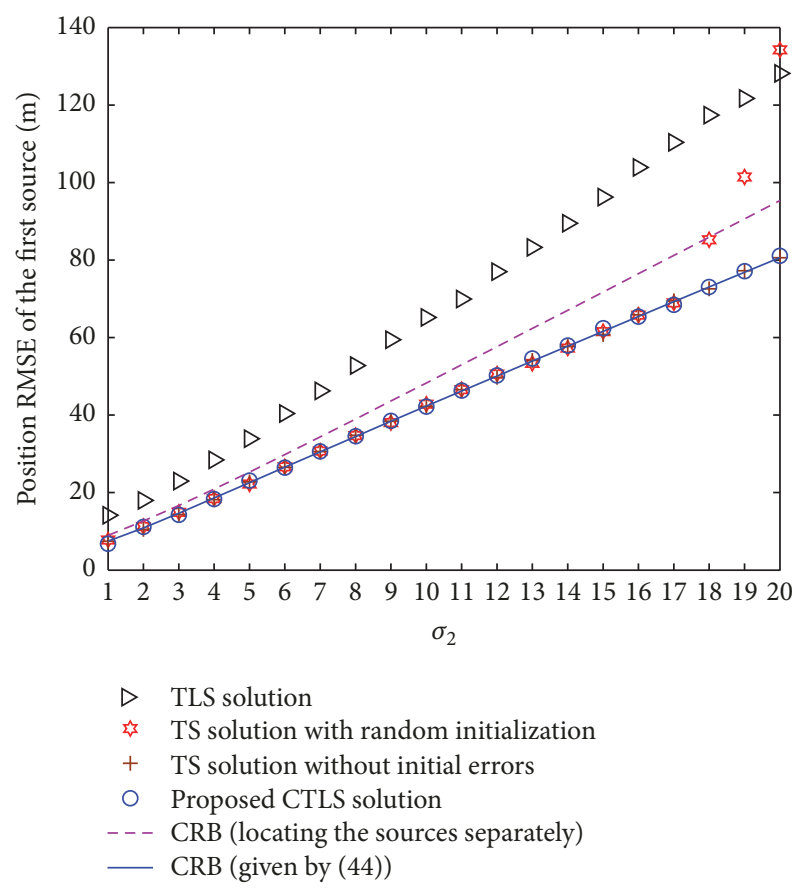

FIGURE 19: RMSE of the estimated position for the first source versus $\sigma_{2}$.

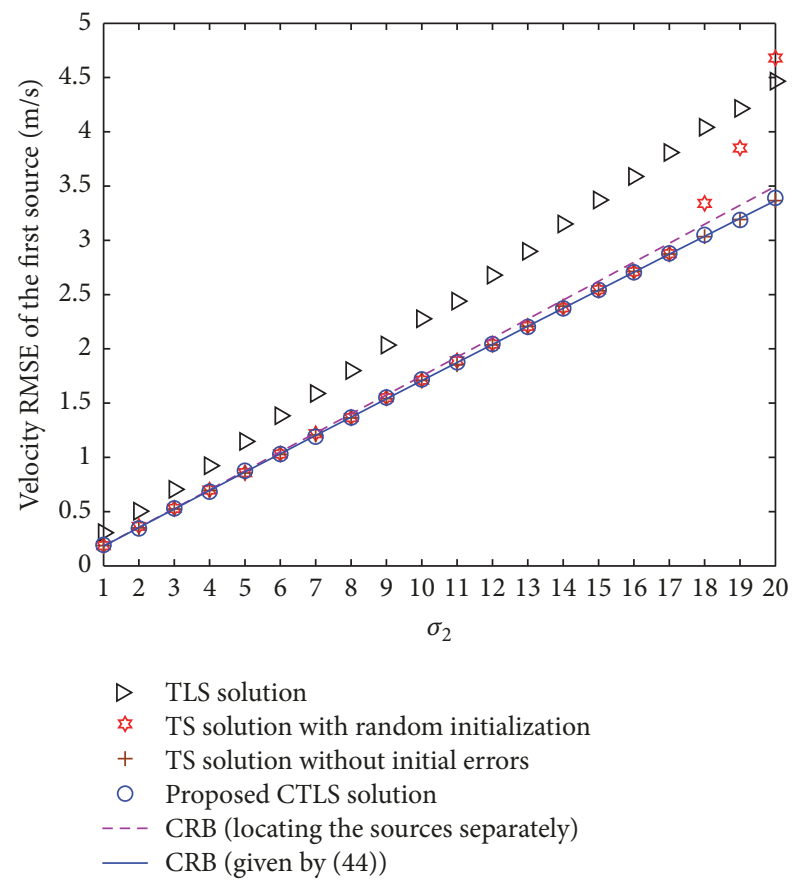

FIGURE 20: RMSE of the estimated velocity for the first source versus $\sigma_{2}$.

algorithm, which is initialized with the true value. But the result of the latter is not displayed in Figures 27 and 28 because it would make the curves rather confusing. Additionally, as shown in Figures 27 and 28, if the TS algorithm is randomly initialized, its estimation bias increases suddenly when the noise level exceeds a certain threshold. This observation is consistent with the conclusion stated above. 


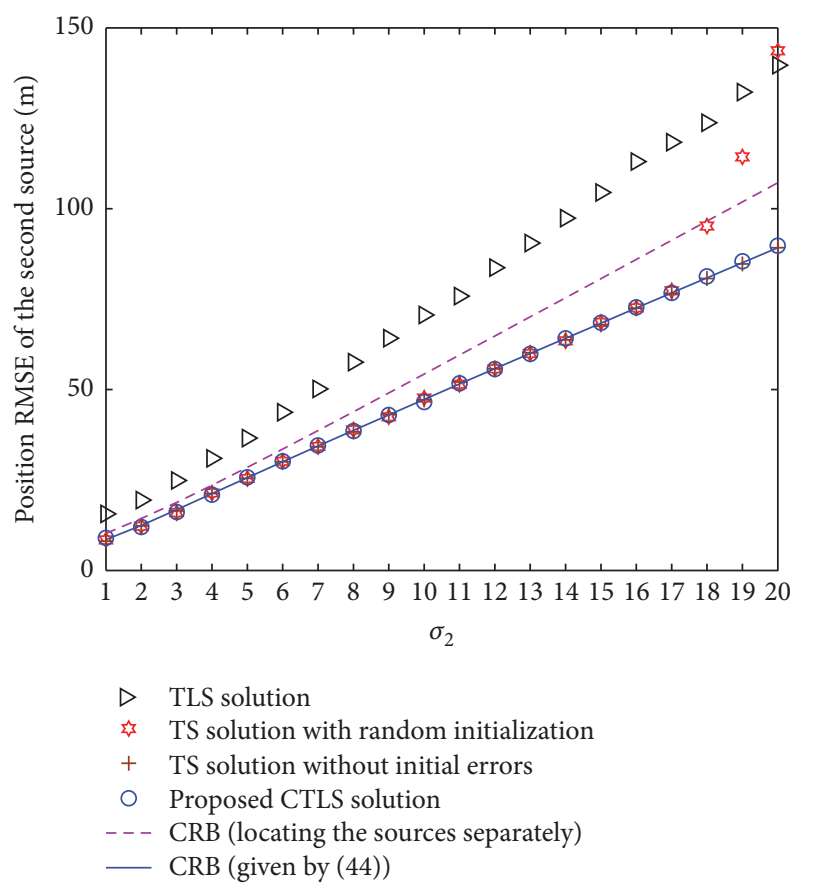

FIGURE 21: RMSE of the estimated position for the second source versus $\sigma_{2}$.

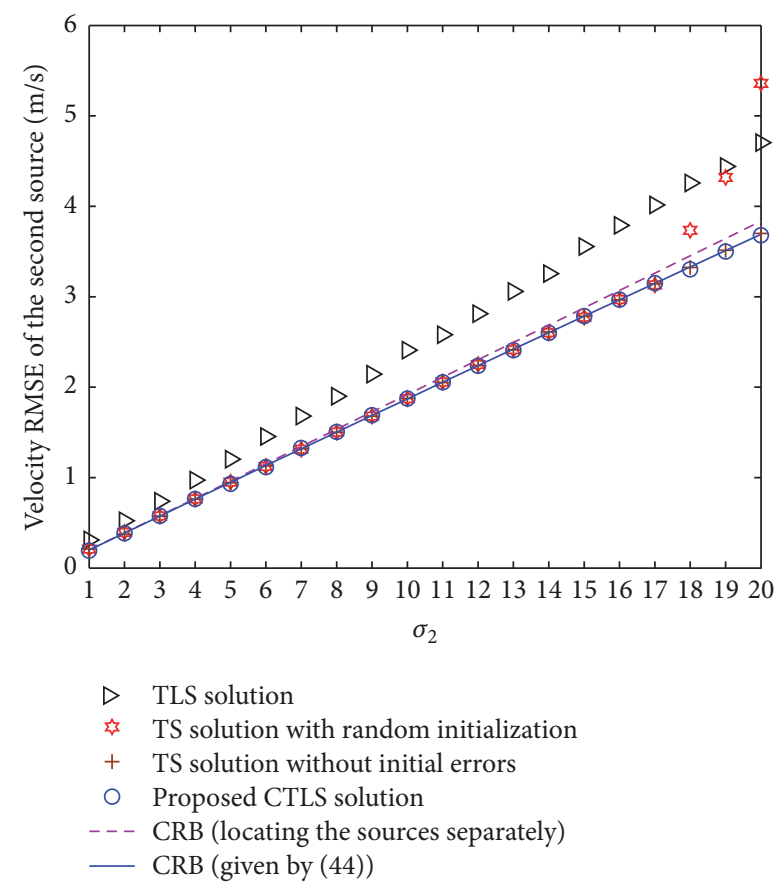

FIGURE 22: RMSE of the estimated velocity for the second source versus $\sigma_{2}$.

\section{Conclusions}

In this paper, we present an efficient CTLS method that can locate multiple disjoint sources and refine the erroneous sensor positions simultaneously. Unlike the conventional localization methods, an important feature of the proposed method is that it establishes a general framework that

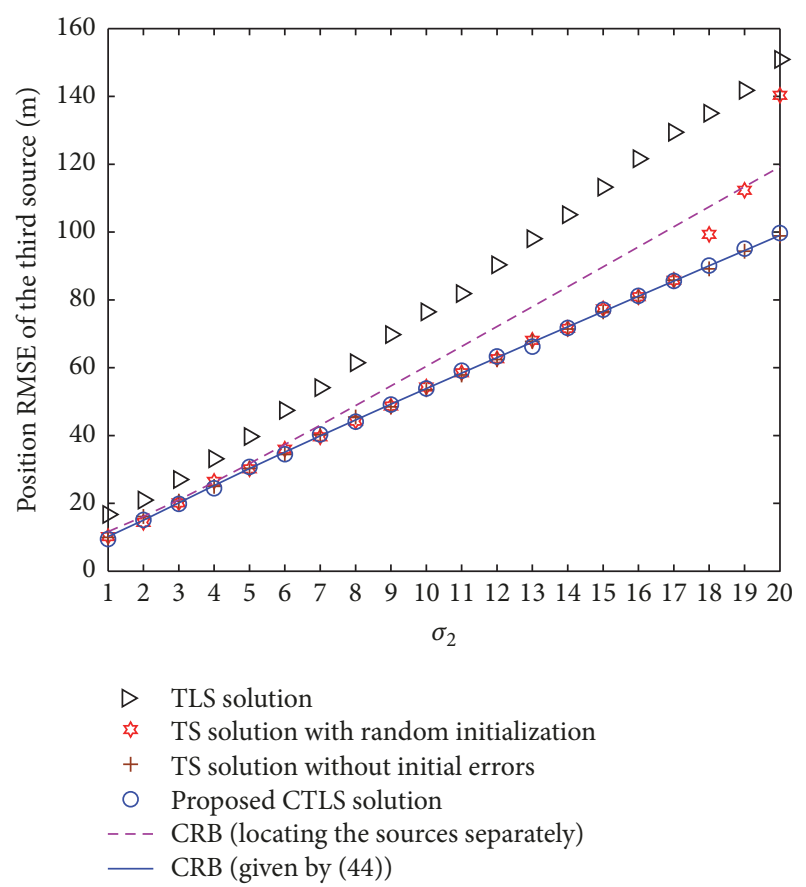

FIGURE 23: RMSE of the estimated velocity for the third source versus $\sigma_{2}$.

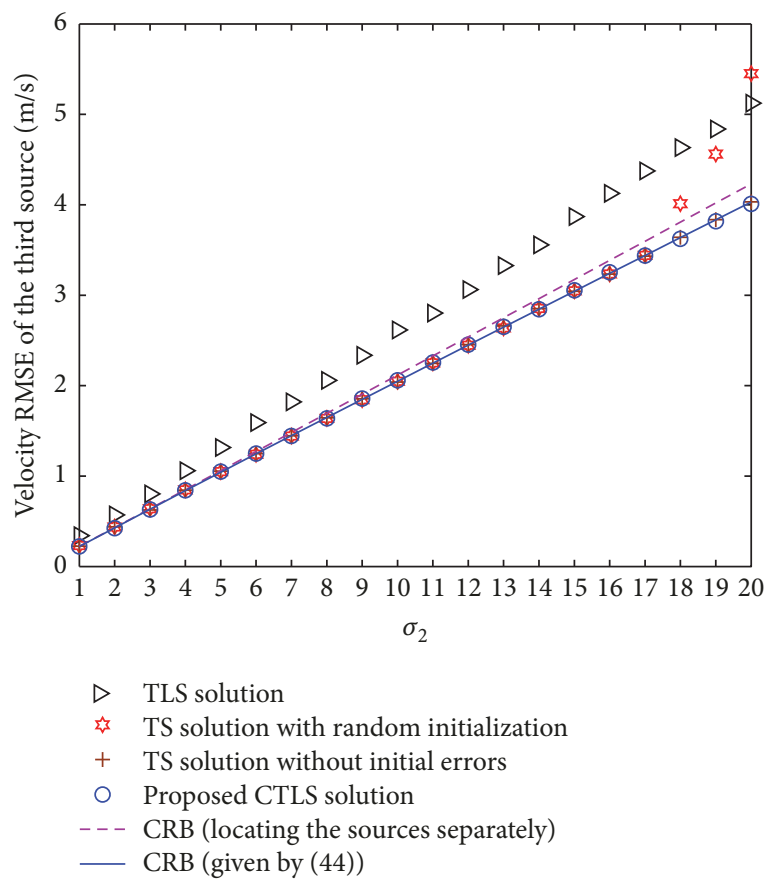

FIGURE 24: RMSE of the estimated velocity for the third source versus $\sigma_{2}$.

is suitable for many different location measurements. A modified CTLS optimization problem is formulated after some algebraic manipulations and the corresponding Newton iterative algorithm is also derived to yield the numerical solution. Besides, by exploiting the first-order perturbation analysis, the exact expression for the covariance matrix of the proposed CTLS estimator is derived under the Gaussian 


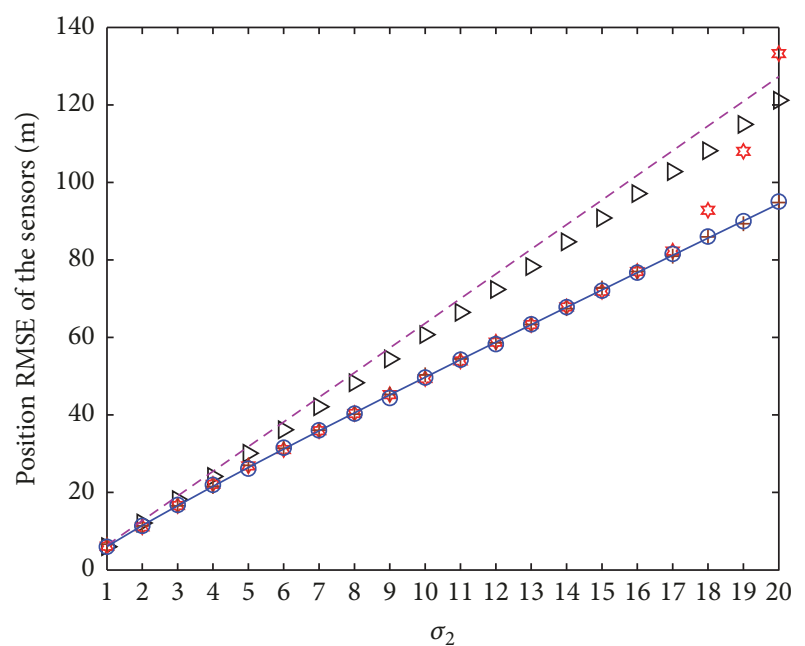

$\triangleright$ TLS solution

TS solution with random initialization

+ TS solution without initial errors

O Proposed CTLS solution

- - Prior RMSE

— CRB (given by (44))

FIGURE 25: RMSE of the sensor position estimate versus $\sigma_{2}$.

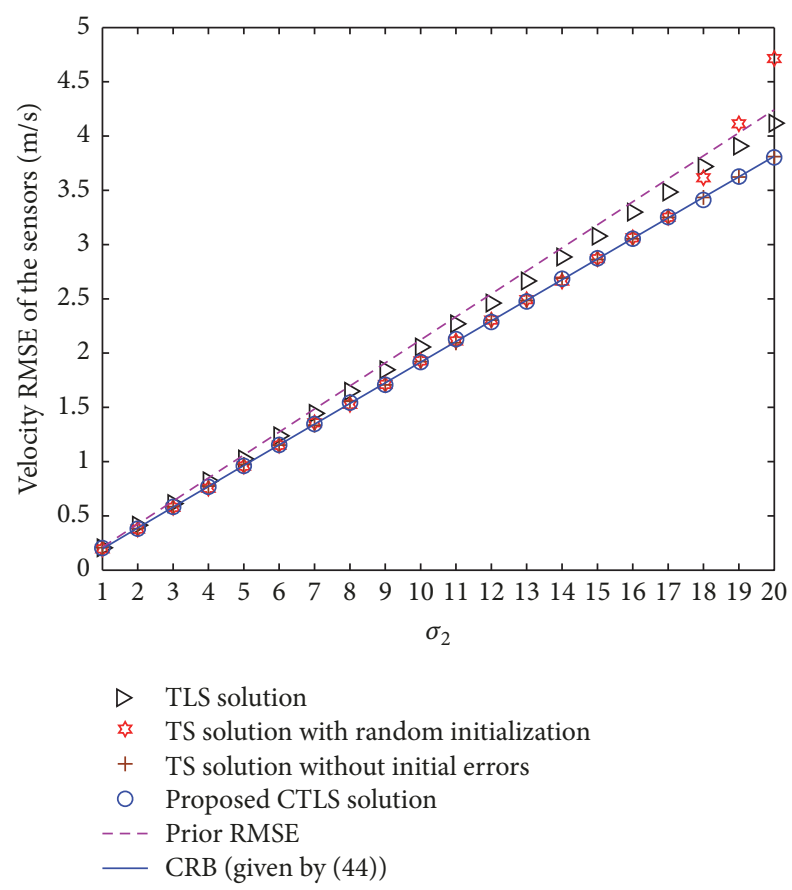

FIGURE 26: RMSE of the sensor velocity estimate versus $\sigma_{2}$.

assumption. The estimation accuracy of the CTLS method is proved to achieve the CRB before the thresholding effect starts to take place. Additionally, two examples are given to explain how to utilize the proposed CTLS method for source localization. One uses the TDOAs/GROAs measurements and the other is based on the TOAs/FOAs parameters. Simulation results verify the good performance of the proposed

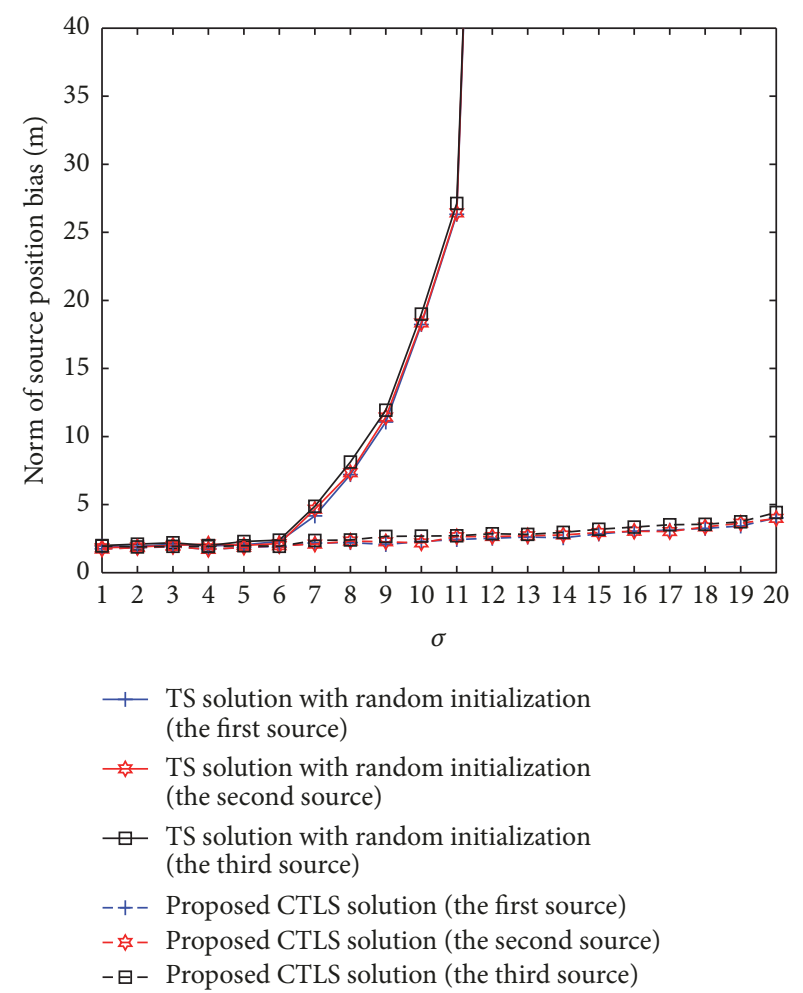

FIGURE 27: Norm of source position bias as a function of $\sigma$.

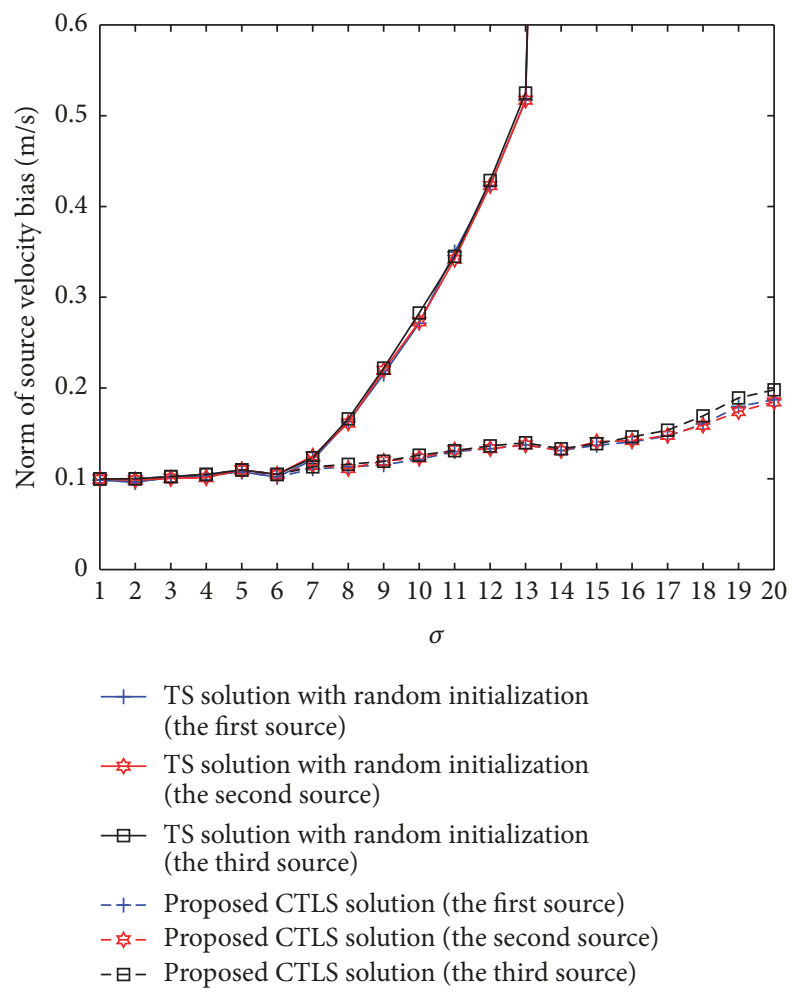

FIGURE 28: Norm of source velocity bias as a function of $\sigma$.

method and also corroborate the performance analysis in this paper. Finally, it is worth emphasizing that the theoretical 
development in this paper is not limited to some specific measurements, and it can be applied to many localization scenarios as long as the measurement equation can be transformed into the pseudo-linear model.

\section{Conflicts of Interest}

The authors declare that there are no conflicts of interest regarding the publication of this paper.

\section{Acknowledgments}

The authors acknowledge support from National Natural Science Foundation of China (Grant no. 61201381, no. 61401513, and no. 61772548), China Postdoctoral Science Foundation (Grant no. 2016M592989), the Self-Topic Foundation of Information Engineering University (Grant no. 2016600701), and the Outstanding Youth Foundation of Information Engineering University (Grant no. 2016603201).

\section{Supplementary Materials}

Supplementary materials contain four appendices, which are called Appendix A, Appendix B, Appendix C, and Appendix D, respectively. (Supplementary Materials)

\section{References}

[1] K. Doğançay, "Bearings-only target localization using total least squares," Signal Processing, vol. 85, no. 9, pp. 1695-1710, 2005.

[2] X. Lu and K. C. Ho, "Taylor-series technique for source localization using AoAs in the presence of sensor location errors," in Proceedings of the 4th IEEE Sensor Array and Multichannel Signal Processing Workshop Proceedings, SAM 2006, pp. 190-194, usa, July 2006.

[3] D. Wang, L. Zhang, and Y. Wu, "Constrained total least squares algorithm for passive location based on bearing-only measurements," Science China Information Sciences, vol. 50, no. 4, pp. 576-586, 2007.

[4] K. W. Cheung, H. C. So, W.-K. Ma, and Y. T. Chan, "Least squares algorithms for time-of-arrival-based mobile location," IEEE Transactions on Signal Processing, vol. 52, no. 4, pp. 11211128, 2004.

[5] Z. Ma and K. C. Ho, "TOA localization in the presence of random sensor position errors," in Proceedings of the 36th IEEE International Conference on Acoustics, Speech, and Signal Processing, ICASSP 2011, pp. 2468-2471, Czech Republic, May 2011.

[6] Y. Zhou, J. Li, and L. Lamont, "Multilateration localization in the presence of anchor location uncertainties," in Proceedings of the IEEE Global Communications Conference (GLOBECOM '12), pp. 309-314, December 2012.

[7] M. Sun, Z. Ma, and K. C. Ho, "Joint source localization and sensor position refinement for sensor networks," in Proceedings of the 2013 38th IEEE International Conference on Acoustics, Speech, and Signal Processing, ICASSP 2013, pp. 4026-4030, Canada, May 2013.

[8] Y. T. Chan and K. C. Ho, "A simple and efficient estimator for hyperbolic location," IEEE Transactions on Signal Processing, vol. 42, no. 8, pp. 1905-1915, 1994.
[9] Y. Huang, J. Benesty, G. W. Elko, and R. M. Mersereau, "Realtime passive source localization: a practical linear-correction least-squares approach," IEEE Transactions on Audio, Speech and Language Processing, vol. 9, no. 8, pp. 943-956, 2001.

[10] H. C. So and S. P. Hui, "Constrained Location Algorithm Using TDOA Measurements," IEICE Transactions on Fundamentals of Electronics, Communications and Computer Sciences, vol. E86A, no. 12, pp. 3291-3293, 2003.

[11] Z. Huang and J. Lu, "Total least squares and equilibration algorithm for range difference location," IEEE Electronics Letters, vol. 40, no. 5, pp. 323-325, 2004.

[12] L. Kovavisaruch and K. C. Ho, "Modified Taylor-series Method for Source and Receiver Localization Using TDOA Measurements with Erroneous Receiver Positions," in Proceedings of the IEEE International Symposium on Circuits and Systems 2005, ISCAS 2005, pp. 2295-2298, jpn, May 2005.

[13] Y. Zhou and L. Lamont, "Constrained linear least squares approach for tdoa localization: A global optimum solution," in Proceedings of the 2008 IEEE International Conference on Acoustics, Speech and Signal Processing, ICASSP, pp. 2577-2580, USA, April 2008.

[14] L. Yang and K. C. Ho, "An approximately efficient TDOA localization algorithm in closed-form for locating multiple disjoint sources with erroneous sensor positions," IEEE Transactions on Signal Processing, vol. 57, no. 12, pp. 4598-4615, 2009.

[15] K. Yang, J. An, X. Bu, and G. Sun, "Constrained total leastsquares location algorithm using time-difference-of-arrival measurements," IEEE Transactions on Vehicular Technology, vol. 59, no. 3, pp. 1558-1562, 2010.

[16] M. Sun, L. Yang, and D. K. C. Ho, "Efficient joint source and sensor localization in closed-form," IEEE Signal Processing Letters, vol. 19, no. 7, pp. 399-402, 2012.

[17] S. Chen, H. He, and H. Yu, "Constrained total least-squares for source location using TDOA measurements in the presence of sensor position errors," Aeronautica et Astronautica Sinica, vol. 34, no. 5, pp. 1165-1173, 2013.

[18] J. Mason, "Algebraic two-satellite TOA/FOA position solution on an ellipsoidal earth," IEEE Transactions on Aerospace and Electronic Systems, vol. 40, no. 3, pp. 1087-1092, 2004.

[19] K. C. Ho and W. Xu, "An accurate algebraic solution for moving source location using TDOA and FDOA measurements," IEEE Transactions on Signal Processing, vol. 52, no. 9, pp. 2453-2463, 2004.

[20] X. N. Lu and K. C. Ho, "Taylor-series technique for moving source localization in the presence of sensor location errors," in Proceedings of the 2006 IEEE International Symposium on Circuits and Systems, pp. 1075-1078, Island of Kos, Greece, 2006.

[21] K. C. Ho, X. Lu, and L. Kovavisaruch, "Source localization using TDOA and FDOA measurements in the presence of receiver location errors: analysis and solution," IEEE Transactions on Signal Processing, vol. 55, no. 2, pp. 684-696, 2007.

[22] S. Xiaoyan, L. Jiandong, H. Pengyu, and P. Jiyong, "Total leastsquares solution of active target localization using TDOA and FDOA measurements in WSN," in Proceedings of the 22nd International Conference on Advanced Information Networking and Applications Workshops/Symposia, AINA 2008, pp. 995999, Japan, March 2008.

[23] H. Wu, W.-M. Su, and H. Gu, "A novel Taylor series method for source and receiver localization using TDOA and FDOA measurements with uncertain receiver positions," in Proceedings of the 6th International Conference on Radar, RADAR 2011, pp. 1037-1040, China, October 2011. 
[24] M. Sun and K. C. Ho, "An asymptotically efficient estimator for TDOA and FDOA positioning of multiple disjoint sources in the presence of sensor location uncertainties," IEEE Transactions on Signal Processing, vol. 59, no. 7, pp. 3434-3440, 2011.

[25] H. G. Yu, G. M. Huang, J. Gao, and B. Liu, "An efficient constrained weighted least squares algorithm for moving source location using TDOA and FDOA measurements," IEEE Transactions on Wireless Communications, vol. 11, no. 1, pp. 44-47, 2012.

[26] H. Yu, G. Huang, and J. Gao, "Constrained total least-squares localisation algorithm using time difference of arrival and frequency difference of arrival measurements with sensor location uncertainties," IET Radar, Sonar \& Navigation, vol. 6, no. 9, pp. 891-899, 2012.

[27] F. Qu and X. Meng, "Comments on 'Constrained total leastsquares localisation algorithm using time difference of arrival and frequency difference of arrival measurements with sensor location uncertainties"' IET Radar, Sonar \& Navigation, vol. 8, no. 6, pp. 692-693, 2014.

[28] B. Hao, Z. Li, J. Si, and L. Guan, "Joint source localisation and sensor refinement using time differences of arrival and frequency differences of arrival," IET Signal Processing, vol. 8, no. 6, pp. 588-600, 2014.

[29] K. C. Ho and M. Sun, "An accurate algebraic closed-form solution for energy-based source localization," IEEE Transactions on Audio, Speech and Language Processing, vol. 15, no. 8, pp. 25422550, 2007.

[30] K. C. Ho and M. Sun, "Passive source localization using time difference of arrival and gain ratios of arrival," IEEE Transactions on Signal Processing, vol. 56, no. 2, pp. 464-477, 2008.

[31] B. Hao, Z. Li, J. Si, W. Yin, and Y. Ren, "Passive multiple disjoint sources localization using TDOAs and GROAs in the presence of sensor location uncertainties," in Proceedings of the 2012 IEEE International Conference on Communications, ICC 2012, pp. 4752, Canada, June 2012.

[32] W. H. Foy, "Position-location solutions by Taylor's series estimation," IEEE Transactions on Aerospace and Electronic Systems, vol. 12, no. 2, pp. 187-194, 1976.

[33] K. W. Cheung, H. C. So, W.-K. Ma, and Y. T. Chan, "A constrained least squares approach to mobile positioning: Algorithms and optimality," EURASIP Journal on Applied Signal Processing, vol. 2006, Article ID 20858, 2006.

[34] X. N. Lu and K. C. Ho, "Analysis of the Degradation in Source Location Accuracy in the Presence of Sensor Location Error," in Proceedings of the 2006 IEEE International Conference on Acoustics Speed and Signal Processing, pp. 14-19, Toulouse, France.

[35] I. Markovsky and S. Van Huffel, "Overview of total least-squares methods," Signal Processing, vol. 87, no. 10, pp. 2283-2302, 2007.

[36] T. J. Abatzoglou, J. M. Mendel, and G. A. Harada, "The constrained total least squares technique and its applications to harmonic superresolution," IEEE Transactions on Signal Processing, vol. 39, no. 5, pp. 1070-1087, 1991.

[37] K. Doğancay, "Relationship between geometric translations and TLS estimation bias in bearings-only target localization," IEEE Transactions on Signal Processing, vol. 56, no. 3, pp. 1005-1017, 2008.

[38] K. C. Ho, "Bias reduction for an explicit solution of source localization using TDOA," IEEE Transactions on Signal Processing, vol. 60, no. 5, pp. 2101-2114, 2012. 


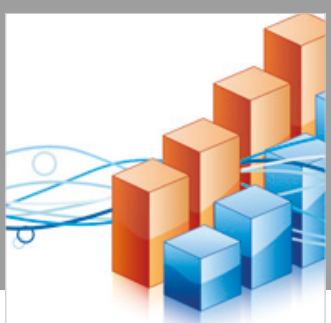

Advances in

Operations Research

\section{-n-m}
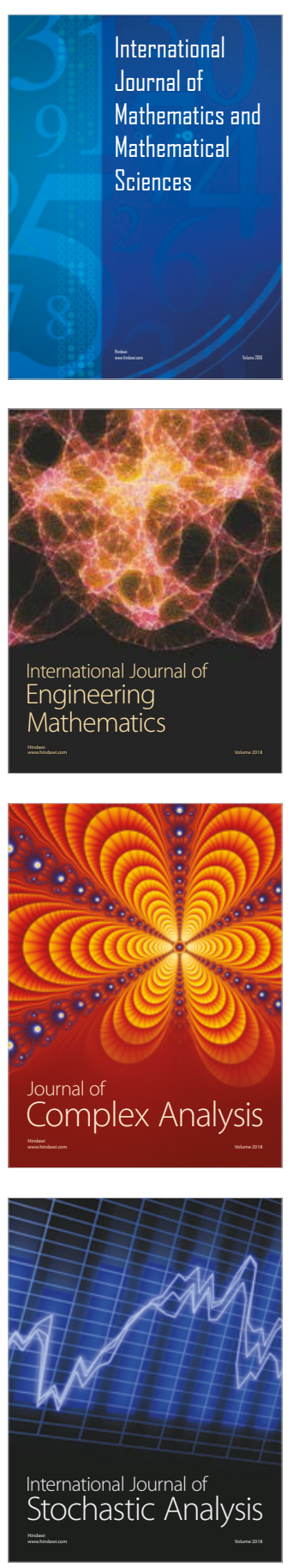
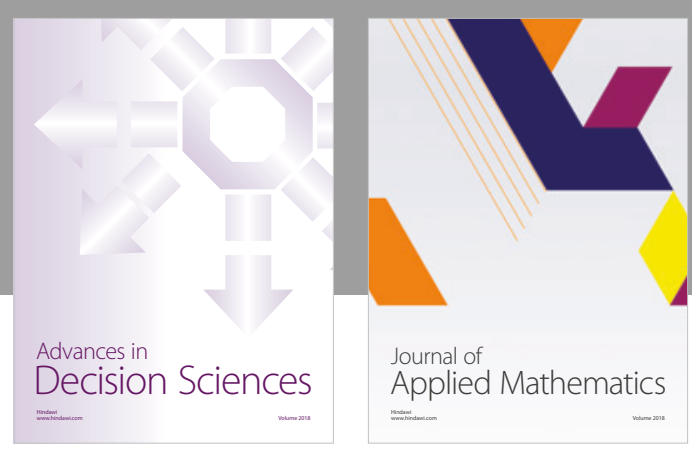

Journal of

Applied Mathematics
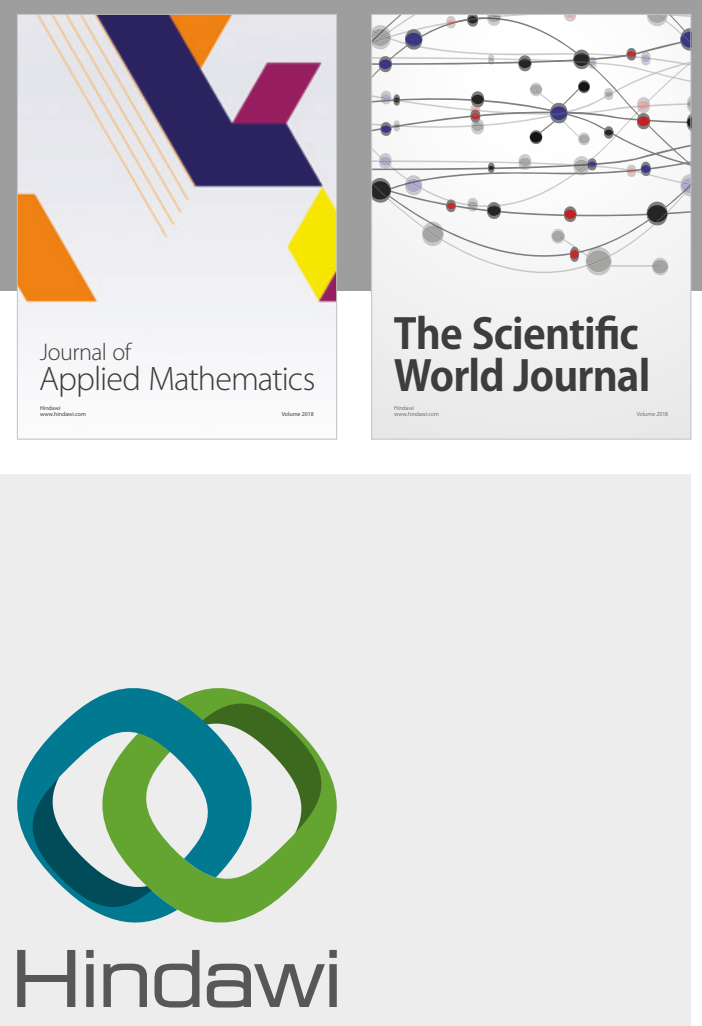

Submit your manuscripts at

www.hindawi.com

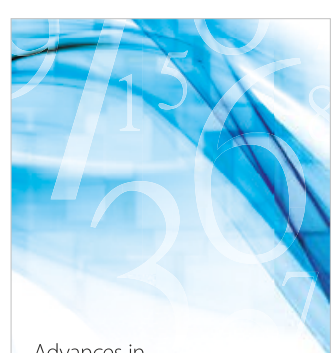

Advances in
Numerical Analysis
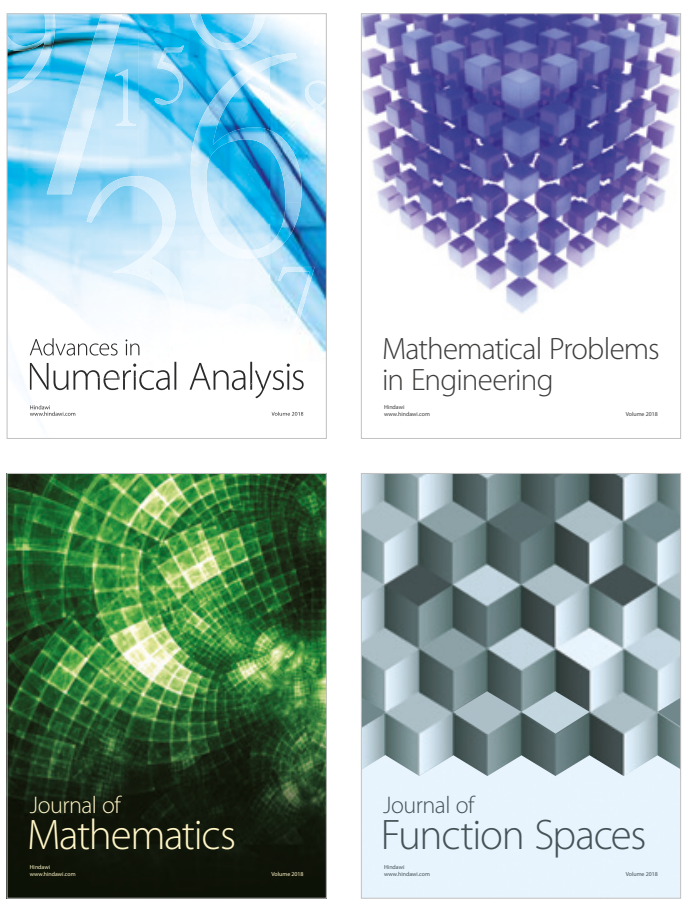

Mathematical Problems in Engineering

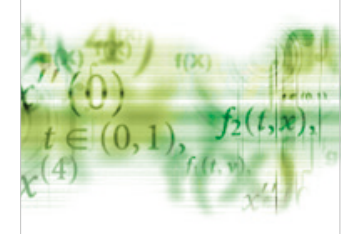

International Journal of

Differential Equations

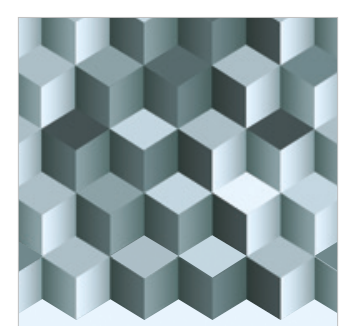

Journal of

Function Spaces

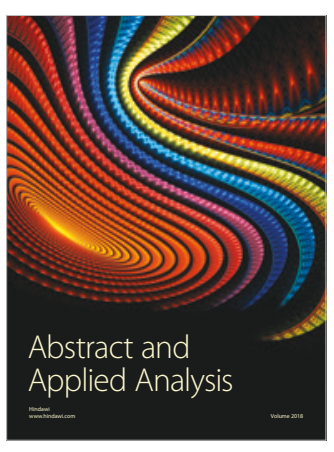

The Scientific

World Journal

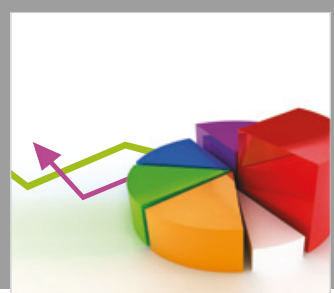

Journal of

Probability and Statistics
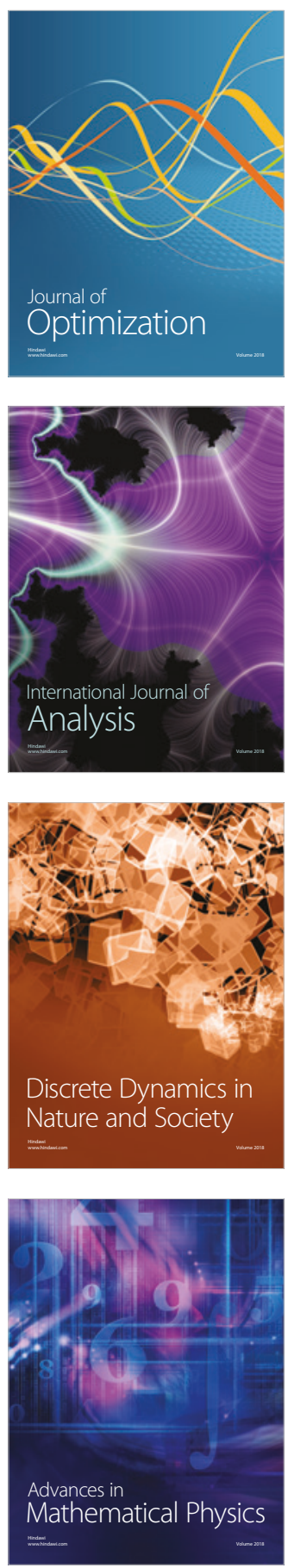